\title{
Automatic Radar Modulation Classification
}

\author{
by
}

Dave Fahlman, B.Eng.

A thesis submitted to the Faculty of Graduate and Postdoctoral Affairs

in partial fulfillment of the requirements for the degree of

\section{Master of Applied Science}

in

\section{Electrical and Computer Engineering}

Ottawa-Carleton Institute for Electrical and Computer Engineering

Department of Electronics

Carleton University

Ottawa, Ontario

June, 2018

Copyright (C) 2018

Dave Fahlman 


\section{Abstract}

Automatic modulation classification is concerned with identifying the modulation present on a radio wave. This can be any type of radar or communication signal. It is employed in fields such as cognitive radio for communications, radar analysis for electronic warfare.

This thesis is dedicated to classifying a variety of modulations used in modern radar. These include unmodulated, various types of frequency modulation, and phase shift keyed waveforms. This task is accomplished through feature extraction and machine learning techniques. The objective is to determine a suitable method applicable for real-time implementation in a complex electronic warfare environment.

Three techniques are proposed: a decision tree combined with Multilayer Perceptron Neural Network, a Multilayer Perceptron Neural Network, and a Convolutional Neural Network. The simulation results show that the decision tree achieves a low classification performance, the Multilayer Perceptron achieves good results in a controlled environment, while the Convolutional Neural Network achieves good generalizable results. The effects of noise, pulse width, and frequency changes are discussed. Each systems latency is also examined. 
This goes out to the twisted transistors and rustic resistors who failed the trials and tribulations of the undergraduate laboratories. May your silicon be forever at peace... 


\section{Acknowledgments}

I would first like to extend many thanks to my supervisor, Dr. Wight, for showing me a great deal about Electrical Engineering. I couldn’t have done it without you! I would also like to acknowledge DTA, specifically Dr. Nijsure and Dr. Roy, for their funding and support of my project. Additionally, I would like to acknowledge the committee members who graciously took time out of their busy schedule to examine my thesis. Thank you!

In addition I would like to thank everybody who helped me! This includes professors, fellow students, and the excellent Department of Electronics staff. This also includes a my friends and my family. My only hope is to bake enough sourdough banana bread for y'all as a proper thank you.

I would also like to thank some important objects, my computer and coffee machine. You were very special to me and I can't imagine writing my thesis without you guys.

Cheers and felicidades! 


\section{Table of Contents}

Abstract $\quad$ ii

Acknowledgments $\quad$ iv

Table of Contents $\quad$ v

List of Tables $\quad$ ix

List of Figures $\quad x$

List of Algorithms xiii

List of Abbreviations $\quad$ xiv

1 Introduction $\quad 1$

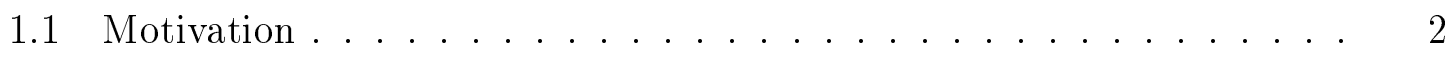

1.2 Objective and Contributions . . . . . . . . . . . 2

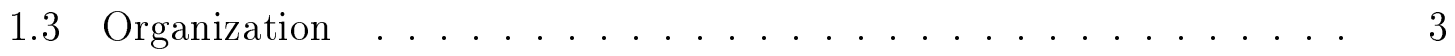

2 Background and Literature Review 4

2.1 Radar Basics ........................ 4

2.2 Type of Modulation . . . . . . . . . . . . . 6

2.2.1 Linear Frequency Modulation ............. 7 
2.2.2 Nonlinear Frequency Modulation . . . . . . . . . . . 8

2.2.3 Barker Code ................... 9

2.2.4 Frank Code ................... 9

2.2.5 Zadoff-Chu Code ................... 11

2.2.6 P Code ........................... 12

2.3 Data Extraction Theory ....................... 14

2.3.1 Likelihood Based Approach _............ 15

2.3.2 Feature Based Approach . . . . . . . . . . . . . 15

2.4 Pattern Recognition Techniques . . . . . . . . . . . . 16

2.4.1 Hierarchical Decision Tree . . . . . . . . . . . 17

2.4.2 Markov Models ................. 17

2.4.3 Artificial Neural Networks . . . . . . . . . . . . . . 18

2.4.4 Convolutional Neural Networks . . . . . . . . . . . . 19

2.5 Literature Review . . . . . . . . . . . . . . . 21

3 Feature and Machine Learning Design 24

3.1 HDT Feature Extraction .................. 25

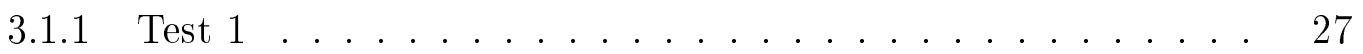

3.1 .2 Test $2 \ldots \ldots \ldots \ldots \ldots \ldots \ldots$

3.1 .3 Test $3 \ldots \ldots \ldots \ldots \ldots \ldots$

3.2 ANN Feature Design ................... 36

3.2.1 Time Frequency Transforms . . . . . . . . . . . . . 36

3.2 .2 Image Analysis and Features . . . . . . . . . . . . 37

4 Simulation Setup $\quad 40$

4.1 Signal Generation . . . . . . . . . . . . . . . 40

4.2 Input and Preprocessing . . . . . . . . . . . . . 41 
4.3 Feature Extraction . . . . . . . . . . . . . . . 42

4.4 Neural Network Training and Parameters . . . . . . . . . . . . . 44

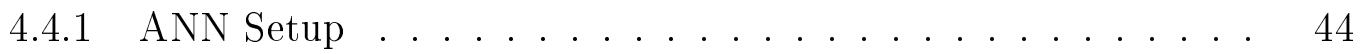

$4.4 .2 \mathrm{CNN} \operatorname{Setup} \ldots \ldots \ldots \ldots$

$\begin{array}{llr}5 & \text { Results } & 48\end{array}$

$5.1 \mathrm{HDT} / \mathrm{ANN}$ Classification . . . . . . . . . . . . 49

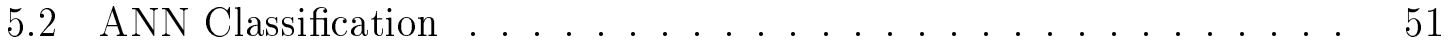

5.3 CNN Classification .................... 56

5.4 Classifier Comparison . . . . . . . . . . . . . . 61

$\begin{array}{llr}6 & \text { Conclusion } & 64\end{array}$

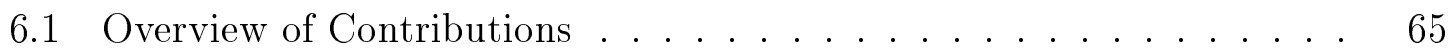

6.2 Future Work . . . . . . . . . . . . . 65

$\begin{array}{ll}\text { List of References } & 67\end{array}$

$\begin{array}{ll}\text { Appendix A MATLAB Generated Waveforms } & 70\end{array}$

A.1 Received Modulations . . . . . . . . . . . . . . 70

A.2 Other Transmitted Modulations . . . . . . . . . . . 77

$\begin{array}{lll}\text { Appendix B MATLAB Code } & 81\end{array}$

$\begin{array}{lll}\text { Appendix C Additional Results } & 94\end{array}$

C.1 ANN Test 1 Confusion Matrices . . . . . . . . . . . . . 94

C.2 ANN Test 2 Confusion Matrices . . . . . . . . . . . . . 98

C.3 ANN Test 3 Confusion Matrices . . . . . . . . . . . . . 101

C.4 CNN Test 1 Confusion Matrices . . . . . . . . . . . . . 104

C.5 CNN Test 2 Confusion Matrices . . . . . . . . . . . 107 
C.6 CNN Test 3 Confusion Matrices . . . . . . . . . . . . . . 110

C.7 Carrier Frequency Confusion Matrices . . . . . . . . . . . . . 113 


\section{List of Tables}

1 Constant Signal Generator Parameters . . . . . . . . . . . . . . 41

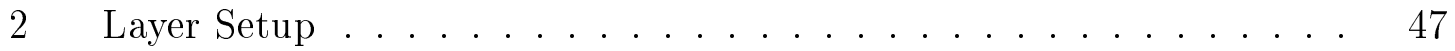

3 Subpulse Parameters . . . . . . . . . . . . . . . 48

4 HDT/ANN Classifier Performance for Test $1 \ldots \ldots \ldots$

$5 \quad$ ANN Classifier Performance for Test $1 \ldots \ldots \ldots \ldots$

6 ANN Classifier Performance for Test $2 \ldots \ldots \ldots \ldots$

7 ANN Classifier Performance for Test $3 \ldots \ldots \ldots \ldots$

8 CNN Classifier Performance for Test $1 \ldots \ldots \ldots$

9 CNN Classifier Performance for Test $2 \ldots \ldots \ldots \ldots$

10 CNN Classifier Performance for Test $3 \ldots \ldots \ldots$

11 Average Latency Per Pulse . . . . . . . . . . . . . . . 62 


\section{List of Figures}

1 Unmodulated pulse . . . . . . . . . . . . . . . . 6

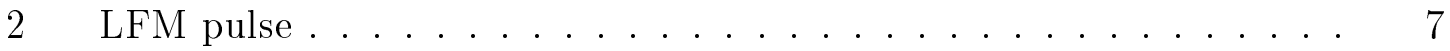

3 Logarithmic NLFM pulse . . . . . . . . . . . . . 8

$4 \quad$ Barker 13 pulse . . . . . . . . . . . . . . 10

$5 \quad$ Frank code pulse $\ldots \ldots \ldots \ldots \ldots \ldots \ldots \ldots$

6 Zadoff-Chu pulse . . . . . . . . . . . . . . . . . . 12

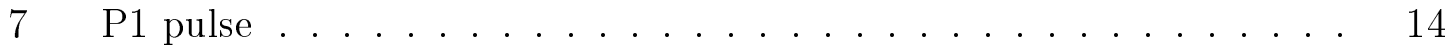

8 Basic concept of a pattern recognition system $\ldots \ldots \ldots \ldots$

9 Example of a two level HDT . . . . . . . . . . . . . 18

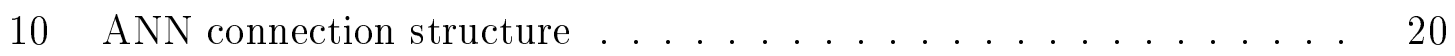

11 Proposed Architecture for First Design . . . . . . . . . . 26

12 Test 1 Flow Chart . . . . . . . . . . . . . . . . 28

13 Instantaneous frequency from Hilbert transform . . . . . . . . . 30

14 Frequency histograms with $\kappa=50 \ldots \ldots \ldots \ldots \ldots$

15 Test 2 Flow Chart $\ldots \ldots \ldots \ldots \ldots \ldots \ldots \ldots \ldots \ldots$

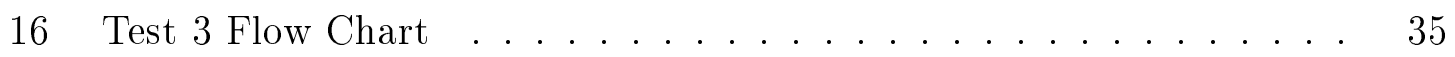

17 P1 and LFM spectrograms using CWD with $\sigma=10 \ldots \ldots \ldots 37$

18 P1 spectrogram using CWD with $\sigma=10 \ldots \ldots \ldots \ldots$

19 Overall ANN Classification Performance . . . . . . . . . . . 51 
20 ANN Performance with Carrier Frequency Variation . . . . . . . . 56

21 Overall CNN Classification Performance . . . . . . . . . 57

22 CNN Performance with Carrier Frequency Variation . . . . . . . . 61

23 Unmodulated received pulse generated in MATLAB . . . . . . . 70

24 LFM received pulse generated in MATLAB . . . . . . . . . . 71

25 NLFM Concave received pulse generated in MATLAB . . . . . . . 71

26 NLFM Convex received pulse generated in MATLAB . . . . . . . 72

27 NLFM logarithmic received pulse generated in MATLAB . . . . . . 72

28 Barker 13 received pulse generated in MATLAB . . . . . . . . 73

29 Zadoff-Chu received pulse generated in MATLAB . . . . . . . 73

30 Frank received pulse generated in MATLAB . . . . . . . . . 74

$31 \quad \mathrm{P} 1$ received pulse generated in MATLAB . . . . . . . . . . 74

$32 \quad$ P2 received pulse generated in MATLAB . . . . . . . . . 75

33 P3 received pulse generated in MATLAB . . . . . . . . 75

34 P4 received pulse generated in MATLAB . . . . . . . . 76

35 Px received pulse generated in MATLAB . . . . . . . . 76

36 Convex NLFM transmitted pulse generated in MATLAB . . . . . . 77

37 Concave NLFM transmitted pulse generated in MATLAB . . . . . 78

38 P2 transmitted pulse generated in MATLAB . . . . . . . . 78

39 P3 transmitted pulse generated in MATLAB . . . . . . . . 79

$40 \quad \mathrm{P} 4$ transmitted pulse generated in MATLAB . . . . . . . . . 79

41 Px transmitted pulse generated in MATLAB . . . . . . . . 80

42 ANN Test 1 Confusion Matrix for $6 \mathrm{~dB} \ldots \ldots . \ldots . \ldots 9$

43 ANN Test 1 Confusion Matrix for $0 \mathrm{~dB} \ldots \ldots . \ldots 96$

44 ANN Test 1 Confusion Matrix for $-6 \mathrm{~dB} \ldots \ldots . \ldots 97$

45 ANN Test 2 Confusion Matrix for $6 \mathrm{~dB} \ldots \ldots . \ldots . \ldots 9$ 
46 ANN Test 2 Confusion Matrix for $0 \mathrm{~dB} \ldots \ldots . \ldots 99$

47 ANN Test 2 Confusion Matrix for $-6 \mathrm{~dB} \ldots \ldots . \ldots 100$

48 ANN Test 3 Confusion Matrix for $6 \mathrm{~dB} \ldots \ldots \ldots 101$

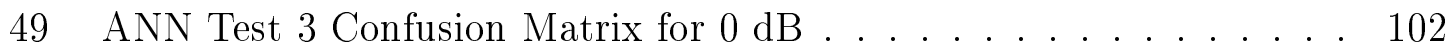

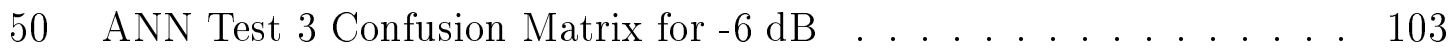

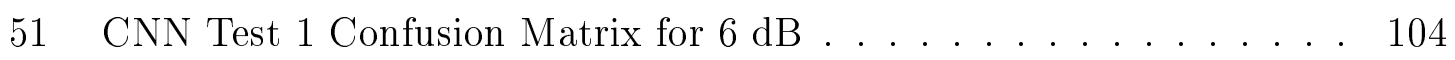

52 CNN Test 1 Confusion Matrix for $0 \mathrm{~dB} \ldots \ldots . \ldots . \ldots 105$

53 CNN Test 1 Confusion Matrix for $-6 \mathrm{~dB} \ldots \ldots \ldots 6$

54 CNN Test 2 Confusion Matrix for $6 \mathrm{~dB} \ldots \ldots \ldots$. . . . . . 107

55 CNN Test 2 Confusion Matrix for $0 \mathrm{~dB} \ldots \ldots . \ldots . \ldots 108$

56 CNN Test 2 Confusion Matrix for $-6 \mathrm{~dB} \ldots \ldots . \ldots . \ldots 109$

57 CNN Test 3 Confusion Matrix for $6 \mathrm{~dB} \ldots \ldots \ldots$

58 CNN Test 3 Confusion Matrix for $0 \mathrm{~dB} \ldots \ldots . \ldots . \ldots 111$

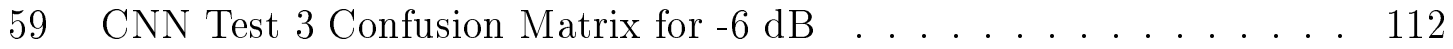

60 ANN Test 4 Confusion Matrix for $6 \mathrm{~dB} \ldots \ldots \ldots \ldots$

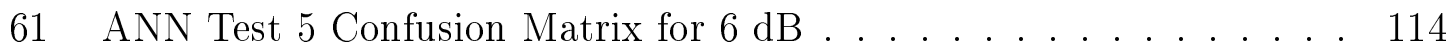

62 CNN Test 4 at Confusion Matrix for $6 \mathrm{~dB} \ldots \ldots . \ldots . \ldots 115$

63 CNN Test 5 Confusion Matrix for $6 \mathrm{~dB} \ldots \ldots \ldots$ 


\section{List of Algorithms}

$1 \quad$ ANNfeatExtPar differences . . . . . . . . . . . . 42

$2 \quad A N N_{-}$Training Header . . . . . . . . . . . . . 46

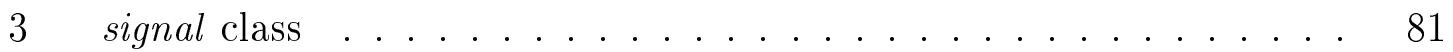

$4 \quad H D T f e a t E x t$ function $\ldots \ldots \ldots \ldots \ldots \ldots \ldots \ldots$

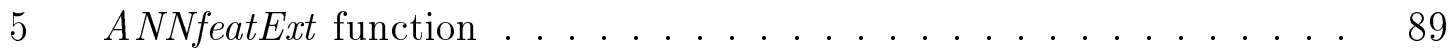

$6 \quad A N N_{-}$Training function $\ldots \ldots \ldots \ldots \ldots \ldots$ 


\title{
List of Abbreviationss
}

\author{
AMC Automatic Modulation Classification \\ ANN Artificial Neural Networks \\ AWGN Additive White Gaussian Noise \\ BPSK Binary Phase Shift Keying \\ BW Bandwidth \\ CNN Convolutional Neural Network \\ CWD Choi-Williams Distribution \\ ELINT Electronic Intelligence \\ EM Electromagnetic \\ EW Electronic Warfare \\ FB Feature Based \\ FM Frequency Modulation \\ FPGA Field Programmable Logic Gate \\ HDT Hierarchical Decision Tree \\ HMM Hidden Markov Model \\ IF Instantaneous Frequency \\ IQ Quadrature Signal \\ LB Likelihood Based \\ LFM Linear Frequency Modulation
}




$\begin{array}{ll}\text { MLP } & \text { Multi Layer Perceptron } \\ \text { MOP } & \text { Modulation on Pulse } \\ \text { NLFM } & \text { Nonlinear Frequency Modulation } \\ \text { PDF } & \text { Probability Density Function } \\ \text { PDW } & \text { Pulse Descriptor Words } \\ \text { PM } & \text { Phase Modulation } \\ \text { PRI } & \text { Pulse Repetition Interval } \\ \text { PSK } & \text { Phase Shift Keying } \\ \text { PW } & \text { Pulse Width } \\ \text { PWVD } & \text { Pseudo Wigner-Ville Distribution } \\ \text { PZM } & \text { Pseudo-Zernike Moment } \\ \text { SNR } & \text { Signal to Noise Ratio } \\ \text { STFT } & \text { Short-Time Fourier Transform } \\ \text { TFA } & \text { Time-Frequency Analysis } \\ \text { WT } & \text { Wavelet Transform }\end{array}$




\section{Chapter 1}

\section{Introduction}

Modern military forces rely extensively on use of the Electromagnetic (EM) spectrum, necessitating the use of Electronic Warfare (EW) to deny an adversary these capabilities. In terms of radar operations, the operational deployment of EW relies on the capture of adversarial radar emissions, called Electronic Intelligence (ELINT). This subfield of EW deals with intercepting and analyzing signals to understand radar system functions and decide upon a reaction. As radars advance and grow more sophisticated, the intelligence gathering capabilities must keep up in order to provide the most functional and complete set of information.

The operational environment that the ELINT systems must operate in is crowded with pulsed radar signals, in the range of 500000 to 1 million per second [1]. Receiving these signals is not difficult as the power density is relatively high. This is due to the power available for a ELINT receiver being inversely proportional to the square of the distance to the receiver, while the power available to the radar is inversely proportional to the fourth power of the range. [2] However this does not mean that the detected signal has a high signal-to-noise ratio (SNR), as the ranges involved are often located beyond the radar horizon. The data signal can be processed in real time

and tagged with Pulse Descriptive Words (PDWs). These are distinct features of the 
EM waveform, such as center frequency, pulse width (PW), time of arrival (ToA) and many others. By tagging PDWs, signal information can be available sooner in combat situations.

\subsection{Motivation}

ELINT information is extremely useful in the event of a conflict. It enables location of anti-aircraft sites, jamming of a defense network, and traffic analysis [2]. As there are more radars in an ELINT environment, data reduction becomes important. If an automated realtime solution can reduce a signal to multiple PDW's, the information can used in a high risk conflict environment. This makes the solution latency one of the driving design factors.

Current solutions can tag aspects such as PW, rise time, bandwidth (BW) and angle of arrival (AoA). The modulation on pulse (MOP) however is more difficult to quantify, yet is a major factor in inferring the purpose of the radar [2]. Many papers have examined this problem, termed Automatic Modulation Classification (AMC). There are many methods to decipher the MOP, with some making use of simple decision trees, while others use varying machine learning algorithms. These solutions can be complicated and impractical for widespread implementation. This work presents a practical, robust solution that can be used on an embedded field platform.

\subsection{Objective and Contributions}

The goal of this thesis is to introduce a realistic solution to the AMC problem. Current state of the art solutions are discussed, along with the challenges inherent to 
the problem. The theory behind radar modulation and the discrimination solution are thoroughly presented. The proposed solution is described and validated through testing and simulation.

This thesis introduces three unique solutions. The first is a combined machine learning approach, utilizing Artificial Neural Networks (ANN) and Hierarchical Design Trees. Secondly, an ANN is designed using feature extraction techniques in both the time and frequency domain. The third is a Convulational Neural Network (CNN), which utilizes Time-Frequency ( $\mathrm{TF}$ ) transformations to produce an image recognition solution. The proposed solutions are tested and simulated, and the results, along with the challenges inherent to the AMC problem, are discussed. Future work is examined, with suggestions including implementation on embedded field platforms. The results have a classification accuracy which is comparable to most systems in literature.

\subsection{Organization}

This thesis is organized in the following manner. Chapter 2 is a literature review which gives both basic background and the state of the art on MOPs discrimination. It also details how this work fits in the current realm of ELINT research. Chapter 3 details the signal processing and feature extraction design. It also specifies the machine learning parameters. Chapter 4 presents the setup of the simulation and implementation. Chapter 5 examines the results and findings from the proposed solution. Finally, Chapter 6 summarizes the work and examines future topics of relevance. 


\section{Chapter 2}

\section{Background and Literature Review}

\section{$2.1 \quad$ Radar Basics}

A radar functions by emitting a pulse, receiving a return, and then accurately determining the round trip time. Knowing the speed of light $c$ and the total time $\delta t$, a distance $R$ can be determined, as per eq. (1).

$$
R=\frac{\delta t c}{2}
$$

Noise has a significant effect in radio wave transmission. It is caused by a variety of sources, including the thermal energy in electronics and nonlinear properties of filters and devices. An important measure of noise is the signal to noise ratio (SNR), which is $N_{0} / E$. This describes the noise power $N_{0}$ on the received signal energy $E$, usually in the units of decibels [1]. Reliable radar operation depends on the SNR, as does ELINT analysis capability.

Another signal distortion is the Doppler frequency shift $f_{d}$. It is caused by the velocity $v_{r}$ of a target relative to the receiver. Noting that $\lambda$ is the radar wavelength, 
the Doppler shift is written as:

$$
f_{d}=\frac{2 v_{r}}{\lambda}
$$

Many other conditions can cause radar signal degradation, including Rayleigh fading and multipath. See [1] for further details.

On the emitted signal, a waveform is obtained by installing frequency or phase modulation on a simple pulse. The choice of modulation directly affects several radar system performance metrics. The most significant impact is on the SNR, range resolution $\delta R$, and Doppler resolution. For example, the modulated pulse length $\tau$ is a limiting factor on how fine a resolution a radar can achieve, as defined in eq. (3). The target can not be distinguished from within the pulse length, leading to a location ambiguity.

$$
\delta R=\frac{\tau c}{2}
$$

A modulation can be both intentional and unintentional. Both types of modulation are distinguishable and are useful features to a ELINT analyst. The intentional modulation is chosen based on the application and technology of a particular radar, and can reveal the radars purpose, as well as aid in specific emitter identification. The unintentional modulation on pulse is created by non-ideal electronics in the radar transmitter, and is a distinguishing feature between unidentified emitters that can otherwise appear as the same radar. This work focuses on the intentional modulation. 


\subsection{Type of Modulation}

The characteristics of an RF signal can be changed in order to transmit information. This is accomplished by combining a carrier wave with a modulation wave, resulting in the desired signal having favourable pulse compression properties. Because the bandwidth of a modulated pulse is proportional to the energy of the transmitted pulse, this enables increased detection range while retaining the range resolution properties of a short pulse. Additionally the radar is less vulnerable to EM interference [1]. An ideal unmodulated pulse is shown below in fig. 1. See Appendix A for examples of received pulses with noise.

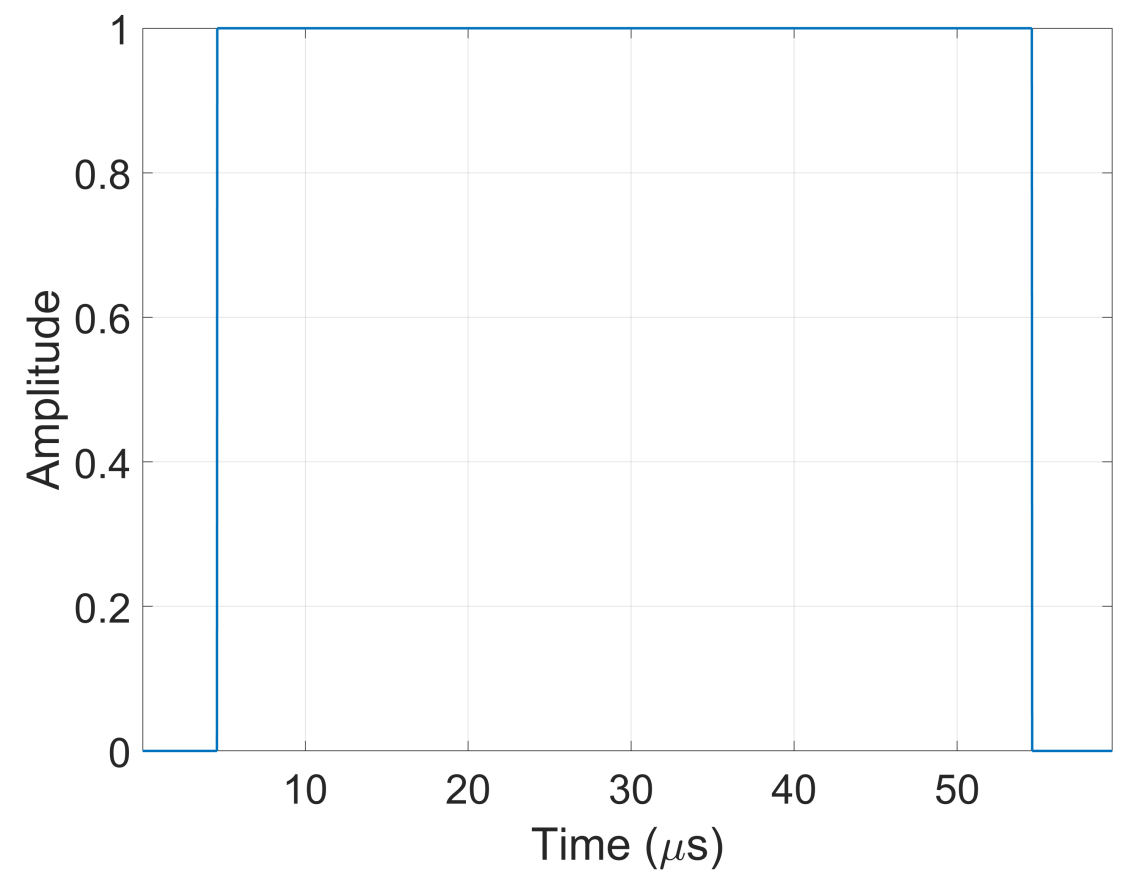

Figure 1: Unmodulated pulse 


\subsubsection{Linear Frequency Modulation}

There are many types of modulation that are applicable for use in radar, the first of which is frequency modulation. Linear frequency modulation (LFM), also known as chirp, has rectangular amplitude $A$ modulation, and is defined by the pulse width, carrier frequency, and chirp slope. The chirp slope $\alpha$, is defined by $\pm B / T$. A positive slope (up chirp) indicates increasing frequency, while a negative (down chirp) indicates decreasing frequency [3]. Using these parameters, the waveform can be defined as:

$$
x(t)=A \operatorname{rect}(t / \tau) \cos \left(\omega t+\pi \alpha t^{2}\right)
$$

where $\omega$ is angular frequency. An example of a LFM signal is shown in fig. 2.

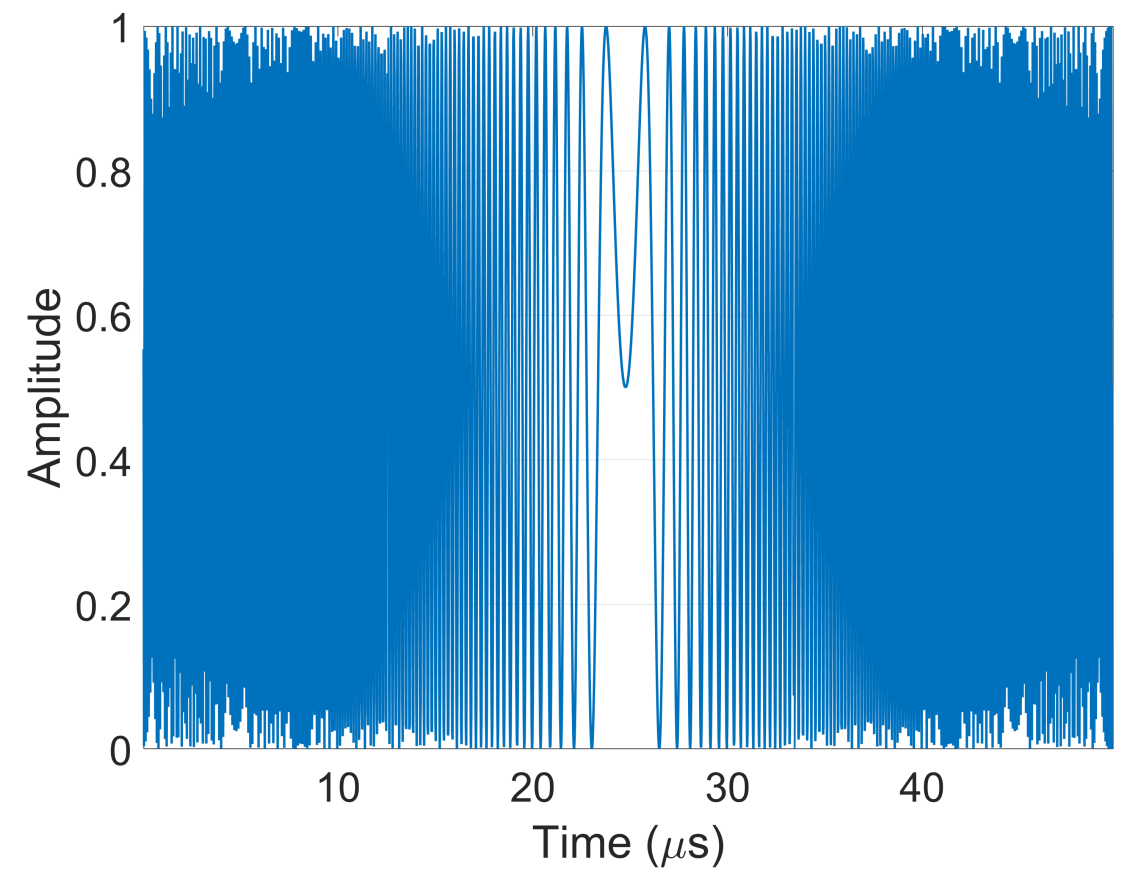

Figure 2: LFM pulse 


\subsubsection{Nonlinear Frequency Modulation}

Another class of waveform similar to LFM is nonlinear frequency modulation (NLFM). However NLFM deviates from the constant chirp rate of frequency change and therefore can shape the pulse to have more desirable characteristics. The general formula for a NLFM wave is seen in eq. (5); the $y(t)$ term refers to any nonlinear mathematical function.

$$
x(t)=A \operatorname{rect}(t / \tau) \cos \left(\omega t+\pi \alpha t^{2}+y(t)\right)
$$

Applications for this modulation scheme are typically tracking systems where the range and Doppler frequency are approximately known, such as detection of rockets and mortars. [4]. An example of a NLFM signal which uses a logarithmic function is shown in fig. 3. See appendix A for examples of concave and convex NLFM.

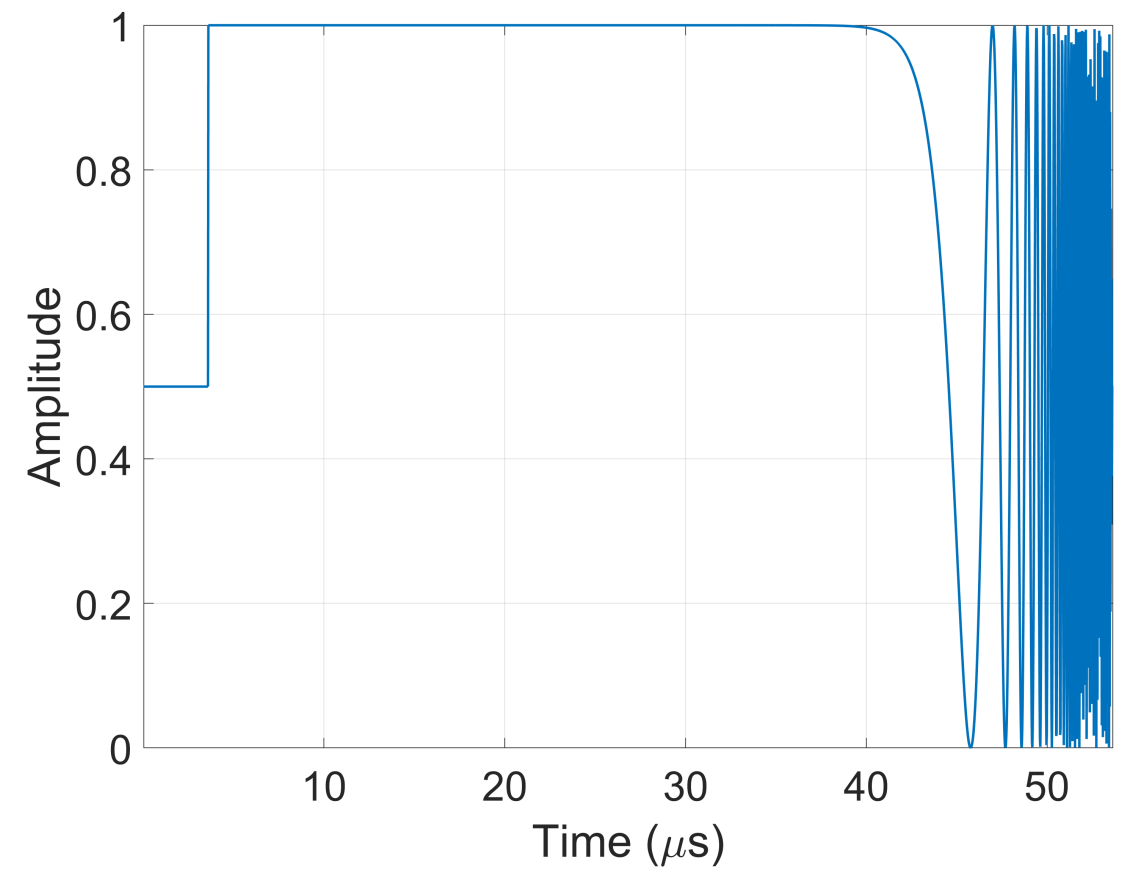

Figure 3: Logarithmic NLFM pulse 


\subsubsection{Barker Code}

Another method of radar modulation is phase shift keying (PSK). This is any waveform that has a constant frequency, and instead relies on switching the absolute phase [4]. The pulse can be split into any number bits $M$ of identical duration, which can then each be coded with a specific value. The values are either biphase, where the bits are binary, or polyphase, which has more than two phase states. Designing phase codes requires evaluating trade offs such as the resulting ambiguity function, frequency spectrum, and ease of implementation [3].

There are many methods of PSK, one of which is the Barker codes. The Barker codes are a specific set of $N$ length biphase bit intervals, which achieve a $M: 1$ ratio of the received signals peak to the highest sidelobe [4]. These sidelobe properties are what makes them useful. The main disadvantage of the Barker codes is that few are available. There are only ten codes known, up to a length of thirteen. Additionally, they are particularly Doppler intolerant, which leads to design constraints [3]. A Barker code where $M=13$ is presented in fig. 4 .

\subsubsection{Frank Code}

Another type of phase coding is the Frank code. This is a polyphase code which has arbitrary values for each bit interval, allowing for lower sidelobes and greater Doppler tolerance. The Frank codes are step approximations of LFM using $M$ number of bits and $M$ number of frequency steps, giving their length as a square of the number of samples $\left(M=L^{2}\right)$. This gives a pulse compression ratio of $M^{2}$, but the fact that the bits must be square is the biggest drawback. The phase code element $s_{m}$ is 


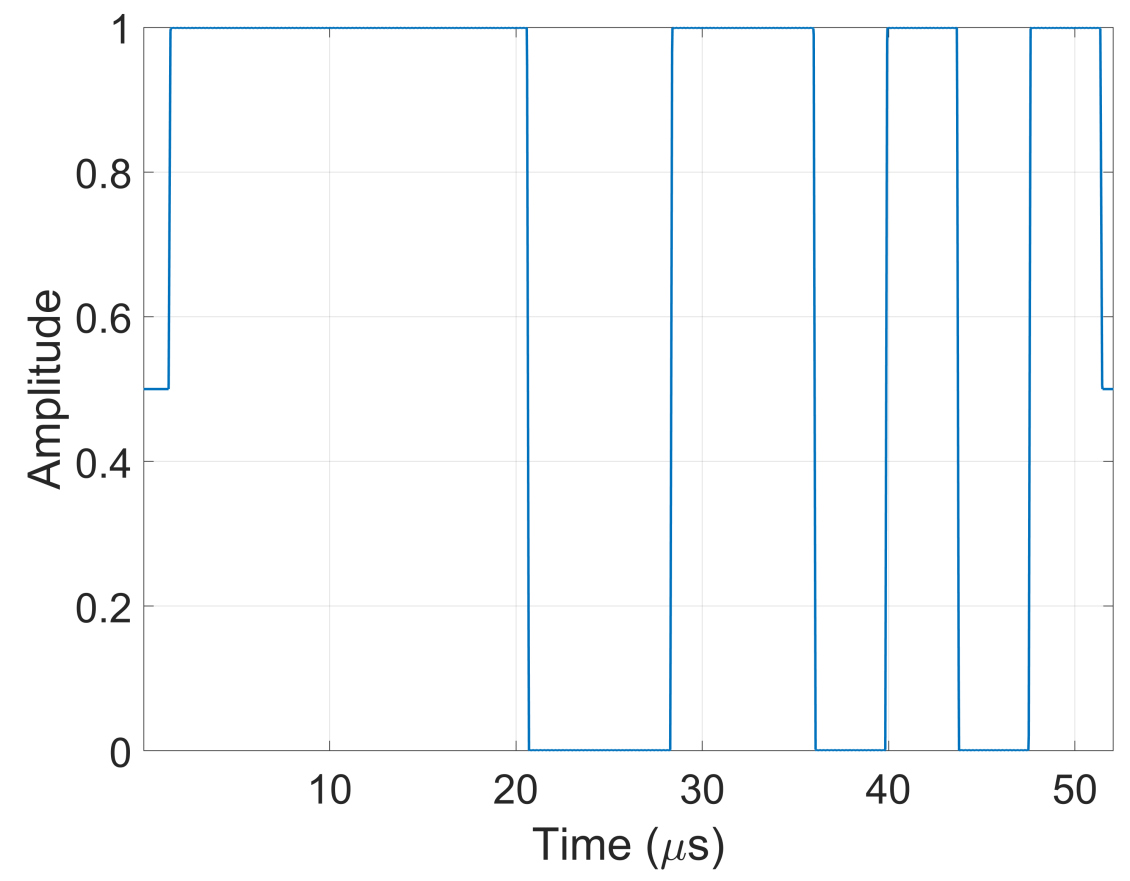

Figure 4: Barker 13 pulse

represented as a phasor, such that:

$$
s_{m}=s_{(n-1) L+k}=e^{j \phi_{n}, k}
$$

The phase $\phi_{n, k}$ of the $n$th sample of the $k$ th frequency is modulated as per eq. (7) [5]. See [3] for the full mathematical derivation.

$$
\phi_{n, k}=\frac{2 \pi}{N}(n-1)(k-1)
$$

$1 \leq n \leq L$, and $1 \leq k \leq L$ are defined as have values that are integers. An ideal Frank code is seen in fig. 5 . 


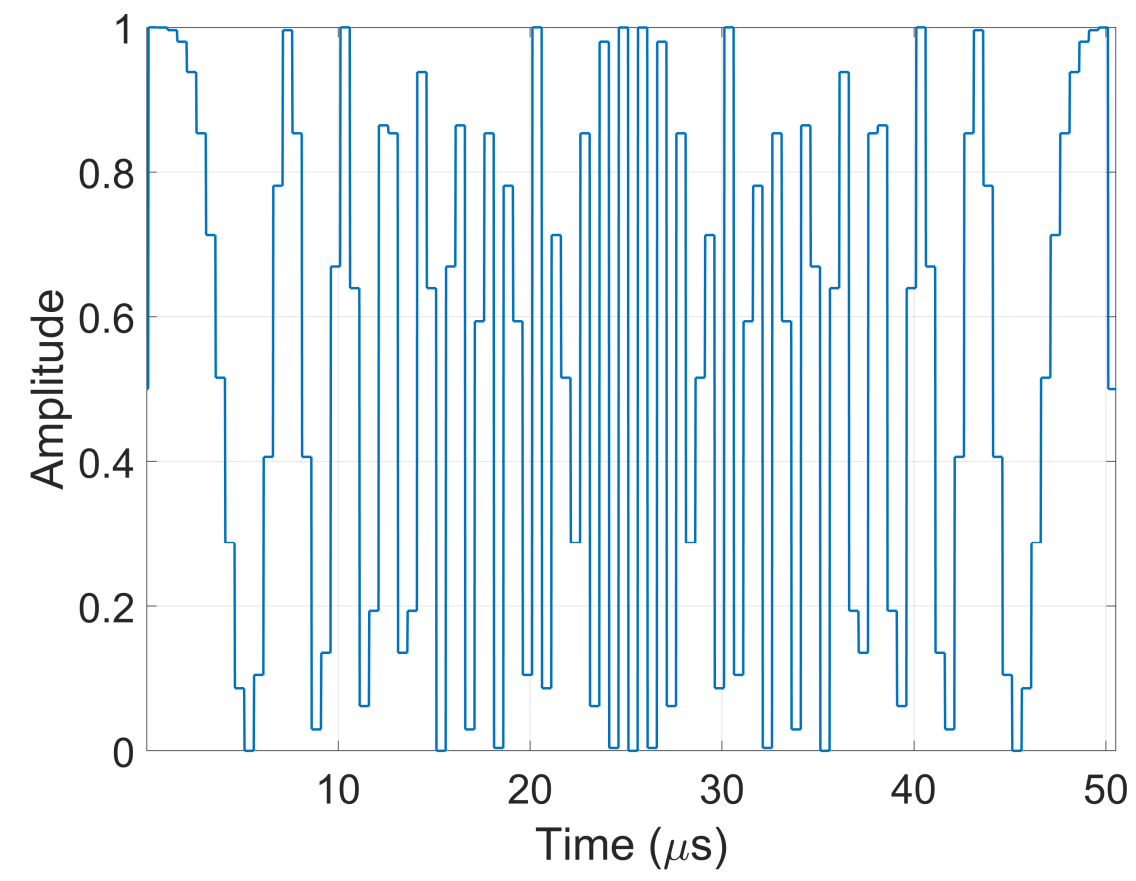

Figure 5: Frank code pulse

\subsubsection{Zadoff-Chu Code}

Another polyphase PSK modulation scheme is the Zadoff-Chu code. The code element $s_{m}$ is represented as a phasor such that:

$$
s_{m}=e^{j \phi_{m}}
$$

These are applicable for any length $M$ and is defined to have a particular phase of $\phi_{m}$. This is represented mathematically by:

$$
\phi_{m}= \begin{cases}\frac{2 \pi r}{M} \frac{(m-1)^{2}}{2}, & \text { for even } M \\ \frac{2 \pi r}{M} \frac{(m-1) m}{2}, & \text { for odd } M\end{cases}
$$




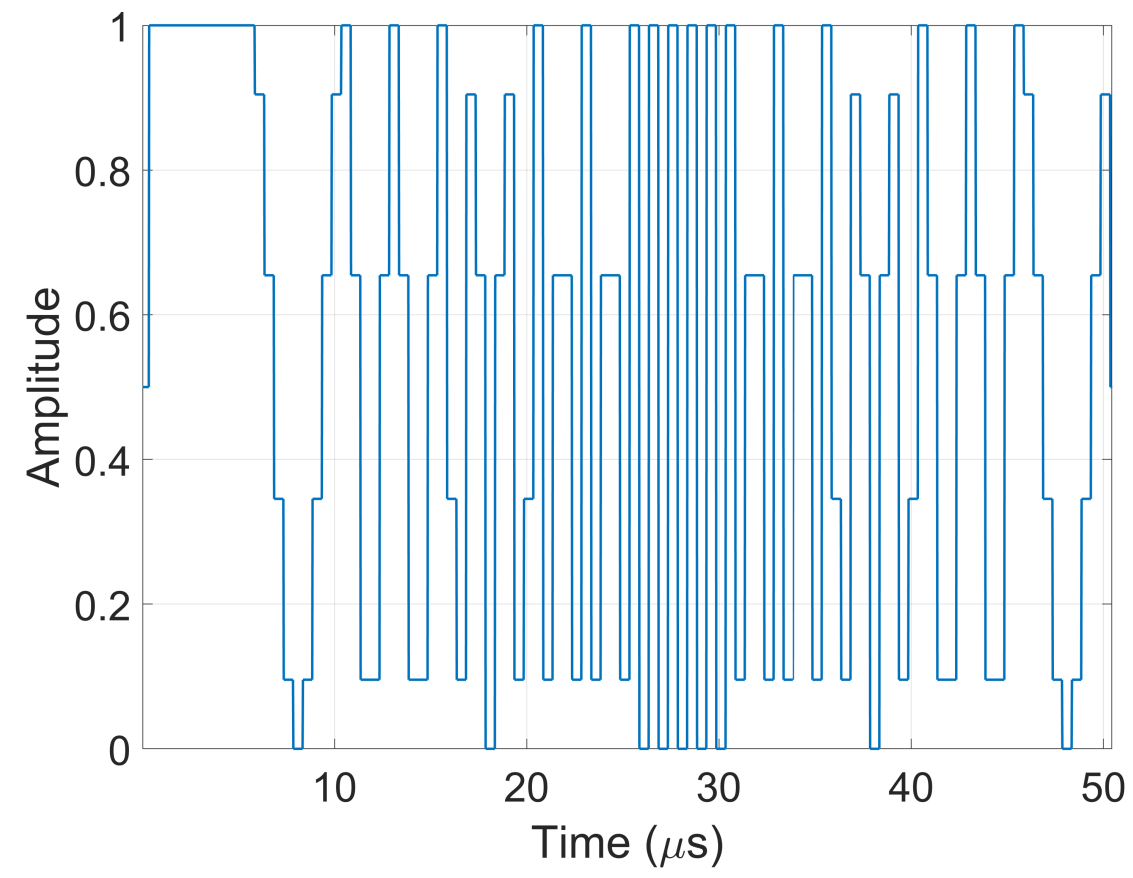

Figure 6: Zadoff-Chu pulse

where $1 \leq m \leq M$ have values that are integers, and $r$ is any integer prime in relation to $M$ [3]. An example of a Zadoff-Chu signal is shown in fig. 6 .

\subsubsection{P Code}

The P1, P2, and Px codes are all derived from the Frank code, and therefore are also step approximations of LFM. Each code element is defined as per eq. (6). However, the DC frequency term is coded in the middle of the pulse, rather than the beginning. These codes are applicable for perfect square length $M$. Of particular interest to this work is defining and comparing each of these codes due to their close similarity. This can lead to ambiguity in classification. The phase modulation scheme for P1 is defined in eq. (11). P1 has the same phase increments as both the P2 and Px codes, except that the starting phase is different. P2 has an even length, with the modulation sequence presented in eq. (12). It has symmetry across each frequency 
row, and symmetry about the center of the pulse [3].

$$
\begin{aligned}
\phi_{n, k} & =-\frac{\pi}{L}(L-(2 k-1))((k-1) L+(n-1)) \\
\phi_{n, k} & =\left(\frac{\pi(L-1)}{2 L}-\frac{\pi(n-1)}{L}\right)(L+1-2 k)
\end{aligned}
$$

The Px code exists for all square valued bit sequences, giving a length of $M=$ $L^{2}$. These codes were defined relatively recently, and outperform all other sequences defined here in terms of sidelobe ratios [6]. The phase sequence is defined as:

$$
\phi_{n, k}= \begin{cases}\frac{2 \pi}{L}\left(\frac{L+1)}{2}-k\right)\left(\frac{L+1)}{2}-n\right), & \text { for even } L \\ \frac{2 \pi}{L}\left(\frac{L}{2}-k\right)\left(\frac{L+1)}{2}-n\right), & \text { for odd } L\end{cases}
$$

where $1 \leq n \leq L$, and $1 \leq k \leq L$ are defined as have values that are integers.

The P3 and P4 codes are specific shifted and decimated versions of the ZadoffChu Code. Each phase element is defined as per eq. (8). Unsurprisingly, they are also applicable for any length $M$. These codes have been shown to be very Doppler tolerant. The phase sequences for P3 and P4 are defined in eq. (15) and eq. (16) respectively. It is noted that the $\mathrm{P} 4$ code is distinguished from the P3 by having the largest phase changes at the end, rather than in the middle. This makes $\mathrm{P} 4$ is more bandwidth tolerant. The phase sequences are defined as:

$$
\begin{aligned}
\phi_{m} & =\frac{2 \pi}{M} \frac{(m-1)^{2}}{2} \\
\phi_{m} & =\frac{2 \pi}{M}(m-1)\left(\frac{m-1-M}{2}\right)
\end{aligned}
$$

where $1 \leq m \leq M$ are defined as integer values. For further derivations and discussion related to the $\mathrm{P}$ codes, refer to [3]. Figure 7 gives an example of P1 modulation, and 
figs. 38 to 41 in appendix A for the P2, P3, P4 and Px waveforms.

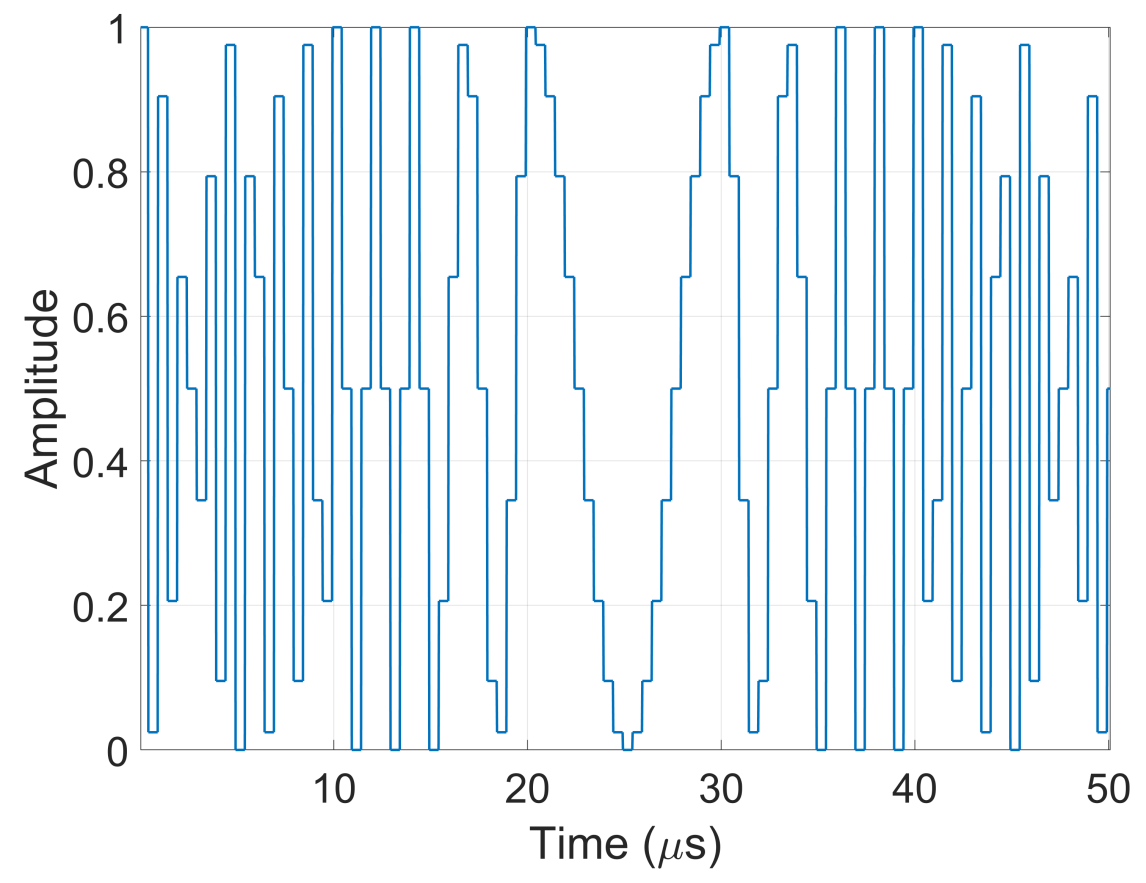

Figure 7: P1 pulse

While there are many other modulations that are used in modern radar, these thirteen types provide a good sample of what could be encountered. Furthermore, it is shown that the classification approach can be generalized for any signal.

\subsection{Data Extraction Theory}

AMC is defined as any method which, given received data samples, can automatically determine the transmission modulation. There are many applications in all types of ELINT, from communication systems to radar. This includes a wide variety of areas including spectrum management, cognitive radio, and electronic warfare [7]. Given the wide scale application, it is unsurprising that there are a large number of techniques that have been developed for this purpose. In general, they can be placed 
in one of two categories: likelihood based (LB) or feature based (FB).

\subsubsection{Likelihood Based Approach}

LB algorithms offer the optimal solution: they minimize the probability of false classification. This is done on the premise that the probability density function (PDF) contains all needed information about a specific waveform. A modulation is classified by computing a likelihood ratio from the PDF, and comparing this to a decision threshold [8]. The issue lies in these solutions being computationally complex. In a real time classification environment there are many pulses and many emitters to analyze. Other techniques can be used to find the PRI, enabling separation of a specific emitter. This allows some leeway with modulation classification, as a few false classifications can be ignored if the majority are correct [9]. Another factor in extracting the data is algorithm robustness. LB methods are not inherently robust, and are corrupted by effects such as noise, timing errors, and phase offsets. Considering this, and the need for real-time operation, a less complex feature based method can be used.

\subsubsection{Feature Based Approach}

FB approach utilizes representative features to highlight differences in each signal, allowing them to be classified. Features are commonly taken from the time domain, signal transformations, zero crossings and statistics. The FB AMC method often takes a hierarchical approach, first sorting the modulation into classes, and then identifying the specific type [8]. The decision-making methods vary greatly, and include PDF based, Euclidean distance, and artificial intelligence classifiers. Even with suboptimal performance, FB algorithms are generally preferred due to low complexity 
and satisfactory performance.

\subsection{Pattern Recognition Techniques}

The goal of pattern recognition is to classify inputs into a fixed number of discrete classes [10]. This has many applications including machine vision, character recognition, and signal discrimination. In the context of this thesis, pattern recognition is used to sort and label radar signal data based on the MOP. In order to classify a signal's modulation, information must be extracted that provides a discrete and unique profile. This information, called the features, are then put through an algorithm that separates the input into classes based on the features. Figure 8 demonstrates this process.

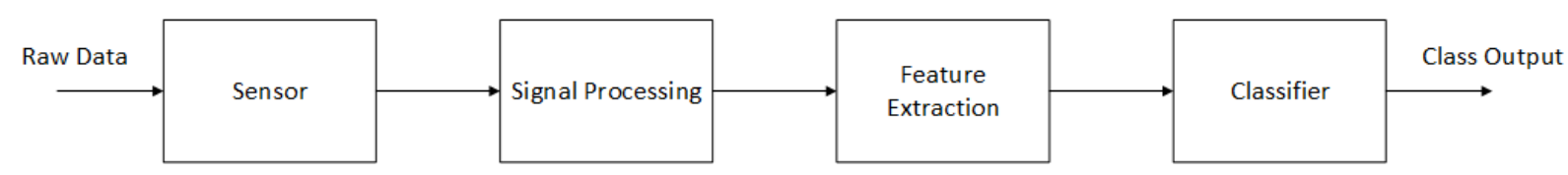

Figure 8: Basic concept of a pattern recognition system

Machine learning algorithms are often used for pattern recognition, due to their ability to find and correlate information that humans have difficulty seeing. One of the biggest challenges in machine learning is obtaining or creating training data. There are two schools of learning: unsupervised and supervised. Unsupervised data learning is also known as clustering [11]. The goal is to determine the underlying similarities and form groups from this information. This works well when there are no target outputs. Supervised learning develops a model and determines the decision regions offline using labeled training data. This information is then used to compare and determine the most likely classification. This allows a large number of potential features and classes, and is used when large amounts of pre-labeled data is available. A 
simple method for clustering is presented first, with some supervised machine learning models following.

\subsubsection{Hierarchical Decision Tree}

The simplest nonparametric method to classify patterns is a hierarchical decision tree (HDT). To find a solution the tree makes a sequential decision, based on one or more thresholds. These sequences step down the hierarchy one at a time, requiring one or more decisions before a classification can be reached. The advantage of this method is the simplicity and ease of implementation, making it useful for coarse clustering in a real time scenario. However, unless care is used to use noise invariant statistics, false classifications can arise due to differing signal environments. Figure 9 presents an example of a two level HDT. This tree is capable of classifying 3 different patterns, distinguished by two decision thresholds. See [12] for more detailed information on HDTs.

\subsubsection{Markov Models}

Markov models are based on the Markov chain, which is a way of describing a progression of events through state sequences. Essentially, the next state is only considered to be dependent on the current state. Any process satisfies the Markov property if predictions for the future of the process are the same regardless of knowing the state history. This implies that Markov Models are memoryless.

In a Hidden Markov Model (HMM) the states are not known, and instead observations are made. Each observation has a probability distribution related to each state. Therefore, the sequence of observation gives some information about the sequence of states. This allows the modeling of uncertainty and imperfections. For example, in a 


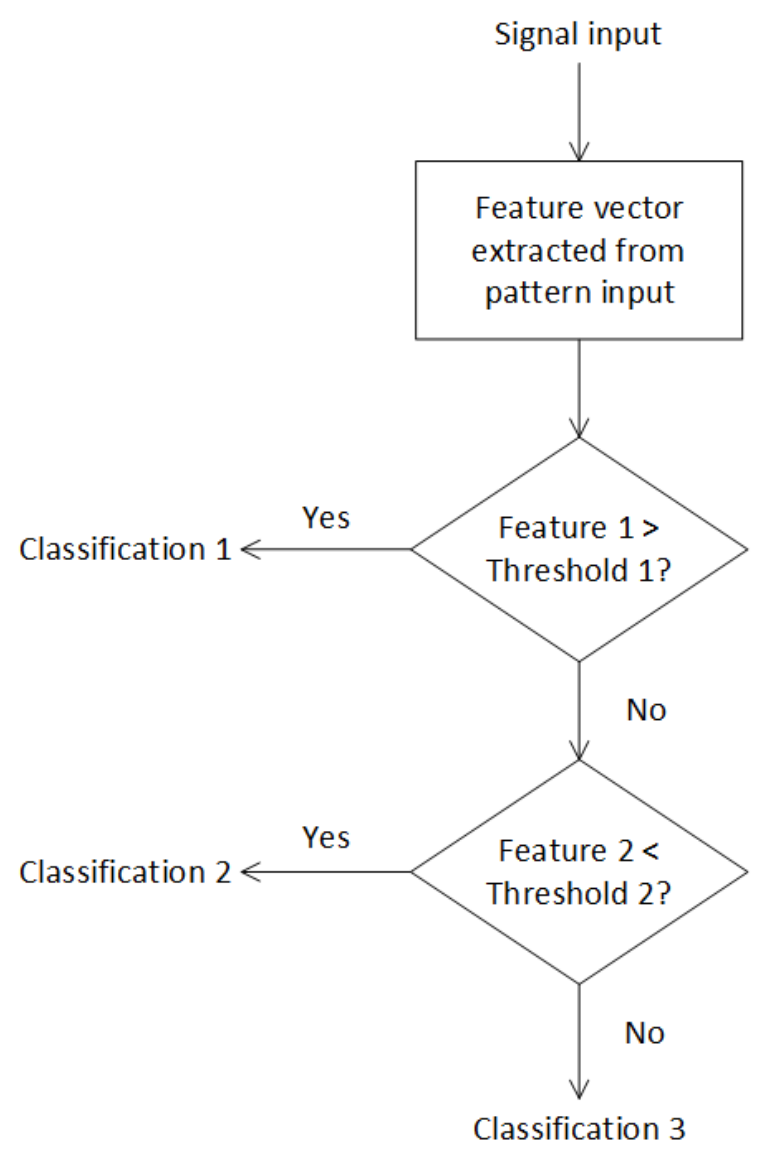

Figure 9: Example of a two level HDT

radar signal the ideal waveform would be the states. The received signal with noise and other distortions would be the observation. HMM and variants are extensively used in fields such as gesture recognition, signal analysis and speech synthesis.

\subsubsection{Artificial Neural Networks}

An Artificial Neural Network (ANN) is a system of machine learning that is inspired by the workings of the biological brain [11]. The basic idea is a web of interconnected neurons that, when weighted correctly, can estimate a non-linear relationship between the input and output of a system. ANNs can find trends and patterns in data that may be difficult to extract using conventional techniques. One of the advantages 
to using a ANN is that it can construct nonlinear decision thresholds between different classes in a non-parametric fashion. This offers a practical way of performing complex pattern recognition. However, in order to extract these relations they must first be trained on labeled data. This is followed by a testing stage, where known inputs are presented to the trained network and the output analyzed to determine the performance of a classifier. If this is found to be satisfactory, the ANN is ready to classify unknown inputs and produce useful results.

ANNs have two main connection structures: single or multilayer. An overview of the basic connection structure is presented in fig. 10. In the former, inputs $\hat{x}$ are connected to each other via one layer of neurons. This layer can be any number of neurons, defined in the reference diagram as $1 \leq j \leq J$. Each connection to this layer is multiplied by weightings $\hat{w}_{x, j}$ decided by the training. This layer then feeds forward to give the final output, feature vector $\hat{y}$. This however, has many limitations and cannot accurately describe a complex system [13]. The discovery of back propagation enabled the training of multilayer feed forward ANN [14]. The multilayer ANN has layers defined by a range of integers $1 \leq m \leq M$, with each layer having its own range of neurons and connections. A specific type, the Multilayer Perceptron (MLP), is an ANN architecture which has proved to be particularly adept in solving complex pattern recognition problems. For an overview of this and other popular types of networks such as Radial Basis Function networks see [12], [13] or [14].

\subsubsection{Convolutional Neural Networks}

The Convolution Neural Network (CNN) is a type of Neural Network with many non-linear neurons stacked on top of each other. These networks are deep, meaning they have a relatively large number of layers (approximately 6 to 10) [15]. Previously 


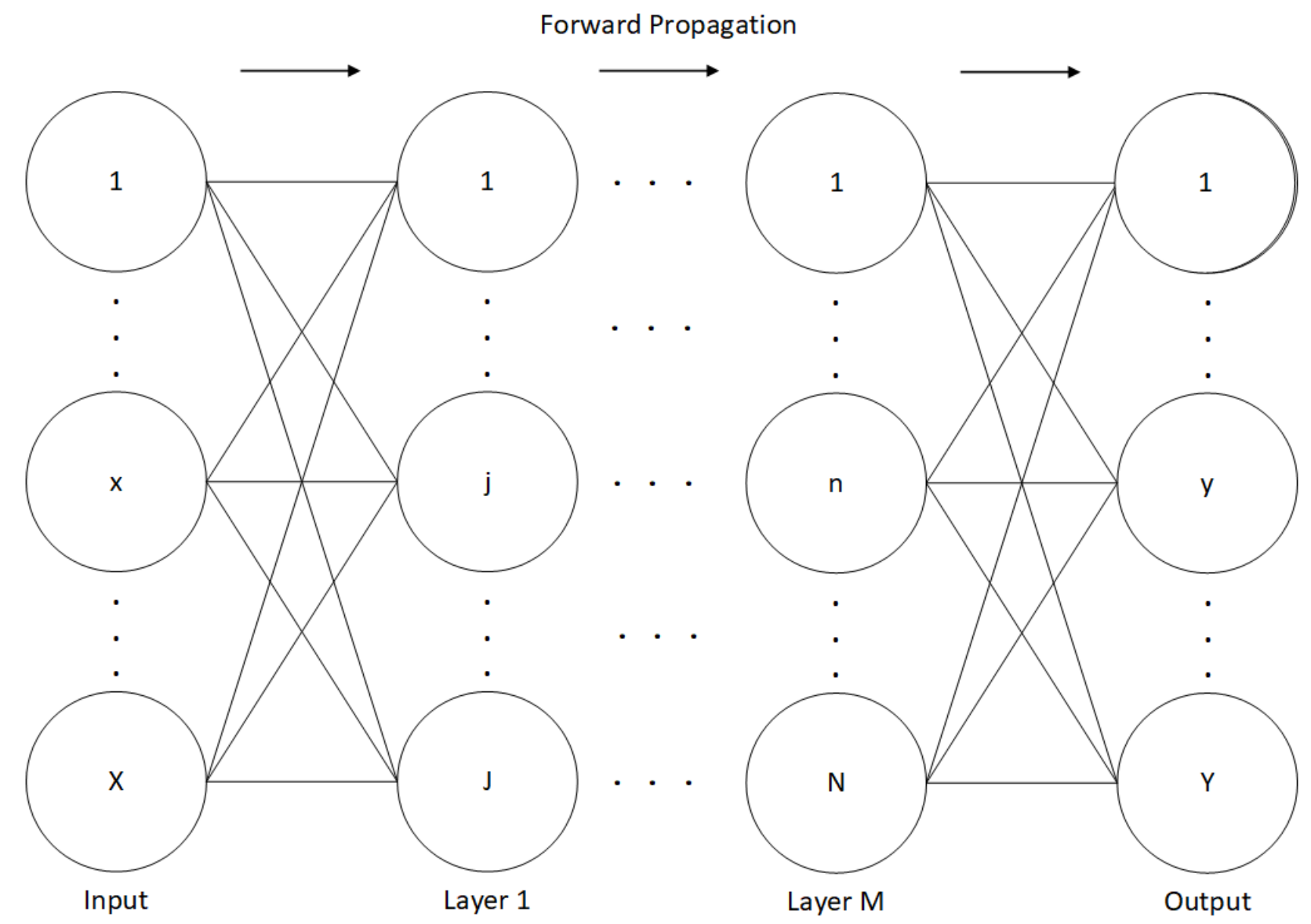

Figure 10: ANN connection structure

this depth was inhibitive due to the computing power required to train Deep Neural Networks (DNNs), but with modern GPUs these networks are becoming much more popular. Specific layer types are discussed below in the context of 2D image recognition.

The image input layer specifies the image size, which is the height, width and channel size (3 for RGB, 1 for grayscale). Pre-processing such as augmentation and normalization also takes place at this layer.

Convolution layers are a set of filters whose responses indicate similarity with the input. These responses enable the CNN to make a decision about the class of the image. 
Along with increases in computing power, much of the recent innovation with DNN's is optimization [16]. One such method is batch normalization. This normalizes the activations and gradients, making training easier and more efficient. It also reduces the sensitivity to initialization parameters. The max pooling operation is used to downsample the images and reduces the feature size. It also removes redundant information. This is crucial as the number of filters at deeper layers can be increased without increased required computational power.

The rectified linear unit (ReLU) is a nonlinear activation layer. It performs a thresholding operation, where any negative value is set to zero. ReLU is another recent addition to $\mathrm{CNN}$, and is easy to compute leading to fast convergence. However, one problem is that neurons can stop activating and basically becomes a useless neuron in the network.

After the convolution and optimization layers, there is one or more of the fully connected layers. This type of layer connects to all the neurons in the previous layer, which combines all the prior features learned. It essentially converts the image input into a vectors for each class. After this process is classifier, which normalizes the output of the last fully connected layer. The softmax classifier consists of an array of positive numbers that sum to one. This is then used by the classification layer to assign the input source to a single class and compute the loss function. For a rigorous mathematical derivation of these layers see a source such as [12] or [17].

\subsection{Literature Review}

A large and growing body of literature has investigated autonomous signal discrimination. [8] provides a comprehensive survey of techniques pre-2007. This work shows two general solutions to modulation recognition: FB and LB, both discussed 
previously in section 2.3. The rest of this review examines the state of current research up to 2018.

Much of the current literature on AMR utilizes one of two FB methods for classification: ANN or decision trees. These studies make use of a time-frequency analysis (TFA) for feature extraction, varying from simple short-time Fourier transform (STFT) to complex nonlinear transformations. [18] uses the statistics of the ChoiWilliams distribution (CWD) combined with a perceptron ANN for classification. In [19], a similar technique is used, which applies a sample averaged input with a CWD transform. This is then classified with a CNN. In [20], a similar but simpler approach is taken, using a STFT and classifying using maximum likelihood.

Another popular approach is the hierarchical decision tree method, as presented in [21], in which the pulses are hierarchical sorted and statistical thresholds are used to classify modulations. First, the instantaneous frequency (IF) is found, from which the standard deviation and kurtosis are calculated. Then the pulses are sorted into two groups from the kurtosis. Finally, they are classified based on the standard deviation. The Wigner Ville distribution is used by [22] to similar effect as [18], with classification being performed by a decision tree. The decision tree used in [22] is based on a normalized centered IF, the standard deviation of the absolute value of the unwrapped phase, and the standard deviation of the median filtered IF profile along with other statistical tests. [23-25] propose similar mechanisms, comparing parameters against empirical thresholds.

Another slightly different HDT classifier is presented in [26]. By using the unwrapped phase, they implement the solution real-time on a Field Programmable Logic Gate (FPGA). This approach, while producing good results, is disadvantageous as the implementation cost would be much higher than a software solution. Additionally, any revisions and updates would be difficult to impossible. 
The studies presented thus far provide evidence that a FB approach can be highly successful in both accuracy and latency. However, the optimum approach in classification is the LB method, in the sense of minimizing false classifications [8]. However, LB classifiers generally have high latency, unless they are simplified and generalized. [27] takes this approach, and presents multiple LB based solutions. It is noted that the probability of classification at lower $(\sim 6 \mathrm{~dB})$ SNR drops significantly for complex signals, which is consistent with other literature [8]. Therefore it is deduced that the FB approach is superior in the modern EM environment, where complex modulations are the norm.

The evidence presented in this section suggests that a real-time software FB approach focusing on low latency and high accuracy is the next step in furthering the field of radar AMC. 


\section{Chapter 3}

\section{Feature and Machine Learning Design}

Three approaches proposed to solve the MOP discrimination problem. There are 13 signals considered for classification:

- Unmodulated

- LFM

- Concave NLFM

- Convex NLFM

- Quadratic NLFM

- Barker Code 13

- Frank Code

- Zadoff-Chu Code

- P1

- P2

- P3

- $\mathrm{P} 4$

- $\operatorname{Px}$

The design architecture for the first approach is presented in fig. 11. It can be considered a feature based HDT/ANN hybrid. The hierarchy flows from the simplest 
pulses to the most complex, with feature extraction and classification happening simultaneously. This ensures that a simple unmodulated pulse will not undergo the same complex signal processing algorithms which are required to classify a PM waveform. At the trees higher levels, the simplest signals are classified with an HDT methodology, using threshold values. Elsewhere further down, a Perceptron type neural network is used to distinguish between PM pulses using TF distributions and Zernike Moments.

Another proposed technique involves just an ANN with minimal feature extraction. This is homogeneous approach, and is generalized for any signal. A different approach which generalizes further is the use of of a CNN for feature extraction and classification. This technique is useful when large labeled data sets are available to train the extraction high quality features. The details on the design and implementation of all three systems follow below.

\subsection{HDT Feature Extraction}

This section lays out the considered features, and the signal processing algorithms required for extraction. They are presented in approximately the same order that they are extracted in, meaning that features describing unmodulated pulses are analyzed first, then separating FM from PM, followed by individual classification of FM. This is approach is intended to minimize computational complexity. 


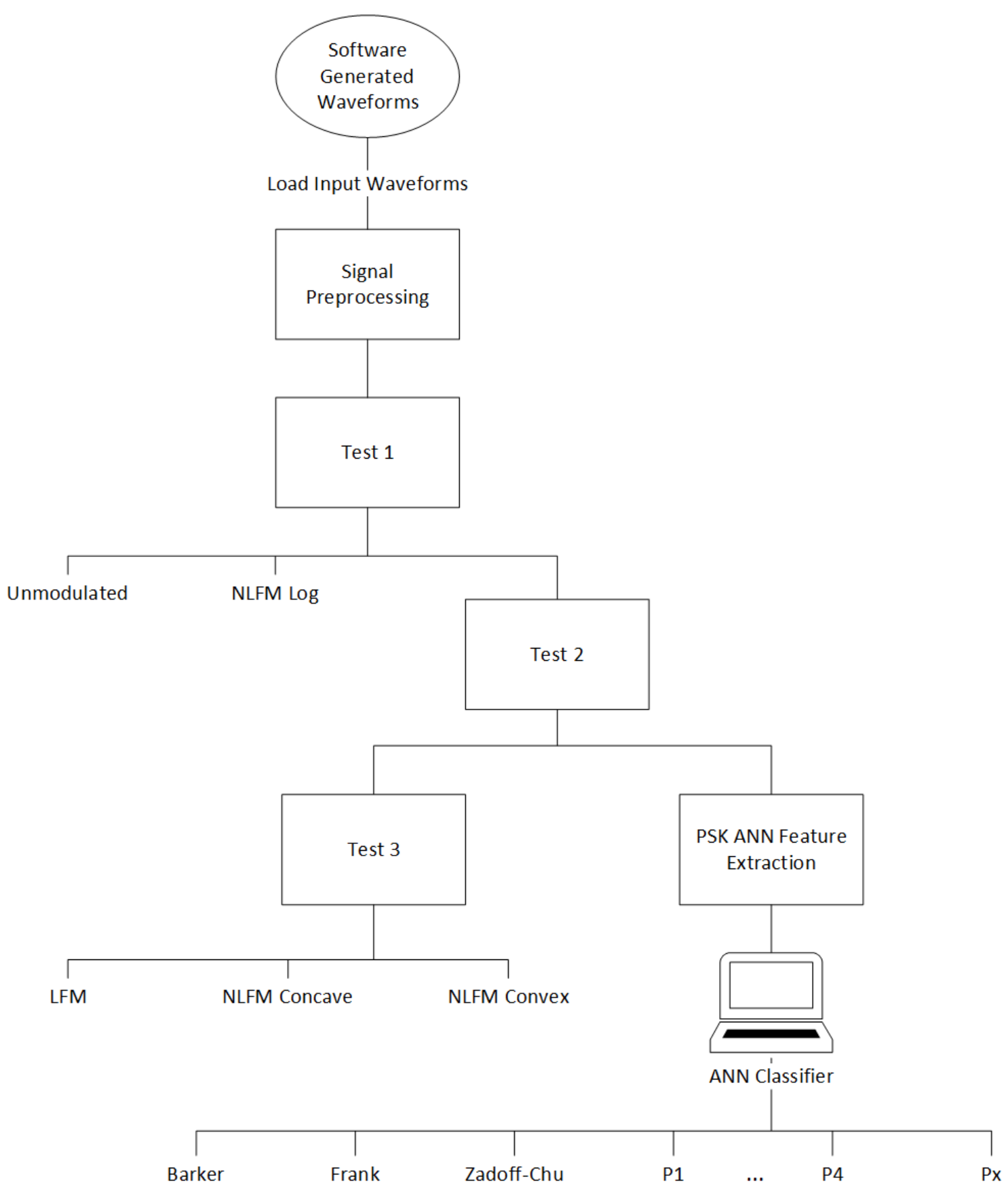

Figure 11: Proposed Architecture for First Design 


\subsubsection{Test 1}

Test 1 is designed to separate the unmodulated and noise from other modulations by recording midpoint crossings $M_{c}$. First each signal is normalized between 0 and 1. This is to ensure that each signal is analyzed with similar amplitudes. An unmodulated pulse is the only type that will cross the midpoint twice, whereas a partial pulse will have one crossing. The NLFM Logarithmic signal is a special case of FM. Examining fig. 27 in appendix A, it is noted that there is one midpoint crossing in the first half of the signal, and many in the second half. This pulse can be classified by breaking the signal in two, and examining the crossings in each half. Any other value of $M_{c}>2$ in the first half indicates another type of pulse modulation. Figure 11 gives the flowchart for the pulse sorting at the first test. 


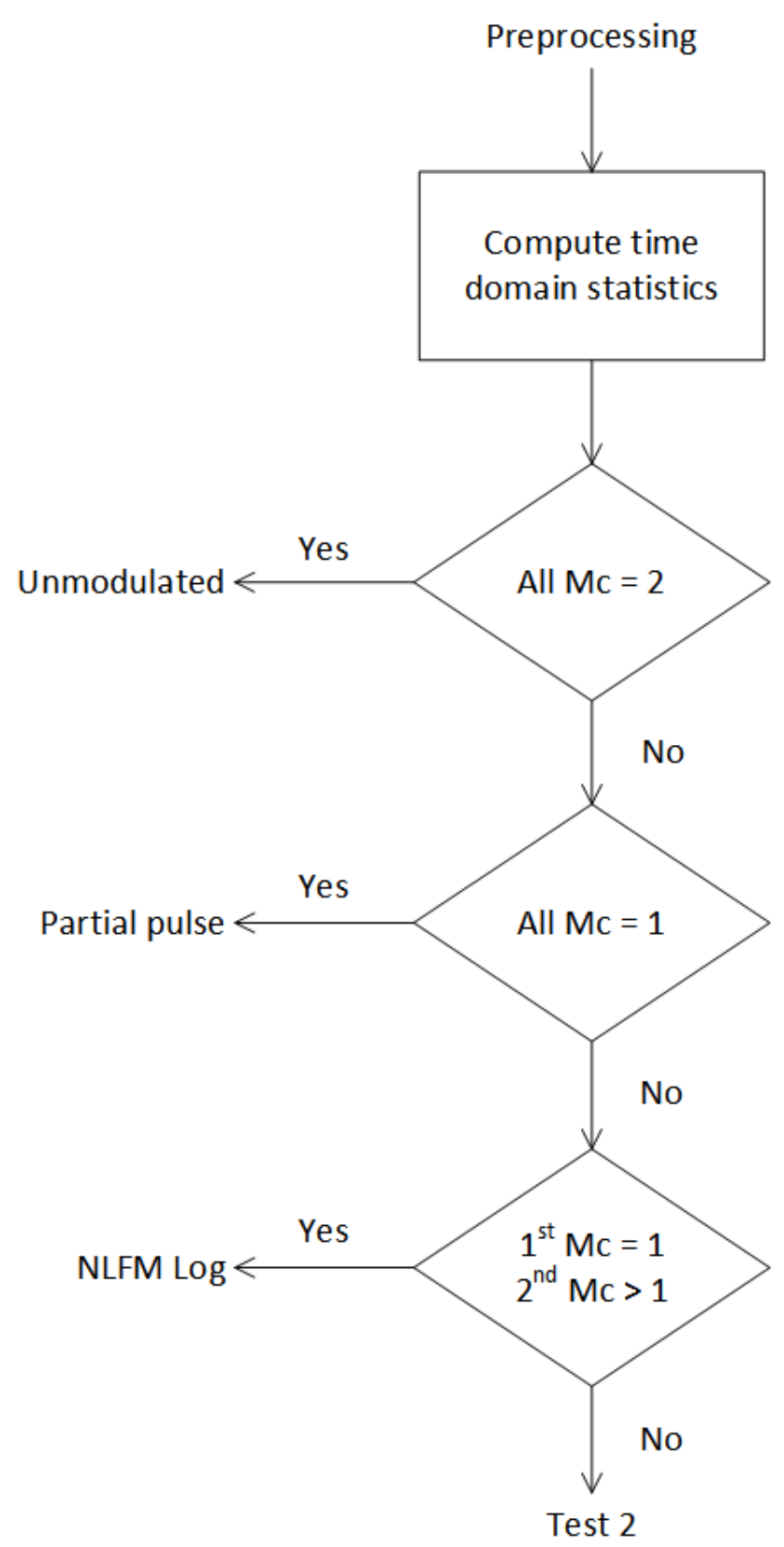

Figure 12: Test 1 Flow Chart 


\subsubsection{Test 2}

In test 2, more complex frequency features are extracted via a Hilbert transform. This transform estimates the instantaneous frequency (IF) of a monocomponent signal. That is, a signal which is described on the time-frequency plan by a single ridge. The rational behind this transform is that the PSK signals will only have the carrier frequency, while the FM signals will show a varying IF. By taking a histogram of the IF, this become very apparent.

The first step is to find the Hilbert transform $h(t)$ of the analytic phasor representation $y(t)$, with respect to the input $x(t)$. Equation (17) expresses the signal model in phasor form, while eq. (18) shows the effect of a Hilbert transform. For the transform's explicit derivation see [28].

$$
\begin{aligned}
& y(t)=A(t) e^{j \phi(t)} \\
& y(t)=x(t)+j h(t)
\end{aligned}
$$

Where $|y(t)|=A(t)$ defines the instantaneous amplitude, and $\arg \{y(t)\}=\phi(t)$ defines the instantaneous phase. [29] Knowing this, the instantaneous frequency $f_{\text {inst }}$ of sample $n$ is computed as the difference between two phase samples and divided by the time step $\delta t=t_{n}-t_{n-1}$, as per eq. (19). Examples of this instantaneous frequency extraction for LFM and Barker 13 are found in fig. 13

$$
f_{i n s t, n}=\frac{\phi\left(t_{n}\right)-\phi\left(t_{n-1}\right)}{\delta t}
$$

The frequency distribution is then examined through a histogram. A histogram is a method of grouping data into bins, wherein the bin count reveals patterns that may not be apparent in the raw data. For analyzing the frequency spectrum, $\kappa$ bins are 


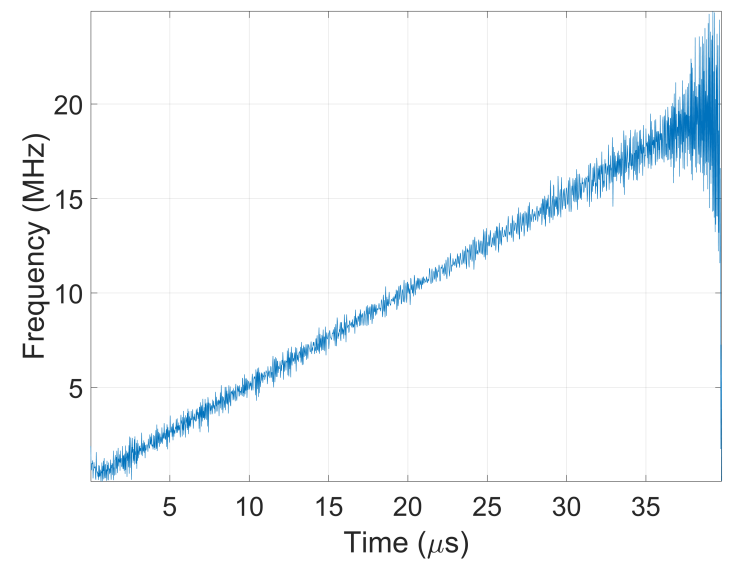

(a) LFM

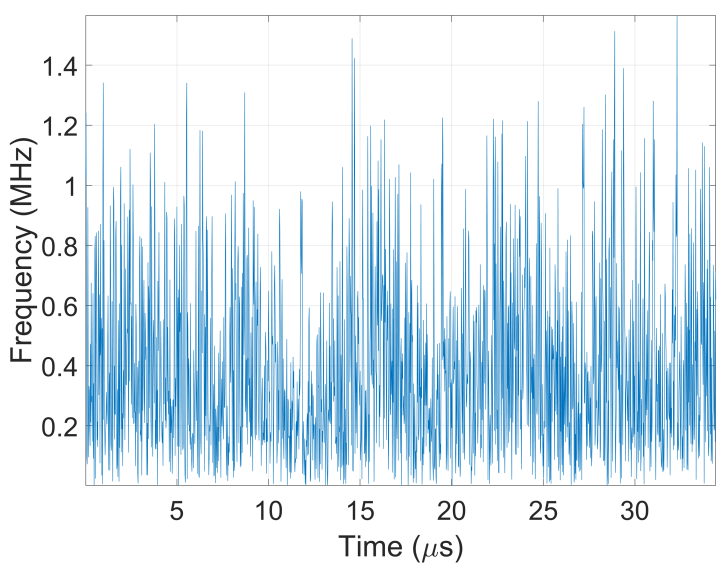

(b) Barker

Figure 13: Instantaneous frequency from Hilbert transform

equally spaced from null to the Nyquist frequency $F_{s}$. As per [29], it is noted that bin width is an important factor affecting the shape of the frequency distribution. If the bins are too wide, features will not be observed, but if they are too narrow, random fluctuations will not be filtered out. A bin count of $\kappa=50$ was chosen empirically, as it emphasized the difference between FM and PSK signals. Figure 14 shows the difference between a LFM and Barker code frequency histogram. PSK codes tend to be very one sided, while LFM is spread over the spectrum. See the simulation setup in chapter 4 for further info on bin width and sampling frequency.

The variance $\sigma^{2}$, and maximum normalized bin count $n_{\max }$ are used for decision thresholds. To find the variance, the mean $\mu$ and generalized moment $m_{i}$ are derived. From here, variance is defined as the second moment. $L$ is the total number of data points in the frequency transform. The relevant equations are presented in eqs. (20) 


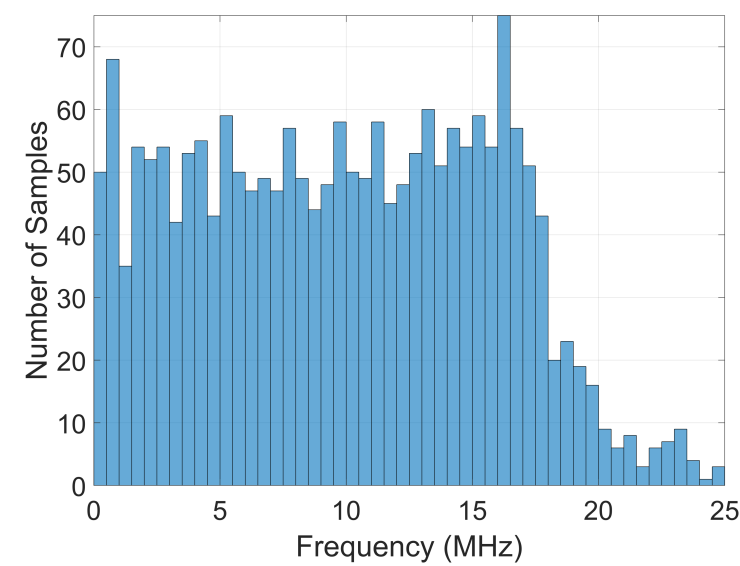

(a) LFM

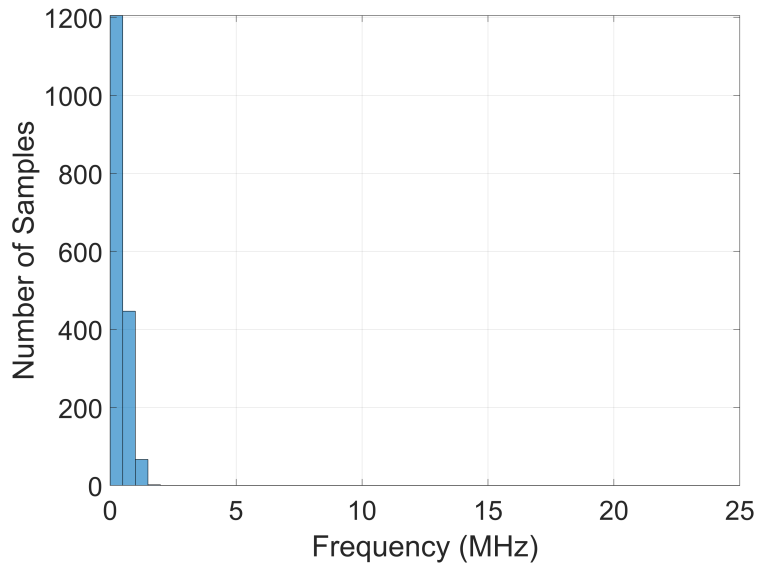

(b) Barker

Figure 14: Frequency histograms with $\kappa=50$

to $(23)$.

$$
\begin{aligned}
\mu & =\frac{\sum_{n=1}^{L} f_{n}}{L} \\
m_{i} & =\frac{\sum_{n=1}^{L}\left(f_{n}-\mu\right)^{i}}{L} \\
\sigma^{2} & =m_{2} \\
n_{\max } & =\frac{\max \left(n_{\kappa}\right)}{L}
\end{aligned}
$$

Figure 15 illustrates the process, with threshold values of 350000 and 0.7 for $\sigma^{2}$ and $n_{\max }$ respectively. 


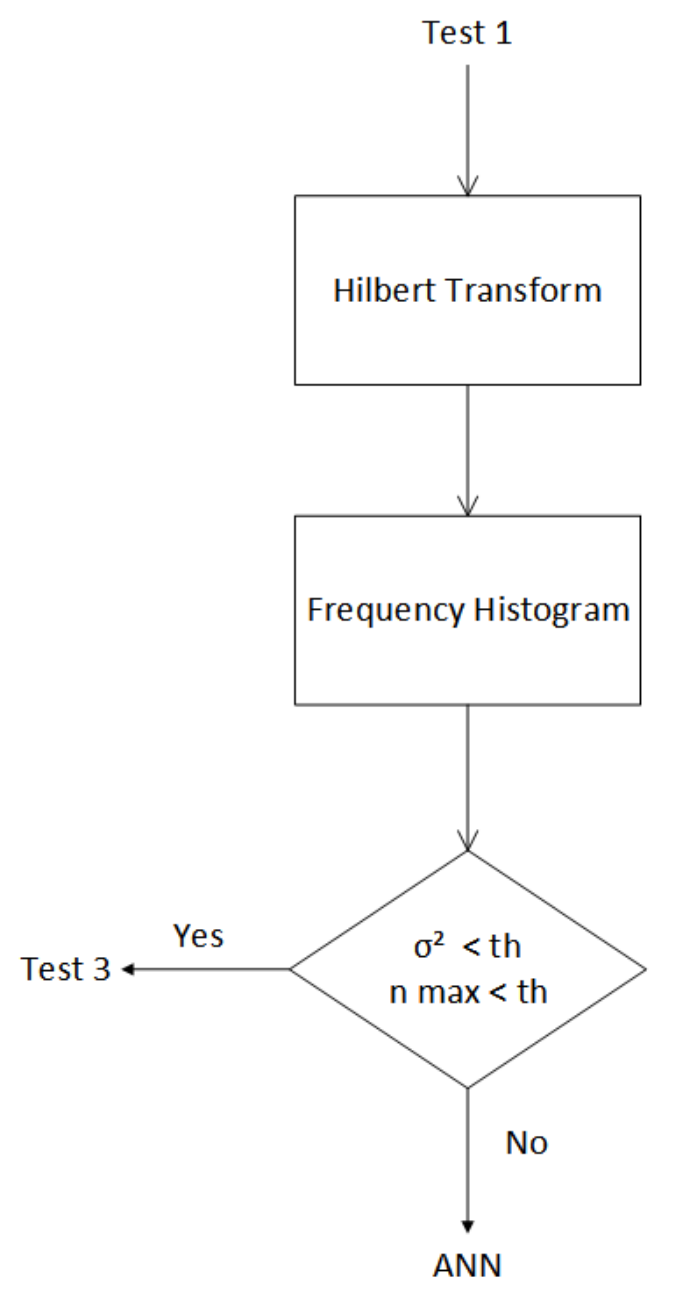

Figure 15: Test 2 Flow Chart 


\subsubsection{Test 3}

The third test uses the IF, as extracted previously, to determine what type of FM is present. Figure 16 presents a general overview of the process. First, two statistics in the frequency domain are extracted: the normalized error $\sigma_{\text {norm }}$ and the fitting error $\sigma_{\text {fit }}$. These are both calculated as in eqs. (24) and (25) using the mean instantaneous frequency, $\mu_{f}$. The fitting error describes how well the frequency follows a linear slope as defined by a beginning frequency $f_{b}$, and end frequency $f_{e}$. Therefore, a LFM pulse will have a fitting error which is less than the normalized error due to noise [24]. This is used as criterion to tag a pulse as LFM.

$$
\begin{gathered}
\sigma_{\text {norm }}=\frac{\sqrt{\sum_{n=1}^{L} f_{n}-\mu_{f}}}{L} \\
\sigma_{f i t}=\frac{\sqrt{\sum_{n=1}^{L}\left(f_{n}-\left(f_{b}+\frac{n-1}{L}\left(f_{e}-f_{b}\right)\right)\right)^{2}}}{L}
\end{gathered}
$$

Next, NLFM type are extracted by examining the slope of the frequency. The pulse is divided into $N$ parts of length $L_{N}$. For each the chirp slope $f_{N}^{\prime}$ is then calculated as per eq. (26). Testing $f^{\prime}$, if $f_{1}^{\prime}<\ldots<f_{N}^{\prime}$ then the NLFM function is concave. Similarly, if the slope is decreasing, as in $f_{1}^{\prime}>\ldots>f_{N}^{\prime}$, the function is convex. Intuitively, this information could also be found examining the second derivative $f_{N}^{\prime \prime}$, which is calculated as per eq. (27).

$$
\begin{aligned}
f_{N}^{\prime} & =\frac{f_{b, N}-f_{e, N}}{L_{N}} \\
f_{N}^{\prime \prime} & =\frac{d^{2} f_{N}}{d t_{N}^{2}}
\end{aligned}
$$

The second derivative is then averaged for each section. This is because the noisy data will effect each value of the $f_{N}^{\prime \prime}$ but will not change the overall trend. If the average 
second derivative is greater than zero for each segment, then the NLFM function is convex. This means that the slope is always increasing, as in a convex function. Similarly, if the average second derivative is less than zero, and therefore the slope is decreasing, the function is concave or logarithmic. If the function fits neither of these profiles, it is declared to be an unknown type of FM. This includes frequency coding modulations and other NLFM waveforms that are not considered. 


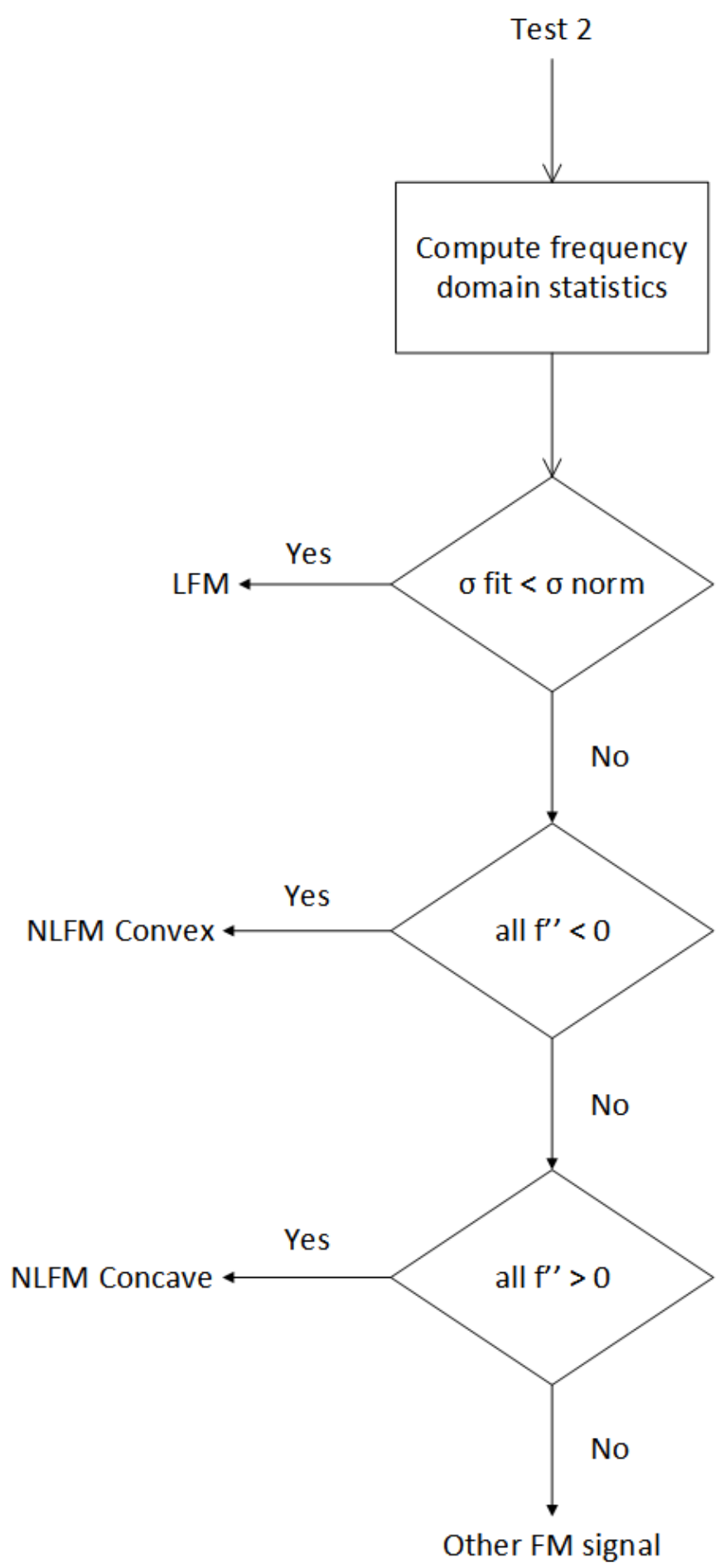

Figure 16: Test 3 Flow Chart 


\subsection{ANN Feature Design}

Care must be taken in designing the inputs to ANN as they suffer from excess dimensionality [11]. It is noted that when there is a fixed number of different training samples, increasing the ANN input dimensionality will first improve the predictive power, then peak and decrease the classifier performance. Therefore, the feature space chosen must only include information which is useful in separating the PSK signals.

\subsubsection{Time Frequency Transforms}

A number of Time-Frequency (TF) transforms were studied for their usefulness in distinguishing between various types of MOPS.

The Pseudo Wigner Ville Distribution (PWVD) is defined in eq. (28) as per [30]. The full derivation is found in [30].

$$
h_{p w v d}(t, f)=\int h(\tau) e^{-j 2 f \tau} y\left(t+\frac{\tau}{2}\right) y^{*}\left(t+\frac{\tau}{2}\right) d \tau
$$

The CWD has an exponential kernel, which can be easily adjusted by the scaling parameter $\sigma$. The transform is defined in eq. (30), with the full derivation found in [31].

$$
h_{c w}(t, f)=\iint \frac{\sqrt{\sigma}}{4 \sqrt{\pi}|\tau|} e^{-f^{2} \rho /\left(16 \tau^{2}\right)} y\left(t+f+\frac{\tau}{2}\right) y^{*}\left(t+f+\frac{\tau}{2}\right) d f d \tau
$$

First the frequency is normalized by subtracting the minimum, and then dividing by the maximum. This produces value ranging from zero to one. Next, time domain and other features are extracted. 


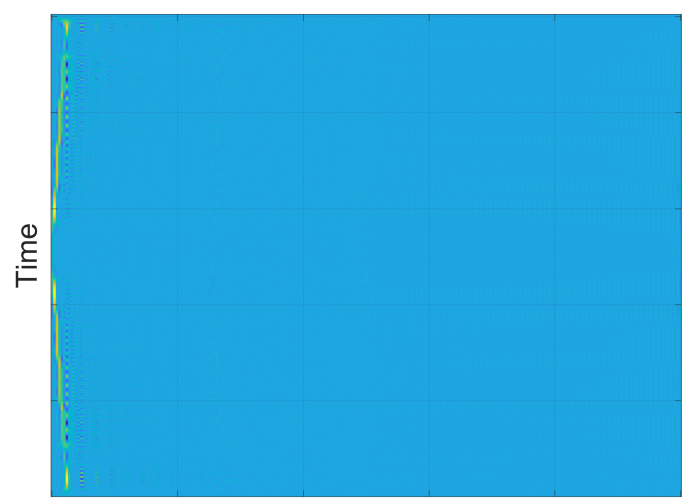

Frequency

(a) P1 after transform

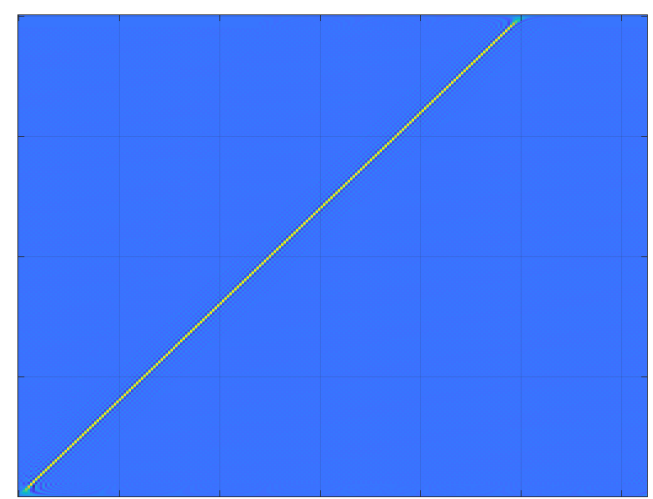

(b) LFM after transform

Figure 17: $\mathrm{P} 1$ and LFM spectrograms using CWD with $\sigma=10$

From this transform, a two dimensional image is obtained in which the changes in frequency can be analyzed with respect to time. An comparison of the modulation images obtained using the raw transform is presented in Figure 17. The PSK signals examined each have a unique signature, needs to be described.

\subsubsection{Image Analysis and Features}

In the following analysis, the $\mathrm{TF}$ data is treated as an image. Before extracting useful information about the shape, the image must be preproccessed through the following steps:

1. Global thresholding: removes areas that are not part of the main feature

2. Time and frequency gating: removes noise and artifacts from the transform on the edges of the image

3. Image resizing: normalizes the aspect ratio to a 1:1 square image

Figure 18 shows the difference between the raw CWD and the fully processed image. This processing is necessary as any noise or artifacts in the final image can lead to 


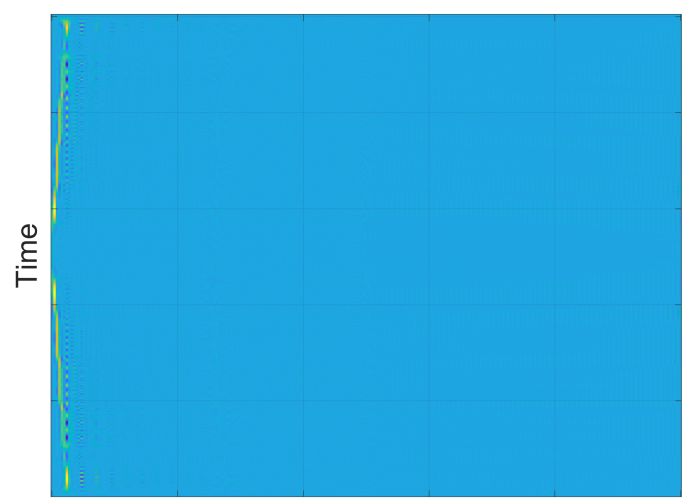

Frequency

(a) Before processing

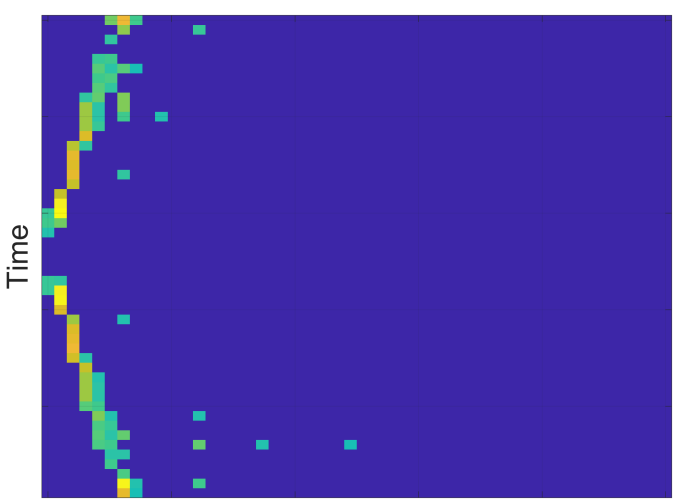

Frequency

(b) After processing

Figure 18: $\mathrm{P} 1$ spectrogram using CWD with $\sigma=10$

wrong shape information, resulting in misclassifications. Basic global thresholding is accomplished as per the algorithm in [32]. This produces a binary image, which is gated in step 2. Gating entails removing all columns or rows without any information, that is a value of 0 . At this point all rows and columns of the image contain at least 1 non-zero pixel. In step 3, the image is resized to an aspect ratio of 1:1 using nearest-neighbor interpolation. This process entails assigning the nearest original pixel value to the new output pixel. Nearest-neighbor interpolation is chosen due to its speed, as the quality of a binary image is minimally affected [32]. Now that the TF representation is standardized, the shape can be considered.

It has been proven that most of an images information can be captured by a big enough set of moments. There are many types of moments, and those that are invariant to image transformations, such as translation, scaling and rotation, are invaluable for pattern classification. These features will give consistent values that are less susceptible to MOP shape variation from parameters such as SNR, PW and BW. The complex pseudo-Zernike moment (PZM) is defined in eq. (31), and was selected 
for the good performance at low SNR compared to other techniques. The normalized error for total image reconstruction using PZMs is at or near minimum between moments of order 5 to 25 [33]. The amount used was varied and experimentally determined, and is discussed in Chapter 4.

$$
\begin{aligned}
Z_{n, m} & =\frac{n+1}{\pi} \sum_{n-s-m=e v e n}^{n-|m|} D_{n m s} \sum_{a=0}^{k} \sum_{b=0}^{m}(-j)^{b}\left(\begin{array}{c}
k \\
a
\end{array}\right)\left(\begin{array}{c}
m \\
b
\end{array}\right) B_{n m s} G_{2 k-2 a+m-b, 2 a+b} \\
& +\frac{n+1}{\pi} \sum_{n-s-m=o d d}^{n-|m|} D_{n m s} \sum_{a=0}^{d} \sum_{b=0}^{m}(-j)^{b}\left(\begin{array}{c}
d \\
a
\end{array}\right)\left(\begin{array}{c}
m \\
b
\end{array}\right) B_{n m s} R_{2 d-2 a+m-b, 2 a+b}
\end{aligned}
$$

where $k=\frac{n-s-m}{2}$ and $d=\frac{n-s-m-1}{2}$. The radial polynomial terms $R_{n m}, D_{n m s}$ and $B_{n m s}$ and the scale invariant central moment $G_{p q}$ are derived in [33]. Note that $Z_{n, m}$ is a complex value which contains rotation information. The moment is made invariant by taking the absolute value, that is $Z_{\text {invn,m }}=\left|Z_{n, m}\right|$. 


\section{Chapter 4}

\section{Simulation Setup}

The simulation was coded in MATLAB on versions R2017b and R2018a. Along with standard MATLAB toolboxes, the Time-Frequency Toolbox [34] created by CNRS and Rice University, and the Fast and Memory-Efficient Algorithms for Quadratic Time-Frequency Distributions [35] were utilized. A radar signal test data generator (RSG), also coded in MATLAB, was developed by D-TA Systems Inc. to provide the data set on which the AMC design could be tested and validated.

\subsection{Signal Generation}

Each type of modulation was tested, trained, and validated with varying $\mathrm{PW}, \mathrm{BW}$, and SNR. The software based signal generator ensures that each signal has different Additive White Gaussian Noise (AWGN) and therefore can be considered unique. Equation (32) shows the basic signal model that is considered for any discrete data point $n$.

$$
y_{n}=x_{n}+w_{n}+z_{n}
$$


Table 1: Constant Signal Generator Parameters

\begin{tabular}{ll}
\hline Sampling Frequency & $50 \mathrm{MHz}$ \\
Transmit Antenna & 16 Element Isotropic Linear Array \\
Receive Antenna & 17 Element Isotropic Circular Array \\
\hline
\end{tabular}

where $y_{n}$ is the received waveform, $x_{n}$ is the transmitted waveform, $w_{n}$ is AWGN, and $z_{n}$ is environmental considerations such as Rayleigh fading and Doppler effects. The transmitted pulse is selected to be one of the thirteen signals discussed in Chapter 3. While the signal generation is designed to mimic a real scenario as much as possible, some parameters were kept constant. These are listed in table 1.

\subsection{Input and Preprocessing}

The waveforms are input as a transmit and receive pulse chain with both inphase data, I, and quadrature data, Q. The characteristics of each data file, such as number of pulses and MOP, have been pre-determined in the signal generation software. Each file is loaded as a signal class object, as defined in Appendix B. Through the functions associated with the signal class, the IQ is separated, and additional AWGN or Doppler effects are optionally added. Next, the pulse chains are separated into individual pulses through a Hilbert transform and moving exponential average. This gives a marker for the start and end of each pulse. At this point the signal is ready for feature extraction and classification. 


\subsection{Feature Extraction}

The feature extraction is performed through two functions: HDTfeatExt and ANNfeatExt. These in turn are part of the signal class. See Algorithms 4 and 5 in Appendix B for the full code. There is a variation on this code $A N N$ featExtPar, which uses different syntax to enable parallel processing of the features. Instead of taking one pulse and performing the processing, these functions take a block of code with a list of pulse markers. Each pulse is then identified via the markers, and sent to a different processor in the computing pool. Once an individual processor finishes with the current pulse, the next pulse in queue is taken for analysis. The advantage is reduced processing times. Algorithm 1 shows the differences in code for ANNfeatExtPar, which occur at the beginning of the function.HDTfeatExt is less computationally intensive, so parallel computing was not implemented. See Chapter 5 for a full comparison on processing times.

\section{Algorithm 1: ANNfeatExtPar differences}

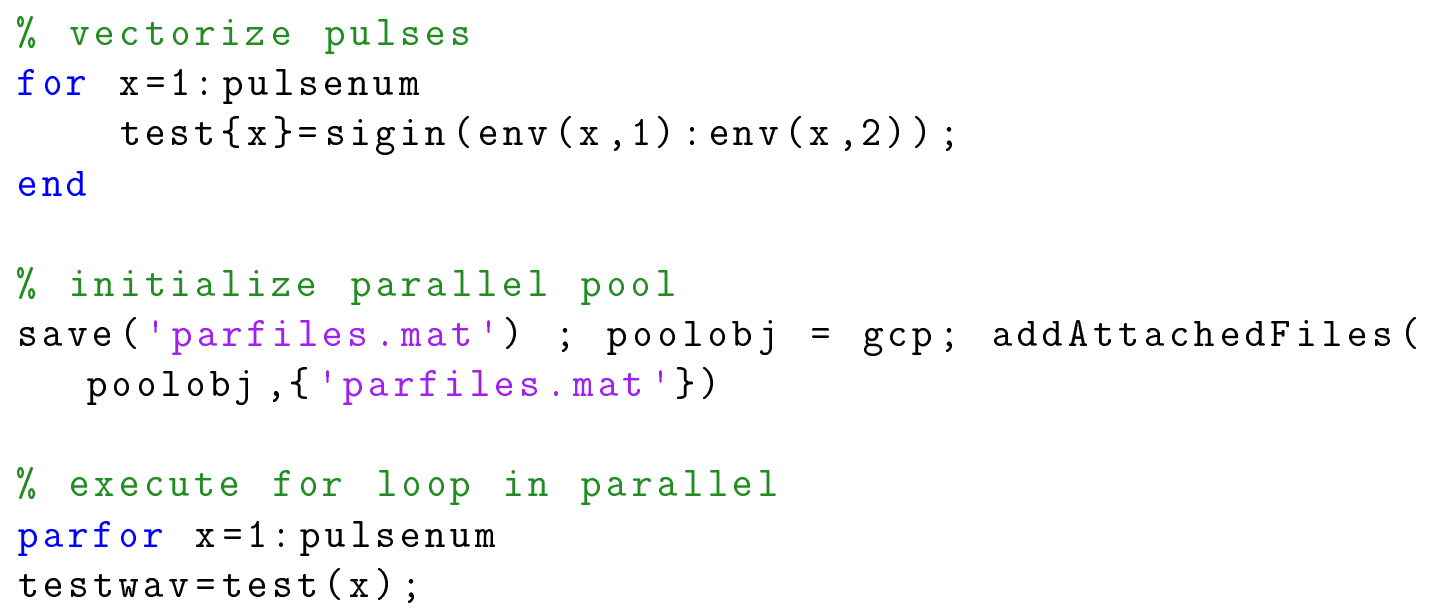

While many features were extracted from the pulses, only some proved useful in classification. Features were selected on the basis of maximizing mutual information between classes. The list of features considered for the HDT/ANN hybrid is as follows:

1. Midpoint crossings $M_{c}$ 
2. Instantaneous frequency from Hilbert Transform $f_{\text {inst }, n}$

3. Frequency normalized error $\sigma_{\text {norm }}$

4. Frequency fitting error $\sigma_{f i t}$

5. First derivative of frequency $f^{\prime}$

6. Second derivative of frequency $f^{\prime \prime}$

7. Time domain WT

8. TF WT

9. TF CWD

10. TF PWVD

11. Zernike moments $Z_{m, n}$

The following features were considered for the ANN classifier:

1. TF WT

2. TF CWD

3. TF PWVD

4. Zernike moments $Z_{m, n}$

The CNN classifier only considers the TF PWVD transform, mapped and formatted to a $224 \times 224 \times 3$ color image. 


\subsection{Neural Network Training and Parameters}

\subsubsection{ANN Setup}

Back propagation based offline training was implemented for the ANN, using functions from MATLABs machine learning toolbox. Creating these models offline utilizes available computing power to minimize classification error. One ANN is trained for each type of MOP; this creates a library of models to compare new signals against. The network parameters are set as:

- Input nodes: a constant number which is equal to the length of the feature vector to be analyzed

- Hidden layer nodes: varied from a minimum of 5 , to a maximum of 30

- Output nodes: constant number, set to the number of classification categories

- Training algorithm: Scaled conjugate gradient backpropagation, see [36] for details

Each set of training data is split into 3 sections with the following ratios: $70 \%$ training, $15 \%$ validation, and 15\% testing. The validation set is used in early stopping, which prevents overfitting. The training stops when any of the following conditions are reached:

- Maximum number of epochs reaches 1000

- Validation performance worsens 6 times in a row (early stopping)

- Performance is minimized to a cross entropy of zero

- The performance gradient falls below 1e-6

The performance is calculated through calculating the individual cross entropy $C E_{m}$ between the output $y_{m}$, which is the ANN estimated class, and the target $t$, which is the real class. Note that $y$ is a represents the percentage estimate that a pulse belongs to a class $m$, and sums to one across all classes $M$. Perfect classification performance 
minimizes the total $P_{C E}$ across all classes to zero, while poor performance results in larger results. Cross entropy operates on a log scale, as per eq. (33), which means the classifier penalizes inaccurate classifiers much more than ones that have minor inaccuracy. More details on cross entropy are available in [36].

$$
\begin{gathered}
C E_{m}=-t \log (y) \\
P_{C E}=\frac{\sum_{m=1}^{M} C E_{m}}{N}
\end{gathered}
$$

where $N=n_{\text {pulses }} M$, which is the total number of elements in the cross entropy array. This setup in encapsulated in the function $A N N_{-}$Training. Having multiple different models for each MOP is useful as they can be used in committee, with the majority being used for classification. Chapter 3 discusses majority decision voting in detail. The purpose of this function is to automatically train models, and to only keep the ones of the highest quality. This is accomplished through three factors, analyzed after a model is trained:

- Maximum cross entropy performance of $P_{C E, \max }=0.02$

- Adaptive incremental performance threshold $P_{C E, a d t}=P_{C E, b e s t}$

- Number of models to keep set to 15

- Maximum number of failed models is 200

If a model does not meet the maximum or adaptive threshold, it is discarded. The maximum is simple: the final model performance must be lower than the set threshold of 0.02 . The adaptive threshold takes the best models performance and sets it to be the $P_{C E, a d t}$. If a new better model cannot be found within 50 iterations, $P_{C E, a d t}$ is increased by $20 \%$ and model training continues. If there is 200 failures in a row, then the training is stopped. This therefore ensures the best 15 models are produced for each MOP. The header for this code is presented Algorithm 2, with the full function 
in Appendix B, Algorithm 6.

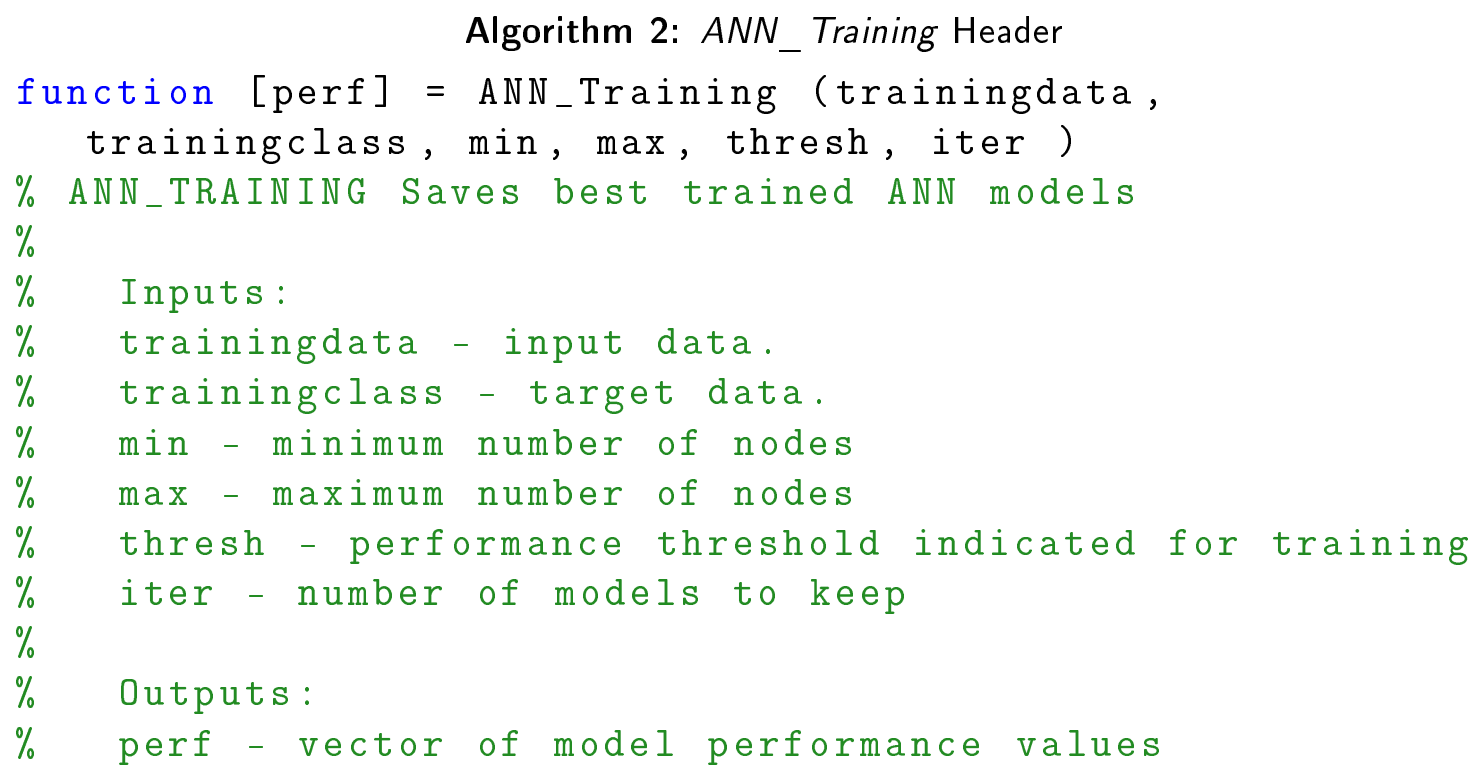

\subsubsection{CNN Setup}

Training for the CNN utlizes stochastic gradient descent with momentum (SGDM). This method is chosen due to its fast convergence rate. Unlike the ANN discussed previously, where multiple models are required to be trained, one model with many layers are trained in this method. Each set of training data is split into 3 sections with the following ratios: $90 \%$ training, 10\% validation. Testing is only performed after a model is trained. Using the high end NVIDIA Tesla P100 GPU the training parameters are defined as:

- Maximum number of epochs is 3

- 45 iterations per epoch

- Validation every 30 iterations

- Validation performance worsens 6 times in a row (early stopping)

- Performance is minimized to a cross entropy of zero 
Table 2: Layer Setup

\begin{tabular}{ccc}
\hline Number & Layer Type & Properties \\
1 & Input & $224 \times 224 \times 3$ input with zerocenter normalization \\
2 & Convolution & $163 \times 3 \times 3$ convolutions \\
3 & Batch Normalization & Batch normalizer with 16 channels \\
4 & ReLU & Standard ReLU \\
5 & Max Pooling & $2 \times 2$ max pooling layer \\
6 & Convolution & $323 \times 3 \times 16$ convolutions \\
7 & Batch Normalization & Batch normalizer with 32 channels \\
8 & ReLU & Standard ReLU \\
9 & Max Pooling & $2 \times 2$ max pooling layer \\
10 & Convolution & $643 \times 3 \times 32$ convolutions \\
11 & Batch Normalization & Batch normalizer with 64 channels \\
12 & ReLU & Standard ReLU \\
13 & Fully Connected & Fully connected layer with 13 nodes \\
14 & Softmax & Softmax classifier with 13 outputs \\
15 & Output & Classification output \\
\hline
\end{tabular}

An iteration is defined as one step towards minimizing the loss using a part of the data, whereas a epoch is a full pass of the algoritym over the whole training set. The validation and performance is the same as detailed in section 4.4.

The model is setup with fifteen layers, with the input accepting a 224x224x3 RGB image, and outputting 1 of 13 classes. Details on each layer are presented in table 2 . 


\section{Chapter 5}

\section{Results}

In this chapter, the design, as presented in Chapter 3, was set up and simulated as per Chapter 4. The performance of the proposed solutions are first examined under varying noise and subpulse conditions, and then compared to each other. Additionally, the effects of varying carrier frequency $\left(f_{c}\right)$ is analyzed. Sources of error and possible improvements are also discussed. The test cases considered are listed in table 3, and will be analyzed to determine system performance and shortcomings. Note that each dataset contained consisted of 9900 pulses, evenly distributed among each modulation type.

Table 3: Subpulse Parameters

\begin{tabular}{ccccc}
\hline Dataset & SNR $(\mathrm{dB})$ & $\mathrm{BW}(\mathrm{MHz})$ & $\mathbf{P W}(\boldsymbol{\mu s})$ & $\boldsymbol{f}_{\boldsymbol{c}}(\mathbf{G H z})$ \\
Training & $12-30$ & $10-20$ & $25-100$ & 9 \\
Test 1 & $-6-30$ & 20 & 100 & 9 \\
Test 2 & $-6-30$ & 10 & 25 & 9 \\
Test 3 & $-6-30$ & $1-40$ & $5-100$ & 9 \\
Test 4 & $-6-30$ & $1-40$ & $5-100$ & 0.5 \\
Test 5 & $-6-30$ & $1-40$ & $5-100$ & 5 \\
\hline
\end{tabular}




\section{$5.1 \quad \mathrm{HDT} / \mathrm{ANN}$ Classification}

The performance of the classifier is detailed with a test case 1 in table 4 . The pulses at vary from $30 \mathrm{~dB}$, which is essentially no noise, to $-6 \mathrm{~dB}$, considered very noisy by ELINT standards. The PW and BW values are kept static. The HDT achieves $100 \%$ classification accuracy in an ideal environment. This proves that the design works in theory, but the noise presents significant problems. Even with various filters and methods to make the pulses noise tolerant, the performance quickly drops. The classifier was non-effective in a generalized environment when the subpulse parameters were changed, such as in test 2 and 3. Performance was essentially zero at all SNRs.

The ANN was trained on both the CW and PWVD transforms, using the subset of PSK waveforms from the training dataset. The results are similar, with the CW selected as it performed slightly better. From the CW transform the first 42 Zernike moments, the standard deviation of frequency, the first two moments in the time domain, and the time with maximum power in the TF spectrum are extracted. This is what was used in the final system. However much of the incorrect classification occurred in the first part of the system, almost rendering the ANN section useless. The ANN performance was noted to have a higher accuracy at all SNRs when used outside of the HDT architecture, which is what the next method accomplishes. 
Table 4: HDT/ANN Classifier Performance for Test 1

\begin{tabular}{ccccccc}
\hline MOP & $\mathbf{- 6} \mathrm{dB}$ & $\mathbf{0} \mathrm{dB}$ & $\mathbf{6} \mathrm{dB}$ & $\mathbf{1 2} \mathbf{d B}$ & $\mathbf{2 4} \mathbf{d B}$ & $\mathbf{3 0} \mathbf{d B}$ \\
None & 0 & 0.95 & 1 & 1 & 1 & 1 \\
LFM & 0 & 0 & 0 & 0 & 0.22 & 1 \\
Concave & 0 & 0 & 0 & 0 & 0.74 & 1 \\
Convex & 0 & 0 & 0 & 0 & 0 & 1 \\
Log & 0 & 0 & 0 & 0 & 0.94 & 1 \\
Barker & 0 & 0 & 0 & 0 & 0 & 1 \\
Zad-Ch & 0.16 & 0.13 & 0 & 0 & 0 & 1 \\
Frank & 0.89 & 0.97 & 0.24 & 0 & 0 & 1 \\
P1 & 0 & 0 & 0 & 0 & 0 & 1 \\
P2 & 0.42 & 0 & 0 & 0 & 0 & 1 \\
P3 & 0 & 1 & 1 & 1 & 1 & 1 \\
P4 & 0 & 0 & 0 & 0 & 0 & 1 \\
Px & 0 & 0 & 0 & 0 & 0 & 1 \\
Overall & 0.11 & 0.23 & 0.17 & 0.15 & 0.3 & 1 \\
\hline
\end{tabular}




\subsection{ANN Classification}

The ANN was trained using PWVD transforms to generate a vector of 46 features per pulse. PWVD was used in this case due to its computational efficiency, as the training dataset was much larger when considering all MOPs. The features are the same as in the HDT/ANN hybrid; that is the first 42 Zernike moments, the standard deviation of frequency, the first two moments in the time domain, and the time with maximum power in the TF spectrum.

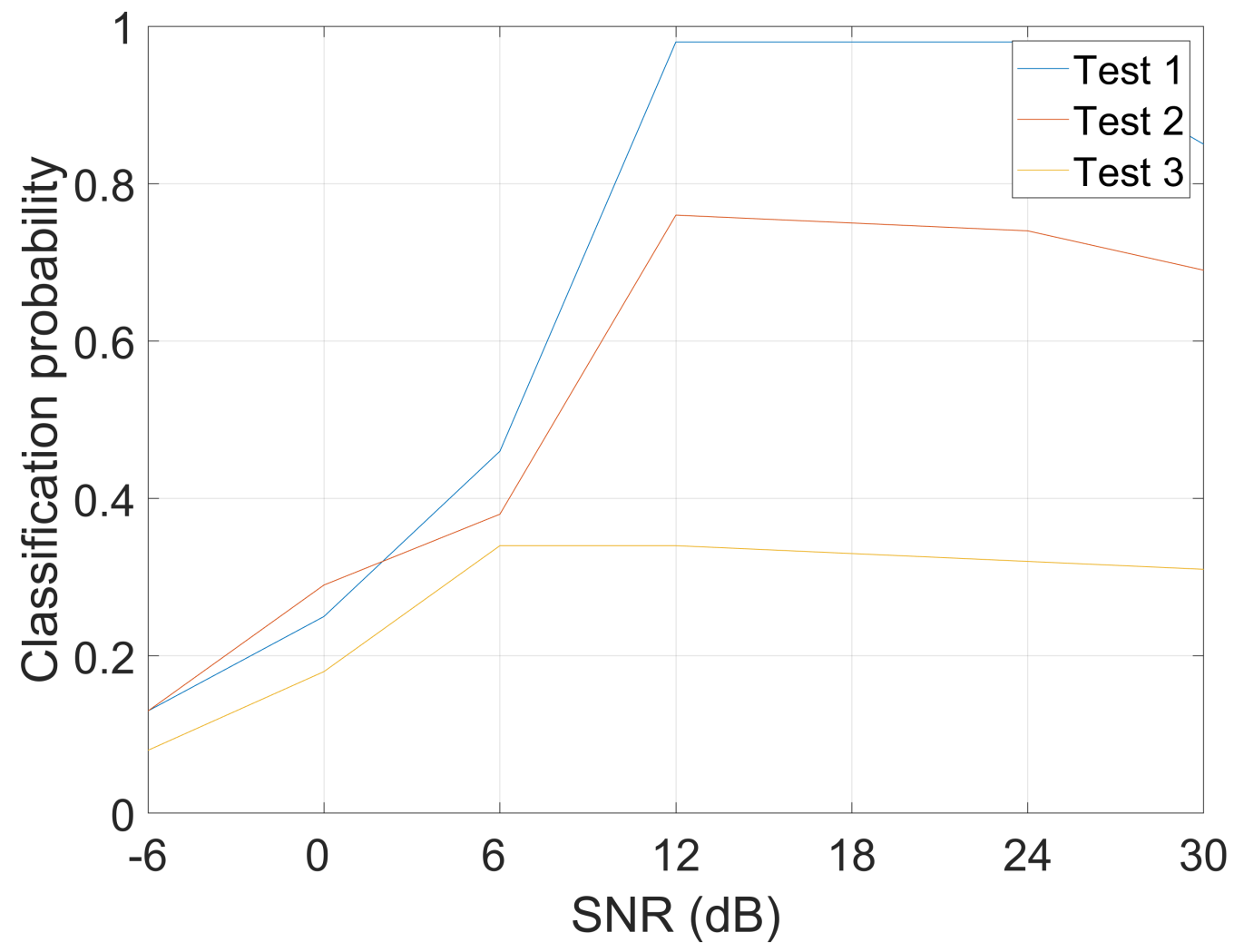

Figure 19: Overall ANN Classification Performance

Figure 19 presents the overall classification performance for test 1 , test 2, and test 3. Test 1 performs perfect at high SNR values, suffering at SNRs less than the training dataset. When training was attempted at lower SNRs the classifier 
had lower overall performance at any SNR. This is likely due to very degraded signals having similar characteristics and being hard to distinguish. Test 2 has a classification performance of close to $80 \%$ at high SNR, with similar performance to test 1 at low SNR. This is likely due to problems with the shorter PW of test 2. As per table 6 this was mainly a problem with the NLFM pulse types, with some confusion in the P codes. Examining appendix C.2, the confusion matrices show that these pulses are incorrectly classified as LFM or unmodulated. This is noted throughout the tests, as similar pulse types end up being clustered together. This clustering is still useful, as the main separation between PSK and FM codes is very apparent. For example, examining the confusion matrix for test 1 at an SNR of $0 \mathrm{~dB}$ (fig. 43), the FM waveforms cluster around unmodulated, LFM, and concave. However, the PSK are mainly split between Zadoff-Chu, Frank, P2 and P4 codes.

Test 3 proposes a more realistic scenario with varied subpulse parameters that are both inside and outside the training range. This is designed to see how well the system will generalize to an unpredictable environment; results suggest poor performance. While low SNR gives similar, yet lower performance, high SNR has a correct classification rate that is just over $30 \%$. This entails that a different method of classification or feature extraction is required to classify pulses in a realistic EM situation. The same clustering of pulses as discussed for test 2 is apparent for test 3 . The confusion matrices can be examined in appendix C.3.

Test 4 and 5 examine the performance with respect to center frequency variation. The subpulse parameters are kept identical to test 3. As per fig. 20, the performance is essentially invariant with changes in carrier frequency. While the classifier confusion on individual pulses changed, the overall accuracy was very similar. This 
Table 5: ANN Classifier Performance for Test 1

\begin{tabular}{ccccccc}
\hline MOP & $-\mathbf{6} \mathrm{dB}$ & $\mathbf{0} \mathrm{dB}$ & $\mathbf{6} \mathrm{dB}$ & $\mathbf{1 2} \mathrm{dB}$ & $\mathbf{2 4} \mathbf{d B}$ & $\mathbf{3 0} \mathrm{dB}$ \\
None & 1 & 1 & 1 & 1 & 1 & 1 \\
LFM & 0 & 0.06 & 1 & 1 & 1 & 1 \\
Concave & 0 & 0.32 & 0.05 & 0.93 & 0.9 & 1 \\
Convex & 0 & 0 & 0 & 0.99 & 1 & 0 \\
Log & 0 & 0.05 & 0.98 & 1 & 1 & 1 \\
Barker & 0 & 0 & 0.28 & 1 & 1 & 1 \\
Zad-Ch & 0.01 & 0.97 & 0.99 & 1 & 1 & 1 \\
Frank & 0 & 0 & 0.53 & 1 & 1 & 1 \\
P1 & 0 & 0 & 0.49 & 1 & 1 & 1 \\
P2 & 0 & 0 & 0.02 & 1 & 0.98 & 1 \\
P3 & 0 & 0 & 0.01 & 0.99 & 1 & 1 \\
P4 & 0.71 & 0.94 & 0.67 & 1 & 0.98 & 1 \\
Px & 0 & 0 & 0 & 0.87 & 0.84 & 0 \\
Overall & 0.13 & 0.25 & 0.46 & 0.98 & 0.98 & 0.85 \\
\hline
\end{tabular}


Table 6: ANN Classifier Performance for Test 2

\begin{tabular}{ccccccc}
\hline MOP & $-\mathbf{6} \mathrm{dB}$ & $\mathbf{0} \mathrm{dB}$ & $\mathbf{6} \mathrm{dB}$ & $\mathbf{1 2} \mathrm{dB}$ & $\mathbf{2 4} \mathbf{d B}$ & $\mathbf{3 0} \mathrm{dB}$ \\
None & 1 & 1 & 1 & 1 & 1 & 1 \\
LFM & 0.02 & 0.9 & 1 & 1 & 1 & 1 \\
Concave & 0.01 & 0.01 & 0 & 0 & 0 & 0 \\
Convex & 0 & 0 & 0 & 0 & 0 & 0 \\
Log & 0 & 0 & 0 & 0 & 0 & 0 \\
Barker & 0 & 0 & 0.29 & 1 & 1 & 1 \\
Zad-Ch & 0 & 0.98 & 0.98 & 1 & 1 & 1 \\
Frank & 0 & 0 & 0.56 & 1 & 1 & 1 \\
P1 & 0 & 0 & 0.47 & 0.99 & 0.66 & 1 \\
P2 & 0 & 0 & 0.01 & 0.99 & 1 & 1 \\
P3 & 0 & 0 & 0.01 & 1 & 0.99 & 1 \\
P4 & 0.68 & 0.92 & 0.67 & 0.99 & 1 & 1 \\
Px & 0 & 0 & 0 & 0.83 & 1 & 0 \\
Overall & 0.13 & 0.29 & 0.38 & 0.76 & 0.74 & 0.69 \\
\hline
\end{tabular}


Table 7: ANN Classifier Performance for Test 3

\begin{tabular}{ccccccc}
\hline MOP & $\mathbf{- 6} \mathrm{dB}$ & $\mathbf{0} \mathrm{dB}$ & $\mathbf{6} \mathrm{dB}$ & $\mathbf{1 2} \mathbf{d B}$ & $\mathbf{2 4} \mathbf{d B}$ & $\mathbf{3 0} \mathrm{dB}$ \\
None & 0.62 & 0.97 & 1 & 1 & 1 & 1 \\
LFM & 0 & 0.02 & 0.92 & 1 & 1 & 1 \\
Concave & 0.02 & 0.3 & 0.52 & 0.26 & 0 & 1 \\
Convex & 0 & 0 & 0 & 0.24 & 0.01 & 0 \\
Log & 0 & 0 & 0 & 0 & 0 & 0 \\
Barker & 0.34 & 0.7 & 0.96 & 0.98 & 1 & 0 \\
Zad-Ch & 0 & 0.02 & 0.21 & 0.13 & 0.07 & 0 \\
Frank & 0 & 0 & 0 & 0.06 & 1 & 1 \\
P1 & 0 & 0.03 & 0 & 0 & 0 & 0 \\
P2 & 0 & 0.06 & 0.8 & 0.75 & 0.01 & 0 \\
P3 & 0 & 0 & 0 & 0 & 0 & 0 \\
P4 & 0 & 0 & 0 & 0 & 0 & 0 \\
Px & 0 & 0.03 & 0 & 0 & 0 & 0 \\
Overall & 0.08 & 0.18 & 0.34 & 0.34 & 0.32 & 0.31 \\
\hline
\end{tabular}


is unexpected, as it was hypothesized that the variations would change the shape enough to degrade results. Appendix C.7 contains the detailed confusion matrices at $6 \mathrm{~dB}$ SNR, which details the similarity between results to a modulation level at different carrier frequencies.

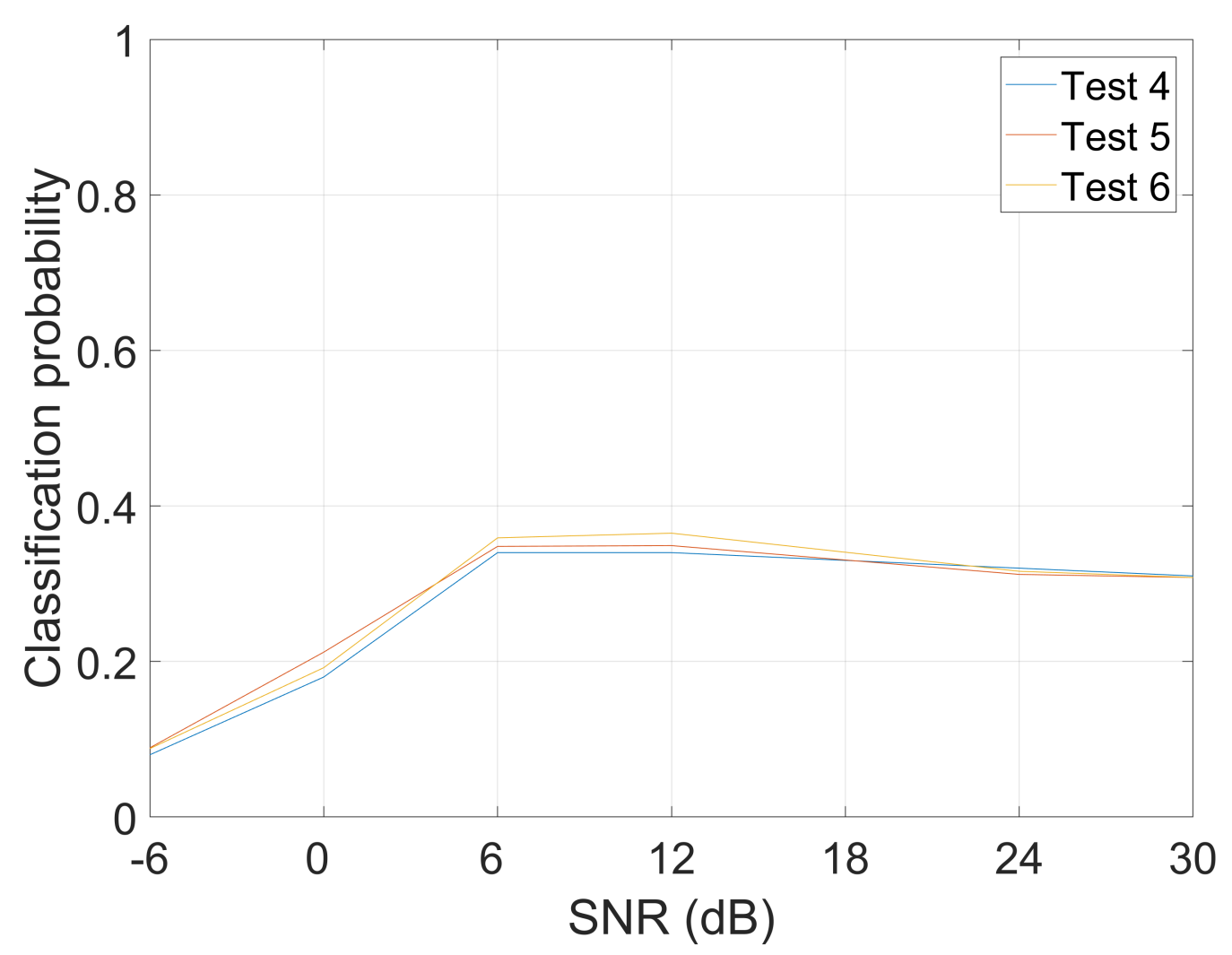

Figure 20: ANN Performance with Carrier Frequency Variation

\subsection{CNN Classification}

Effective CNN training requires 1000's of unique data samples. Using the radar signal generation software, in combination with random AGWN, almost 10000 samples per data set were produced. The subpulse parameters are listed in table 3. Training was performed at a variety of SNRs, with the lowest being $12 \mathrm{~dB}$. Other test 
sets range from -6 to $30 \mathrm{~dB}$, with various subpulse parameters.

Overall classifier performance for each test set is displayed in fig. 21, while an individual breakdown for each test is presented in tables 8 to 10 .

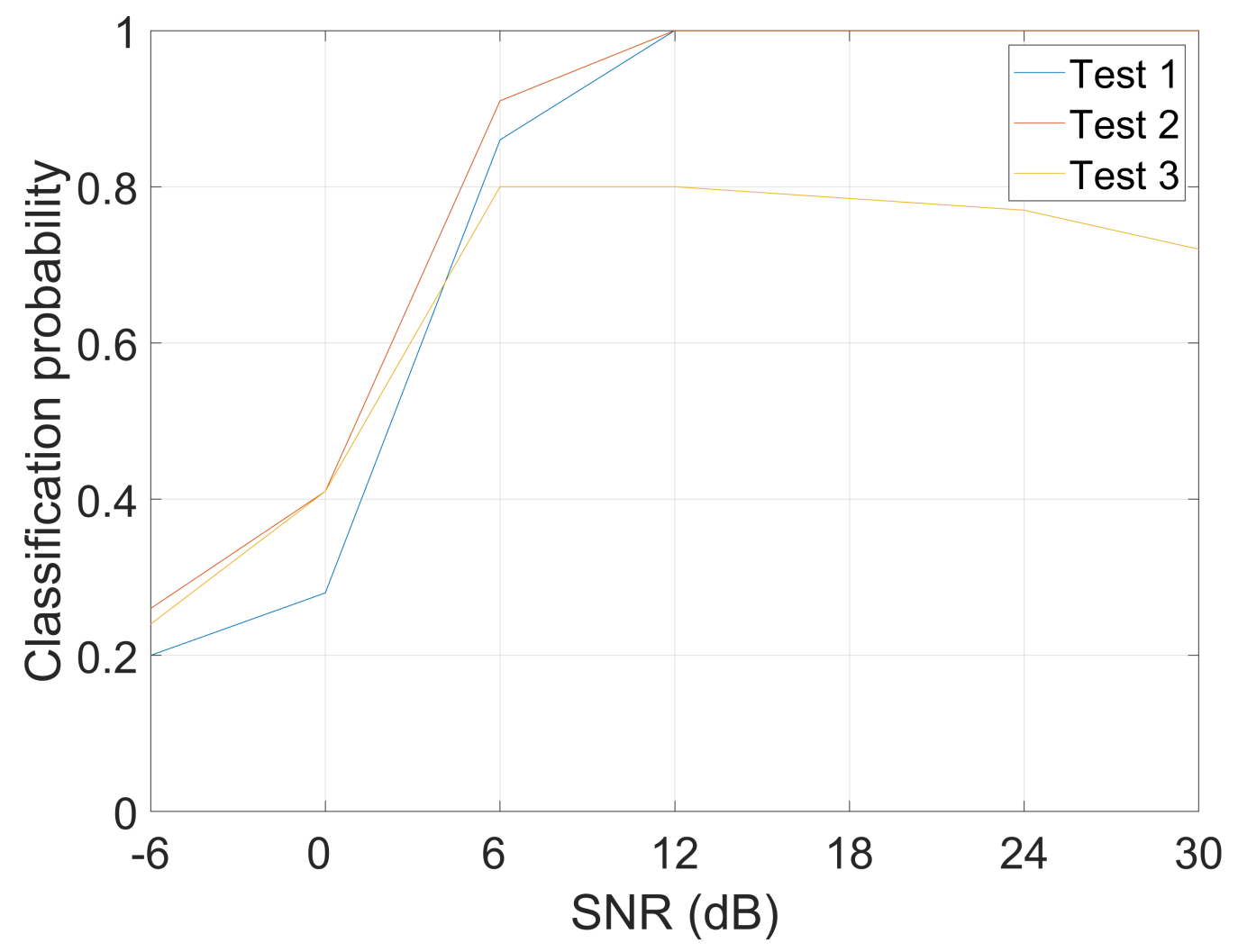

Figure 21: Overall CNN Classification Performance

Tests 1 and 2 have similar parameters as the training set. This is designed to give a estimation of performance when the signal environment is similar to the expected environment. At high SNR, the performance is perfect. This is due to the classifier being trained in this region. Training the classifier in the low SNR region resulted in lower performance overall, as high amounts of noise impacted the convergence of the training algorithm. In the lower SNR region $(<6 \mathrm{~dB})$, the classifier performance was degraded specifically for PSK waveforms. Examining the confusion matrices in 
Table 8: CNN Classifier Performance for Test 1

\begin{tabular}{ccccccc}
\hline MOP & $\mathbf{- 6} \mathbf{d B}$ & $\mathbf{0} \mathbf{d B}$ & $\mathbf{6} \mathbf{d B}$ & $\mathbf{1 2} \mathbf{d B}$ & $\mathbf{2 4} \mathbf{d B}$ & $\mathbf{3 0} \mathbf{d B}$ \\
None & 1 & 1 & 1 & 1 & 1 & 1 \\
LFM & 0 & 0 & 0.90 & 1 & 1 & 1 \\
Concave & 0 & 0 & 0.98 & 1 & 1 & 1 \\
Convex & 0.79 & 0.91 & 1 & 1 & 1 & 1 \\
Log & 0.83 & 0.95 & 0.99 & 1 & 1 & 1 \\
Barker & 0.02 & 0.65 & 1 & 1 & 1 & 1 \\
Zad-Ch & 0 & 0 & 0.07 & 1 & 1 & 1 \\
Frank & 0 & 0.13 & 1 & 1 & 1 & 1 \\
P1 & 0 & 0 & 1 & 1 & 1 & 1 \\
P2 & 0 & 0.02 & 0.91 & 1 & 1 & 1 \\
P3 & 0 & 0 & 0.62 & 1 & 1 & 1 \\
P4 & 0 & 0 & 0.80 & 1 & 1 & 1 \\
Px & 0 & 0 & 0.89 & 1 & 1 & 1 \\
Overall & 0.20 & 0.28 & 0.86 & 1 & 1 & 1 \\
\hline
\end{tabular}

Appendices C.4 to C.5, it is noted that much of the incorrect classifications are among the various PSK waveforms. For example in fig. 51, the Zadoff-Chu modulation is almost entirely incorrectly classified as Frank. It is similar in other scenarios, often with many modulations incorrectly classified to a specific waveform. This leads to a clustering phenomenon, which can still be useful. While the overall classification accuracy may degrade at lower SNRs, the system can still group like modulations.

Test 3 tests a more realistic scenario with different subpulse parameters for each MOP, often outside the training range. Other general simulation parameters are kept the same as in table 1 . The results are detailed in table 10, with detailed confusion matrices found in appendix C. At high SNRs $(>6 \mathrm{~dB})$ the classification performance 
Table 9: CNN Classifier Performance for Test 2

\begin{tabular}{ccccccc}
\hline MOP & $-\mathbf{6} \mathrm{dB}$ & $\mathbf{0} \mathrm{dB}$ & $\mathbf{6} \mathrm{dB}$ & $\mathbf{1 2} \mathrm{dB}$ & $\mathbf{2 4} \mathrm{dB}$ & $\mathbf{3 0} \mathrm{dB}$ \\
None & 1 & 1 & 1 & 1 & 1 & 1 \\
LFM & 0 & 0.01 & 0.99 & 1 & 1 & 1 \\
Concave & 0 & 0 & 1 & 1 & 1 & 1 \\
Convex & 0.80 & 1 & 1 & 1 & 1 & 1 \\
Log & 0.95 & 1 & 1 & 1 & 1 & 1 \\
Barker & 0.44 & 0.93 & 1 & 1 & 1 & 1 \\
Zad-Ch & 0 & 0.21 & 1 & 1 & 1 & 1 \\
Frank & 0.18 & 0.88 & 1 & 1 & 1 & 1 \\
P1 & 0 & 0.06 & 0.94 & 1 & 1 & 1 \\
P2 & 0 & 0.11 & 1 & 1 & 1 & 1 \\
P3 & 0 & 0.02 & 0.55 & 1 & 1 & 1 \\
P4 & 0 & 0.08 & 0.51 & 1 & 1 & 1 \\
Px & 0 & 0.02 & 0.77 & 1 & 1 & 1 \\
Overall & 0.26 & 0.41 & 0.91 & 1 & 1 & 1 \\
\hline
\end{tabular}


Table 10: CNN Classifier Performance for Test 3

\begin{tabular}{ccccccc}
\hline MOP & $\mathbf{- 6} \mathbf{d B}$ & $\mathbf{0} \mathbf{d B}$ & $\mathbf{6} \mathbf{d B}$ & $\mathbf{1 2} \mathbf{d B}$ & $\mathbf{2 4} \mathbf{d B}$ & $\mathbf{3 0} \mathbf{d B}$ \\
None & 0.99 & 1 & 1 & 1 & 1 & 1 \\
LFM & 0 & 0 & 0.98 & 1 & 1 & 1 \\
Concave & 0 & 0.06 & 1 & 1 & 1 & 1 \\
Convex & 0.50 & 0.95 & 1 & 1 & 1 & 1 \\
Log & 0.35 & 0.63 & 0.99 & 1 & 1 & 1 \\
Barker & 0.80 & 1 & 1 & 1 & 1 & 1 \\
Zad-Ch & 0 & 0.22 & 0.58 & 0.71 & 0.87 & 0.31 \\
Frank & 0.51 & 1 & 1 & 0.63 & 0.18 & 1 \\
P1 & 0 & 0.33 & 0.96 & 1 & 1 & 1 \\
P2 & 0 & 0.05 & 0.95 & 1 & 1 & 0 \\
P3 & 0 & 0.04 & 0.91 & 1 & 1 & 1 \\
P4 & 0 & 0 & 0 & 0 & 0 & 0 \\
Px & 0 & 0 & 0 & 0 & 0 & 0 \\
Overall & 0.24 & 0.41 & 0.80 & 0.80 & 0.77 & 0.72 \\
\hline
\end{tabular}

is approximately $80 \%$, with degradation at low SNRs similar to the previous tests. This suggests that a classifiers performance is highly correlated with the training data. Combining sufficient computing power with the ability of the radar signal generator to generate a limitless variety of artificial waveforms, a very high performance classifier could be trained. Additionally, while examining the confusion matrices it is apparent that the major classification confusion only occurs among PSK pulses. For example, at $6 \mathrm{~dB}$ (fig. 57) the CNN has almost no errors for FM pulses, with most of the confusion occurring for the P4 and Px modulations.

As per the ANN network, the center frequency variation did not significantly affect results for the CNN. Examining fig. 22, the performance is approximately the same at lower carrier frequencies. The classifier confusion on individual pulses changed, but 
overall the accuracy was similar. As stated previously, these results are surprising considering the networks are trained on a static carrier frequency. See appendix C.7 for a sample of the confusion matrices for the carrier frequency variation.

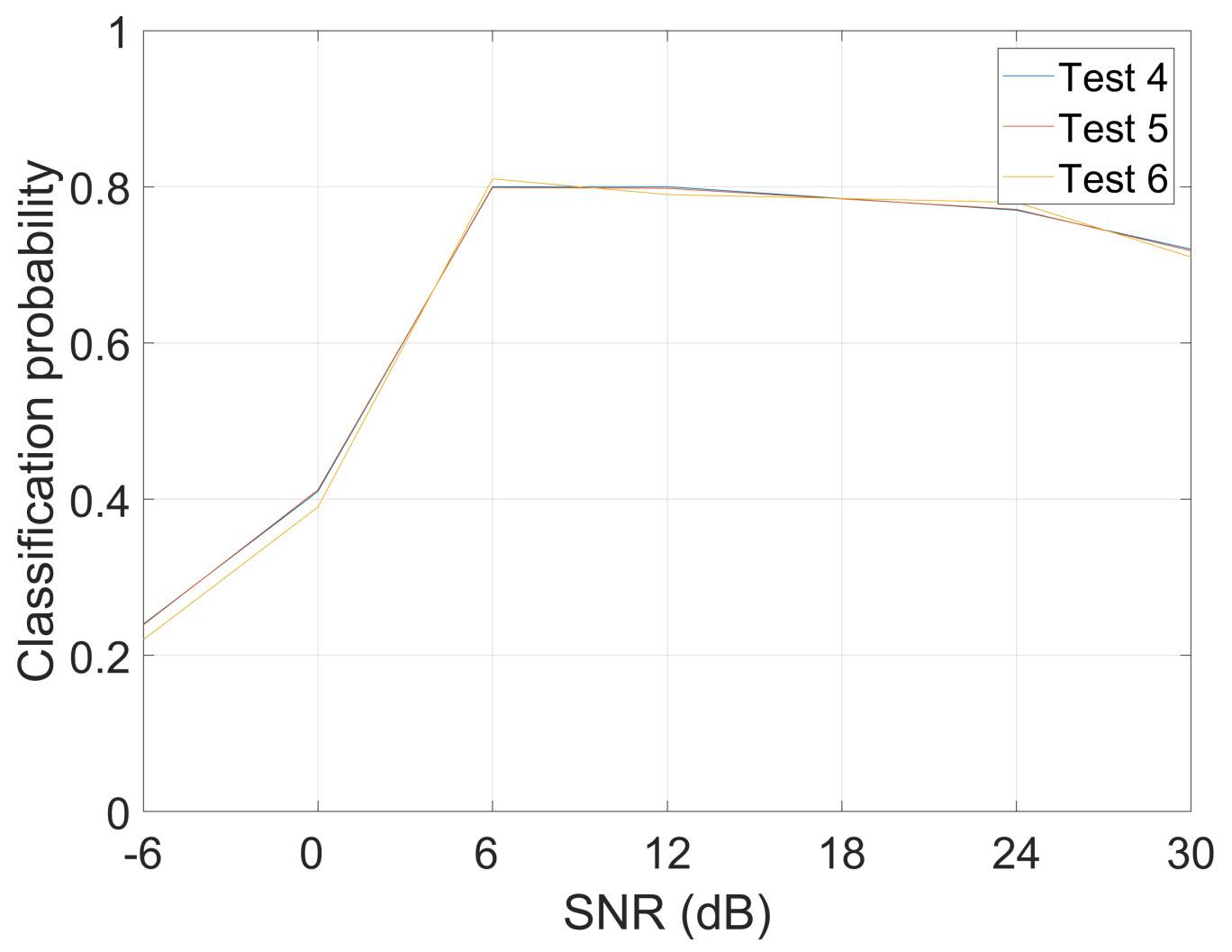

Figure 22: CNN Performance with Carrier Frequency Variation

\subsection{Classifier Comparison}

The simplest method is the HDT/ANN hybrid. This solution can classify pulses correctly under ideal conditions, but has very poor performance in a real signal environment. The standout result from this test is the results for unmodulated waveforms. At SNRs greater than $0 \mathrm{~dB}$ the classification is almost perfect. When examining the 
Table 11: Average Latency Per Pulse

\begin{tabular}{cccc}
\hline Transform Type & HDT / ANN (ms) & ANN (ms) & CNN (ms) \\
PWVD & 58.73 & 56.42 & 76.66 \\
CW & 274.48 & 272.18 & 292.42 \\
Ignored & 2.36 & 0.060 & 20.30 \\
\hline
\end{tabular}

classifier confusion in appendix $\mathrm{C}$, it is noted that the ANN and CNN misclassifications are often the result of classifying a MOP as unmodulated. Further implementation could eliminate errors by using the HDT method for unmodulated pulses to eliminate this source of error from the ANN/CNN methods.

Examining table 11, the HDT/ANN was not the fastest method. This is a factor of the unoptimized frequency transforms and split of pulses. With more frequency modulated pulses, the system could perform faster as the TF transform component has the highest latency. The PWVD and CW transforms had latency's of $56.36 \mathrm{~ms}$ and $272.12 \mathrm{~ms}$ per pulse respectively. Ignoring the transforms, the HDT architecture itself has a latency of $2.36 \mathrm{~ms}$ per pulse. Note that these tests were not performed in a parallel environment, which would speed up processing significantly.

The ANN classifier has marginally better performance when utilizing the CW transformation. This transformation produces excellent TF results but is very computationally expensive when compared with the PWVD transformation. The PWVD transform has comparable performance for the ANN classifier, and is preferred for large data sets. This method did not generalize well. The ANN has the lowest architecture latency, with classification taking approximately $0.06 \mathrm{~ms}$ per pulse. This is due to the simplicity of MLP networks, with pulses analyzed quickly due to the small number of layers and neurons.

The CNN classifier produced the best performance of all three methods. This 
proved to be true even with the utilization of the PWVD transform, and could efficiently examine waveforms in approximately $20.30 \mathrm{~ms}$ per pulse. While being the slowest method in respect to latency, the classification accuracy was the best. The number and size of layers is the main factor in the network latency. With more optimization and redesign of the network, more computational power, the real-time classification of large amounts of waveforms is a possibility. Additionally using an efficient language such as Python or $\mathrm{C}++$ will help reduce latency.

It was noted that changes in the carrier frequency did not significantly effect results. Testing in a real EM environment is necessary to confirm this. A source of error in these results is that the sampling frequency are kept constant. If a higher sampling frequency was used and the pulse downsampled, the classification results could be affected. It was assumed that in a full system the pre-processing would negate these effects, but this was never verified. 


\section{Chapter 6}

\section{Conclusion}

In this thesis, three classification systems for MOP discrimination of thirteen waveforms has been developed and tested. The classifiers were evaluated and compared based on their performance accuracy and applicability to a real-time realistic EM environment. Both positive and negative characteristics for each system has been discussed, with recommendations for improvement given where warranted.

The HDT/ANN hybrid produced good results when in a known, non-noisy signal environment. When this method was tested in a noisy, realistic situation the classification performance was close to zero. The upside of the HDT/ANN was the low computational complexity, and the results in classifying unmodulated pulses. The feature based ANN had an acceptable classification accuracy when pulses were at a high SNR. However, in a generalized environment this method proved to be ineffective. This method is acceptable in a predictable environment which has readily available training data. The CNN classifier proved to be the most effective in classification performance. Even when tested with signals that were different from the training set, the performance was much higher than other methods. The latency for the ANN system was the lowest, with the HDT/ANN hybrid in a comparable range. It was noted that carrier frequency variation did not significantly affect results. 
The topic of MOP discrimination will continue to be heavily researched, as an increasingly congested EM spectrum necessitates automation of modulation classification. The offline AMC solutions proposed in this thesis could potentially be modified to fit an online learning approach, which is the next step in intelligent signal classification.

\subsection{Overview of Contributions}

This thesis introduced three solutions to the AMC problem. The first proposed design is a combined machine learning approach, utilizing Artificial Neural Networks and Hierarchical Design Trees. The second is an ANN based on feature extraction techniques. The third is a CNN, which utilizes TF transformations in the form of color images. The proposed solutions were examined and validated through testing and simulation, along with the challenges inherent to the AMC problem. Simulations were run with a variety of pulse parameters, with a full system latency analysis performed. The MOP solutions can be implemented and deployed on embedded field platforms. The results had a classification accuracy comparable to most systems in literature.

\subsection{Future Work}

The solutions in this work only consider the intentional MOP. A similar technique could be used to classify the UMOP to differentiate between individual emitters. Online learning implementation would be extremely valuable to classify new and exotic MOPs. When a pulse triggers a specific uncertainty threshold, the system could learn a new ANN model based on the new pulse identified. This would enable 
automatic learning of new pulse types.

There are three recommendations for future testing and validation of the system. Firstly, expanding the scope to include other types of modulation would be valuable for expanding the system and making it useful outside of academia. These other modulation types include frequency shift keyed, such as Costa code, and continuous wave radar. Secondly, further simulations using measured field pulse data would be valuable to prove both the RSG software and the MOP discrimination technique. Thirdly, the testing of the CNN method could be exapanded greatly through training on very large datasets containing both simulated and field data. With appropriate computational hardware, a CNN model could be developed that would produce very impressive results. 


\section{List of References}

[1] M. Skolnik, Radar Handbook, 1990.

[2] R. G. Wiley, ELINT: The interception and analysis of radar signals. Artech House, 2006.

[3] N. Levanon and E. Mozeson, Radar Signals, ser. Wiley - IEEE. Wiley, 2004.

[4] M. Richards, Fundamentals of Radar Signal Processing, ser. Professional Engineering. McGraw-Hill Education, 2005.

[5] J. Lunden, L. Terho, and V. Koivunen, "Classifying pulse compression radar waveforms using time frequency distributions," in Proc. 39th Annual Conf. Information Sciences and Systems (CISS 2005). Citeseer, 2005.

[6] P. B. Rapajic and R. A. Kennedy, "Merit factor based comparison of new polyphase sequences," IEEE Communications Letters, vol. 2, no. 10, pp. 269270, Oct 1998.

[7] A. Abdelmutalab, K. Assaleh, and M. El-Tarhuni, "Automatic modulation classification using polynomial classifiers," in 2014 IEEE 25th Annual International Symposium on Personal, Indoor, and Mobile Radio Communication (PIMRC), Sept 2014, pp. 806-810.

[8] O. A. Dobre, A. Abdi, Y. Bar-Ness, and W. Su, "Survey of automatic modulation classification techniques: classical approaches and new trends," IET Communications, vol. 1, no. 2, pp. 137-156, April 2007.

[9] D. Kutman, "Solutions for radar pulse deinterleaving," Ph.D. dissertation, Carleton University, 2011.

[10] A. E. Abdelmutalab, "Learning-based automatic modulation classification," Ph.D. dissertation, 2015.

[11] S. Theodoridis and K. Koutroumbas, Pattern recognition, 4th ed. Burlington, MA: Academic Press, 2009;2008;.

[12] I. Goodfellow, Y. Bengio, and A. Courville, Deep Learning. MIT Press, 2016.

[13] C. Christodoulou and M. Georgiopoulos, Applications of neural networks in electromagnetics. Artech House, Inc., 2000.

[14] C. M. Bishop, Neural networks for pattern recognition. Oxford university press, 1995. 
[15] D. Ciregan, U. Meier, and J. Schmidhuber, "Multi-column deep neural networks for image classification," in Computer vision and pattern recognition (CVPR), 2012 IEEE conference on. IEEE, 2012, pp. 3642-3649.

[16] A. Krizhevsky, I. Sutskever, and G. E. Hinton, "Imagenet classification with deep convolutional neural networks," in Advances in neural information processing systems, 2012, pp. 1097-1105.

[17] I. Goodfellow, Y. Bengio, A. Courville, and Y. Bengio, Deep learning. MIT press Cambridge, 2016, vol. 1.

[18] J. Lundén and V. Koivunen, "Automatic radar waveform recognition," IEEE Journal of Selected Topics in Signal Processing, vol. 1, no. 1, pp. 124-136, 2007.

[19] S. H. Kong, M. Kim, and L. M. Hoang, "Automatic lpi radar waveform recognition using cnn," IEEE Access, vol. PP, no. 99, pp. 1-1, 2018.

[20] J. Ming, H. Guobing et al., "Intrapulse modulation recognition of radar signals based on statistical tests of the time-frequency curve," in Electronics and Optoelectronics (ICEOE), 2011 International Conference on, vol. 1. IEEE, 2011, pp. V1-300.

[21] V. M. Subbarao, S. N. Khasim, and J. Thati, "A novel technique for automatic modulation classification and time-frequency analysis of digitally modulated signals," 2015.

[22] T. R. Kishore and K. D. Rao, "Automatic intrapulse modulation classification of advanced lpi radar waveforms," IEEE Transactions on Aerospace and Electronic Systems, vol. 53, no. 2, pp. 901-914, April 2017.

[23] P. K. HL and L. Shrinivasan, "Automatic digital modulation recognition using minimum feature extraction," in Computing for Sustainable Global Development (INDIACom), 2015 2nd International Conference on. IEEE, 2015, pp. 772-775.

[24] H. Jiang, Z. Pang, P. Tang, and L. Jia, "Intrapulse modulation recognition based on pulse description words," in Image and Signal Processing (CISP), 2013 6th International Congress on, vol. 3. IEEE, 2013, pp. 1367-1371.

[25] B. Park and J. M. Ahn, "Intra-pulse modulation recognition using pulse description words and complex waveforms," in 2017 International Conference on Information and Communication Technology Convergence (ICTC), Oct 2017, pp. $555-560$.

[26] V. Iglesias, J. Grajal, P. Royer, M. A. Sanchez, M. Lopez-Vallejo, and O. A. Yeste-ojeda, "Real-time low-complexity automatic modulation classifier for pulsed radar signals," IEEE Transactions on Aerospace and Electronic Systems, vol. 51, no. 1, pp. 108-126, January 2015.

[27] J. L. Xu, W. Su, and M. Zhou, "Likelihood-ratio approaches to automatic modulation classification," IEEE Transactions on Systems, Man, and Cybernetics, Part C (Applications and Reviews), vol. 41, no. 4, pp. 455-469, July 2011. 
[28] S. L. Marple, "Computing the discrete-time 'analytic' signal via fft," in Conference Record of the Thirty-First Asilomar Conference on Signals, Systems and Computers (Cat. No.97CB36136), vol. 2, 1997, pp. 1322-1325 vol.2.

[29] H. Xiao, Y. Q. Shi, W. Su, and J. A. Kosinski, "Automatic classification of analog modulation schemes," in Radio and Wireless Symposium (RWS), 2012 IEEE. IEEE, 2012, pp. 5-8.

[30] M. Sun, C. C. Li, L. N. Sekhar, and R. J. Sclabassi, "Efficient computation of the discrete pseudo-wigner distribution," IEEE Transactions on Acoustics, Speech, and Signal Processing, vol. 37, no. 11, pp. 1735-1742, Nov 1989.

[31] H.-I. Choi and W. J. Williams, "Improved time-frequency representation of multicomponent signals using exponential kernels," IEEE Transactions on Acoustics, Speech, and Signal Processing, vol. 37, no. 6, pp. 862-871, 1989.

[32] R. Gonzalez and R. Woods, Digital Image Processing. Addison-Wesley, 1992.

[33] C. H. Teh and R. T. Chin, "On image analysis by the methods of moments," IEEE Transactions on Pattern Analysis and Machine Intelligence, vol. 10, no. 4, pp. 496-513, Jul 1988.

[34] C. University, "Time-frequency toolbox," http://tftb.nongnu.org/, accessed: 2018-03-28.

[35] U. C. C. John M. O' Toole, "Fast and memory efficient algorithms for quadratic time frequency distributions," http://otoolej.github.io/code/memeff_TFDs/, accessed: 2018-04-10.

[36] M. F. Møller, "A scaled conjugate gradient algorithm for fast supervised learning," Neural networks, vol. 6, no. 4, pp. 525-533, 1993. 
Appendix A

\section{MATLAB Generated Waveforms}

\section{A.1 Received Modulations}

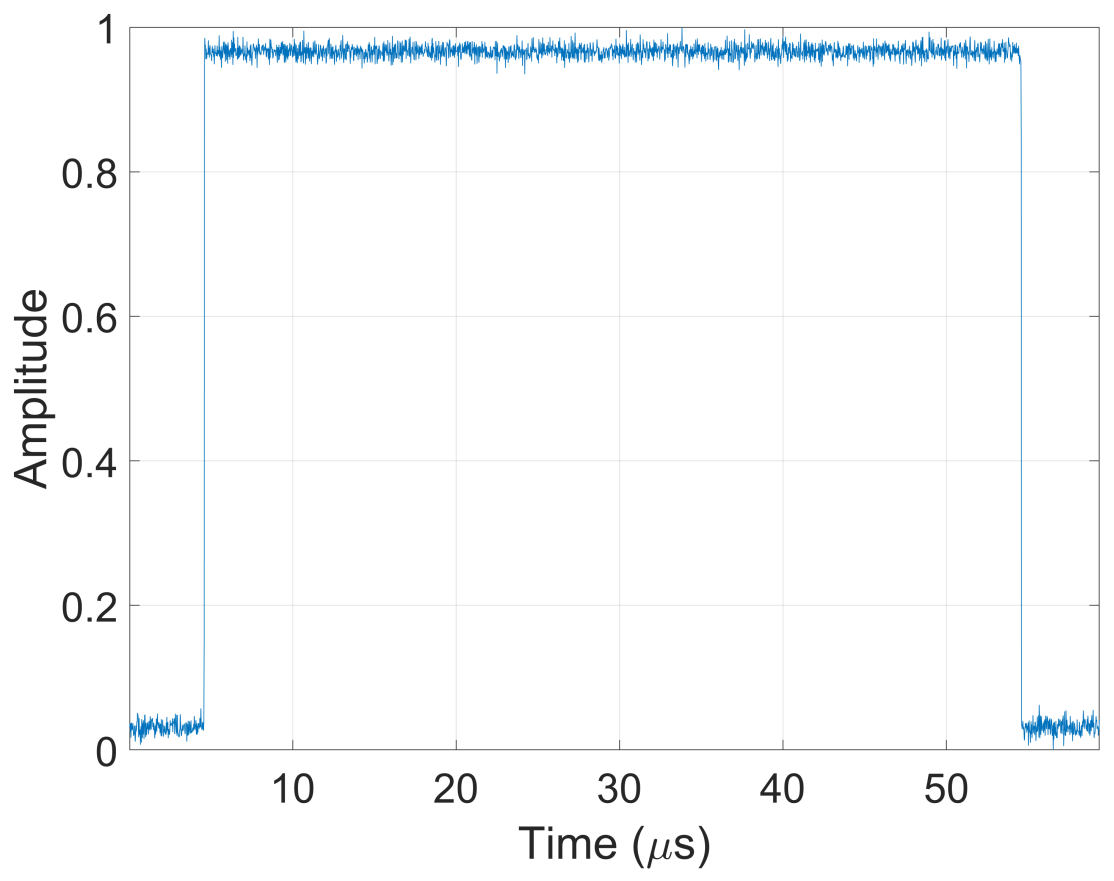

Figure 23: Unmodulated received pulse generated in MATLAB 


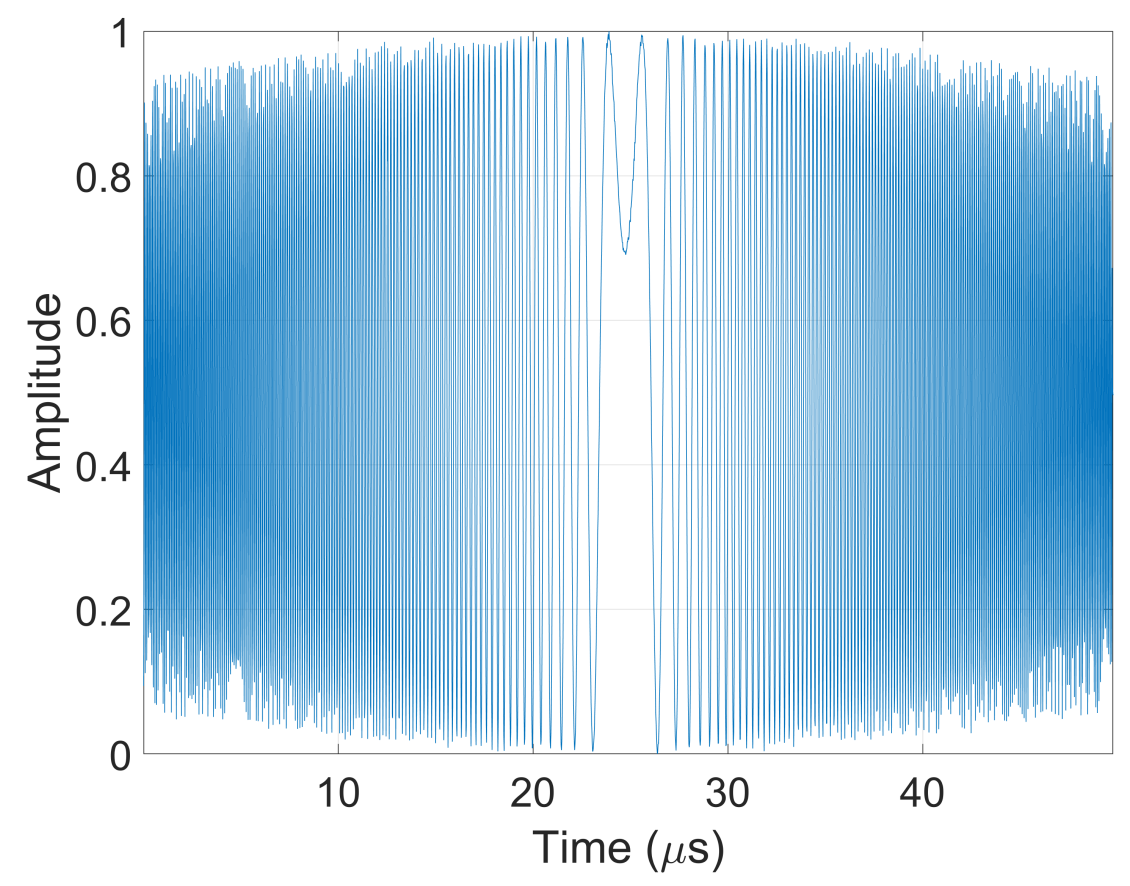

Figure 24: LFM received pulse generated in MATLAB

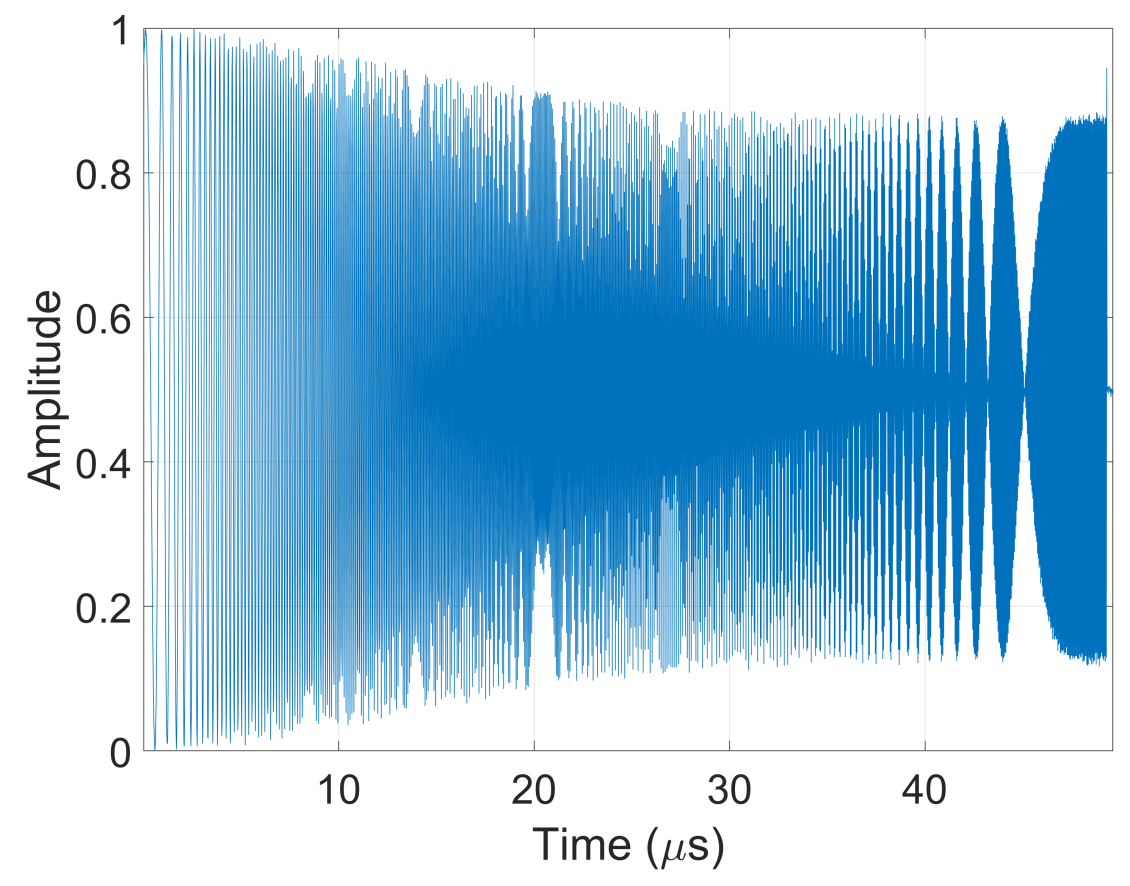

Figure 25: NLFM Concave received pulse generated in MATLAB 


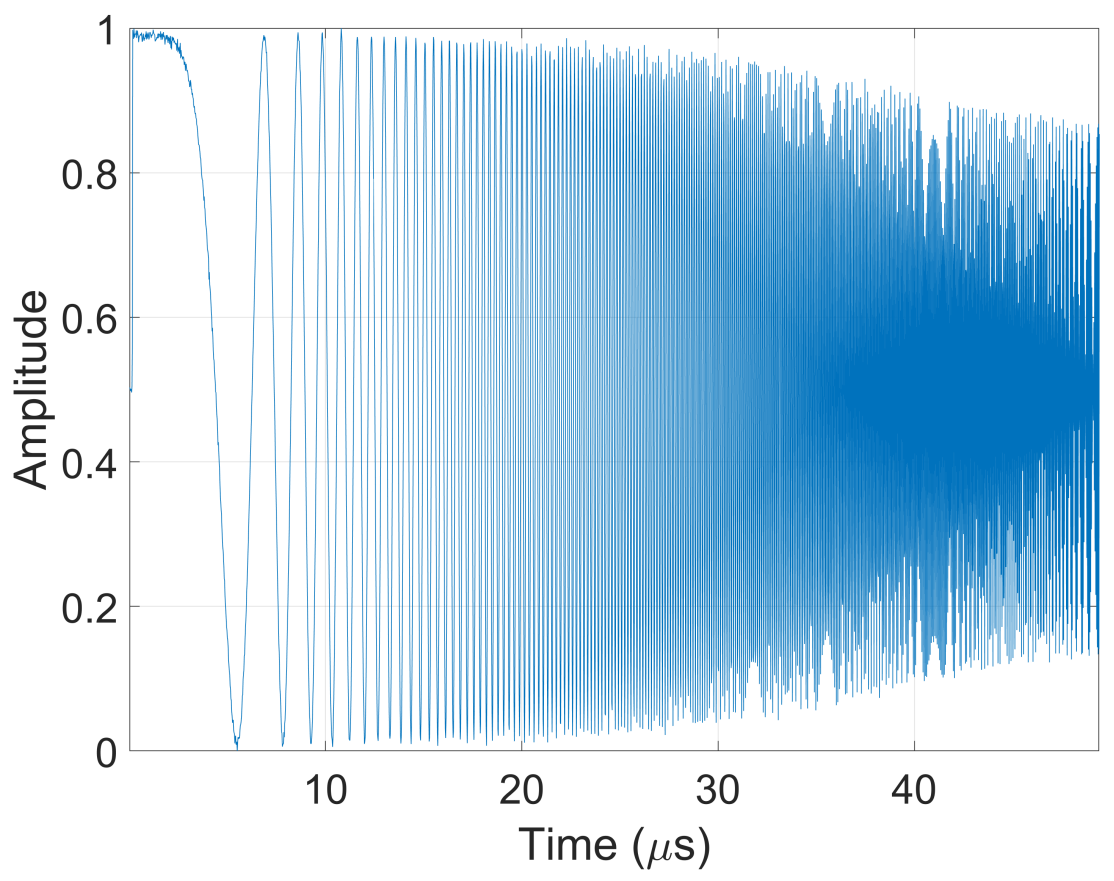

Figure 26: NLFM Convex received pulse generated in MATLAB

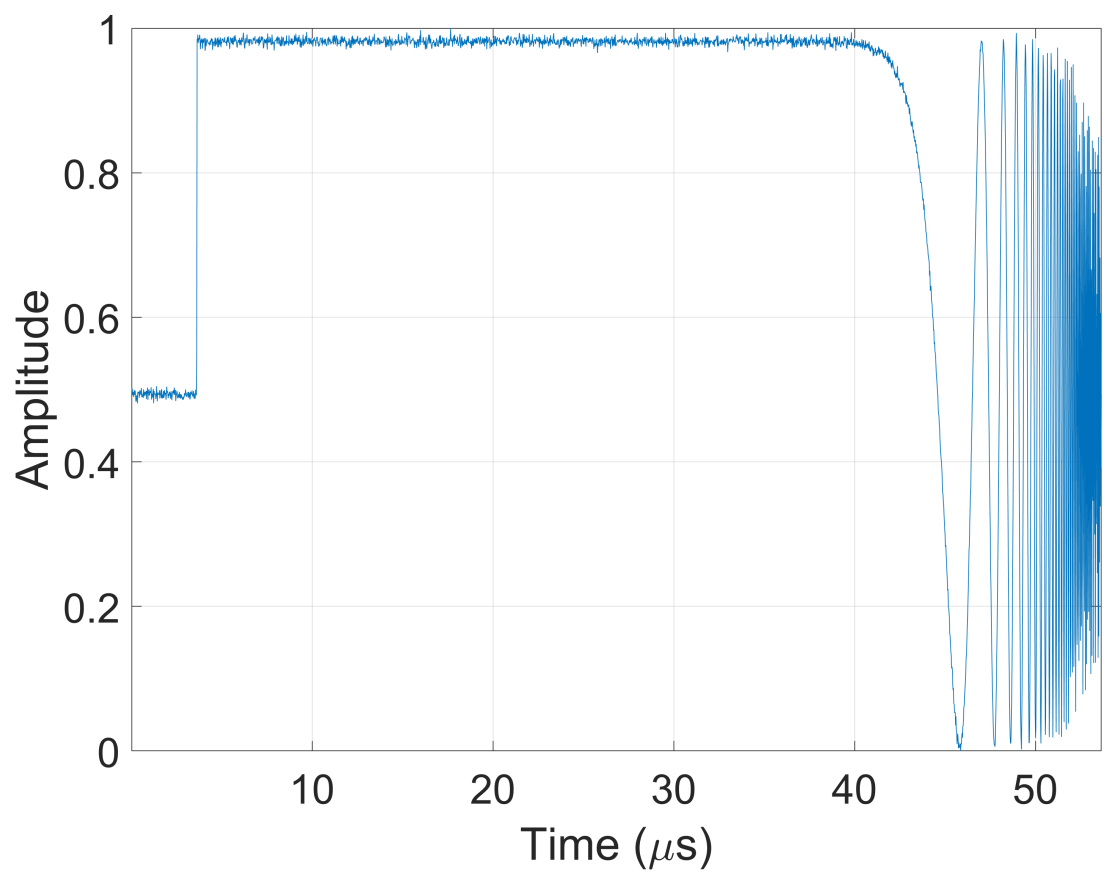

Figure 27: NLFM logarithmic received pulse generated in MATLAB 


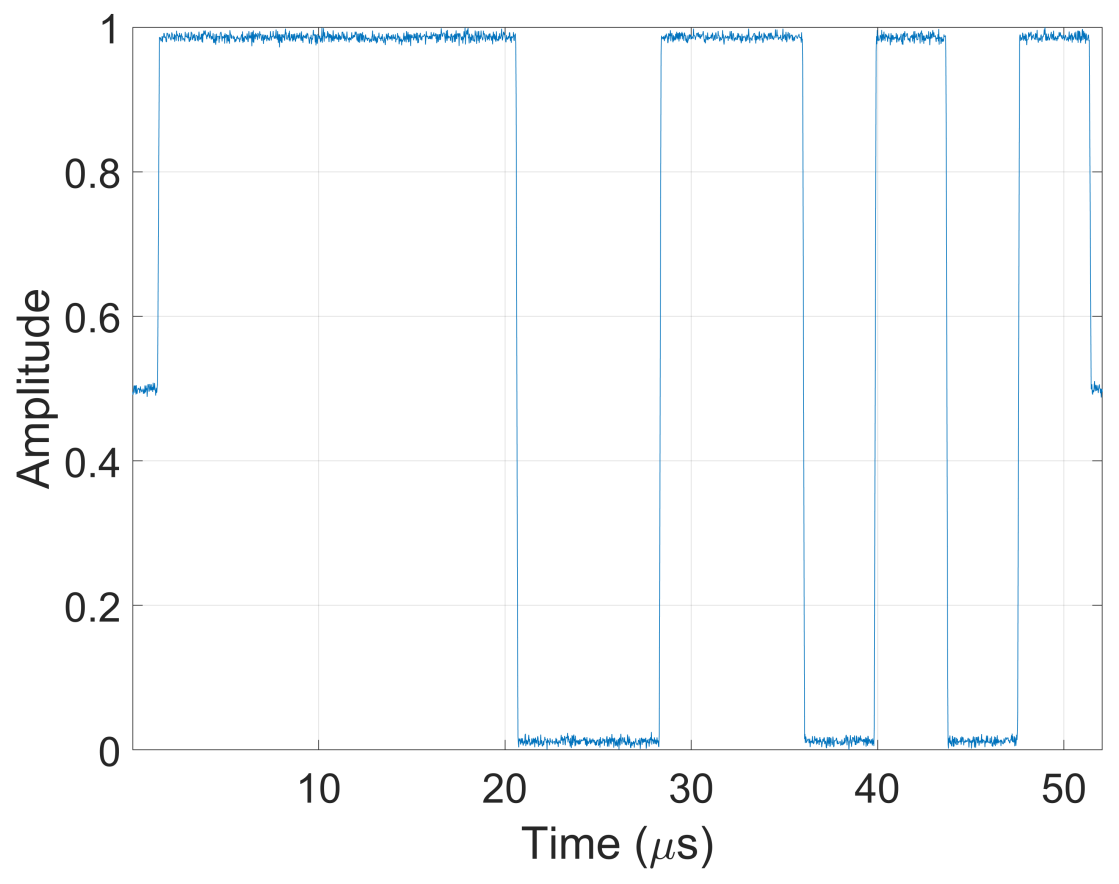

Figure 28: Barker 13 received pulse generated in MATLAB

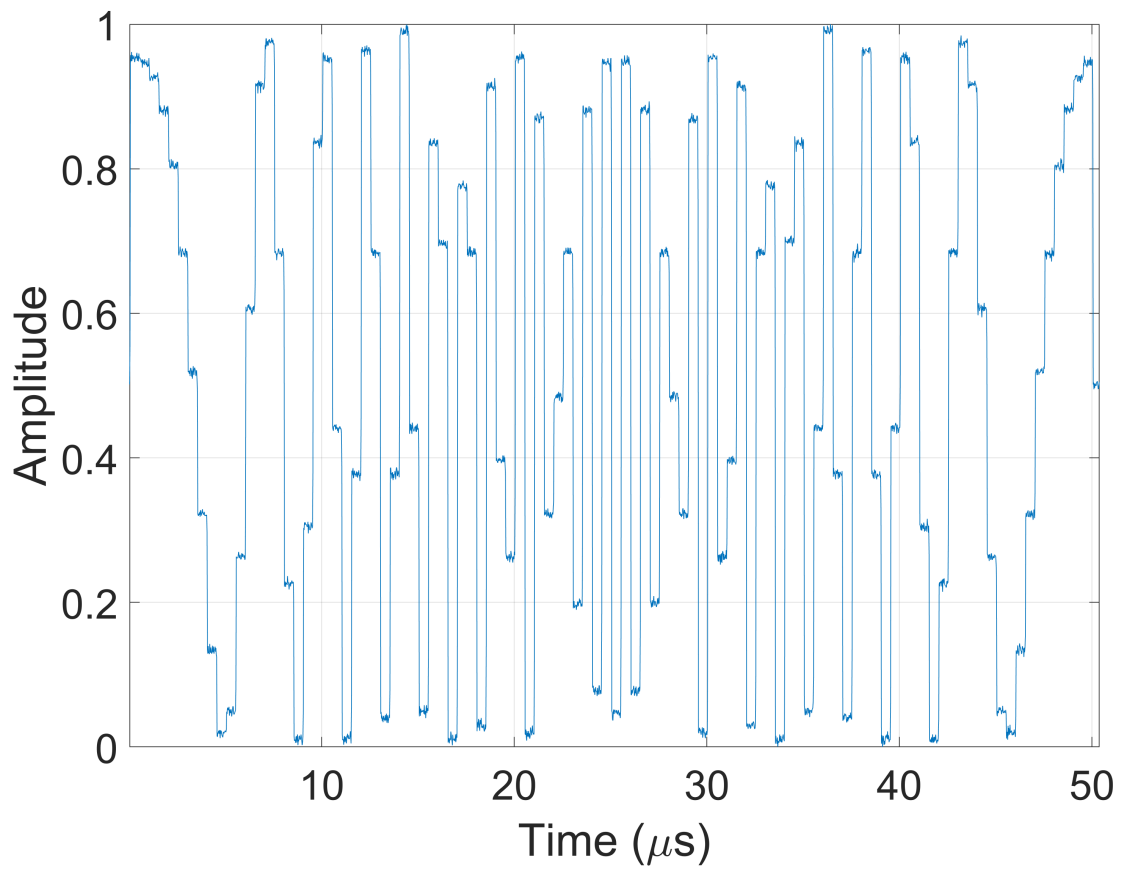

Figure 29: Zadoff-Chu received pulse generated in MATLAB 


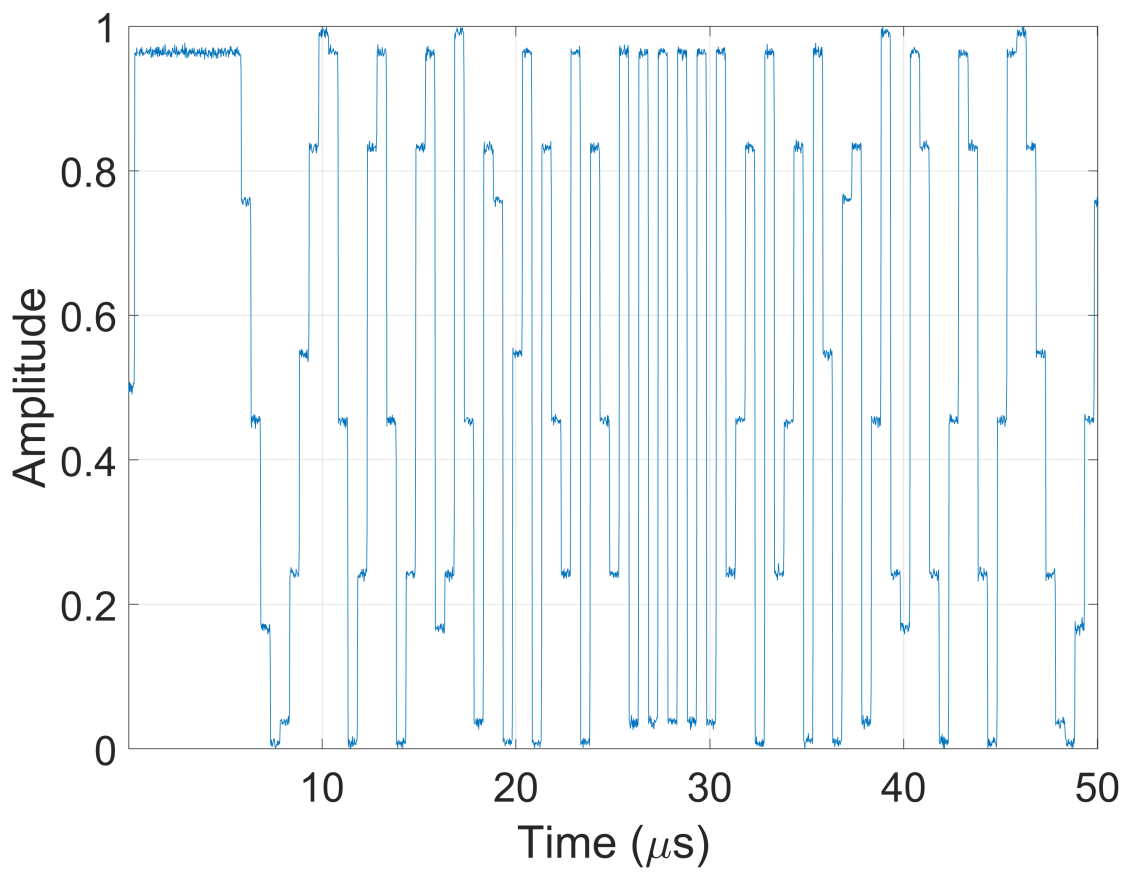

Figure 30: Frank received pulse generated in MATLAB

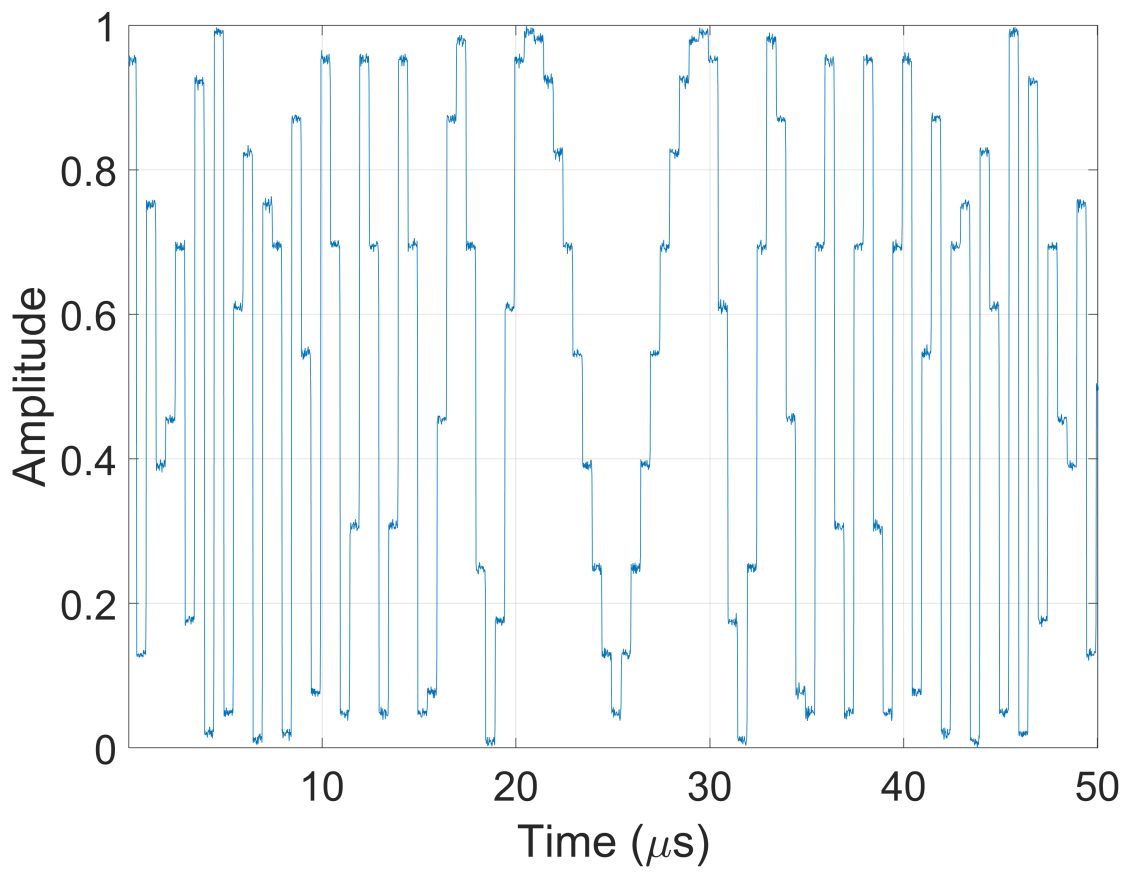

Figure 31: $\mathrm{P} 1$ received pulse generated in MATLAB 


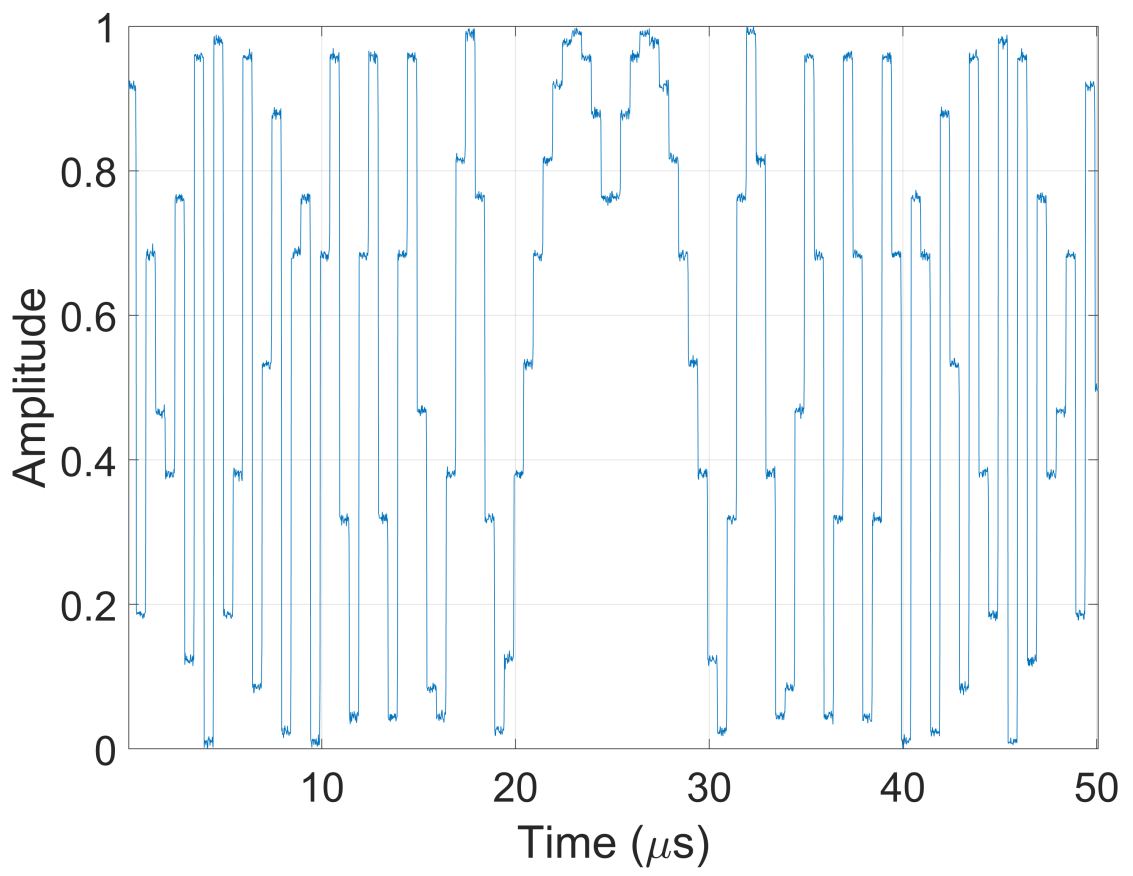

Figure 32: $P 2$ received pulse generated in MATLAB

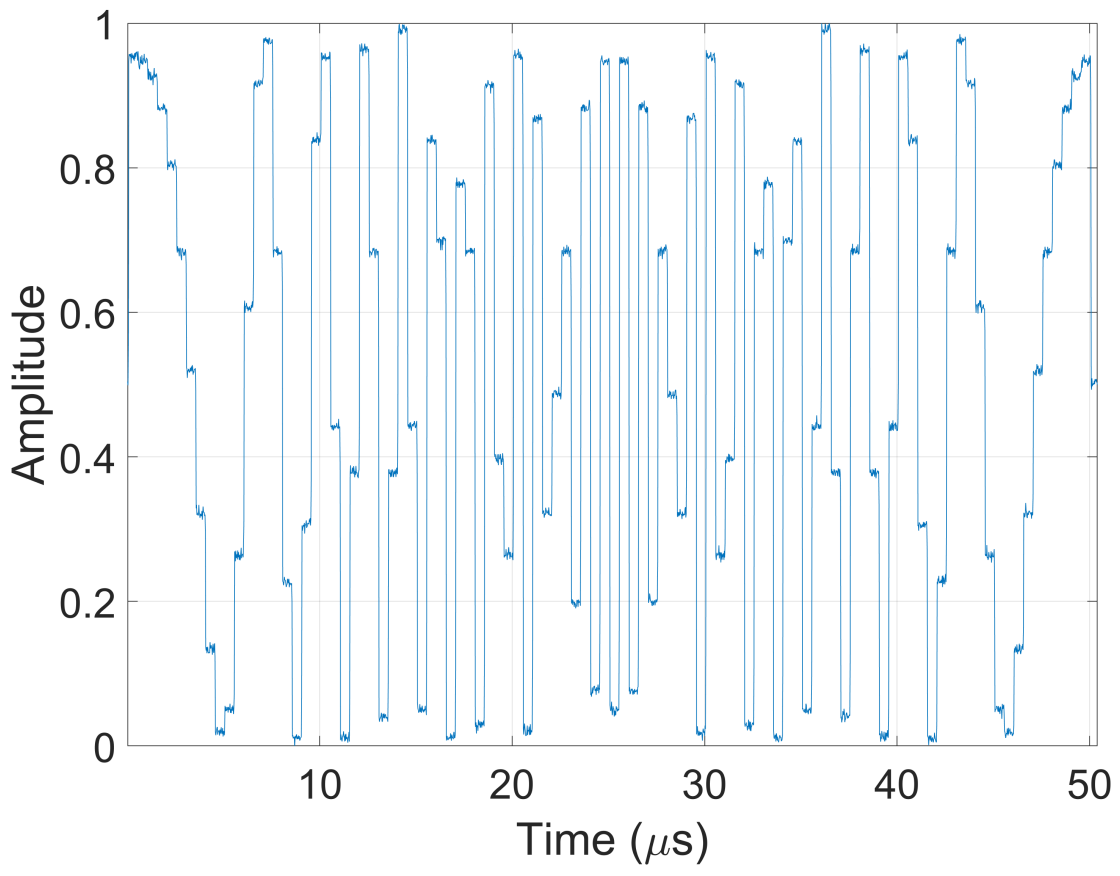

Figure 33: $P 3$ received pulse generated in MATLAB 


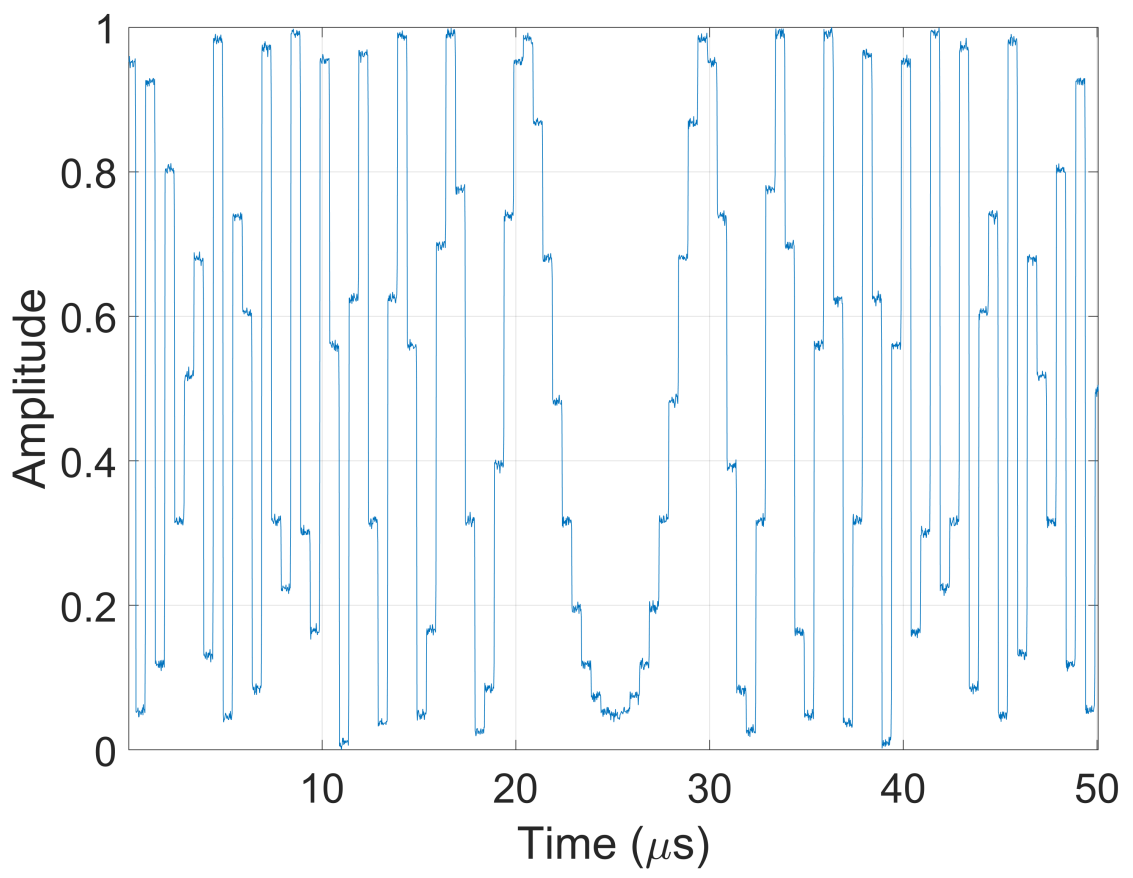

Figure 34: $\mathrm{P} 4$ received pulse generated in MATLAB

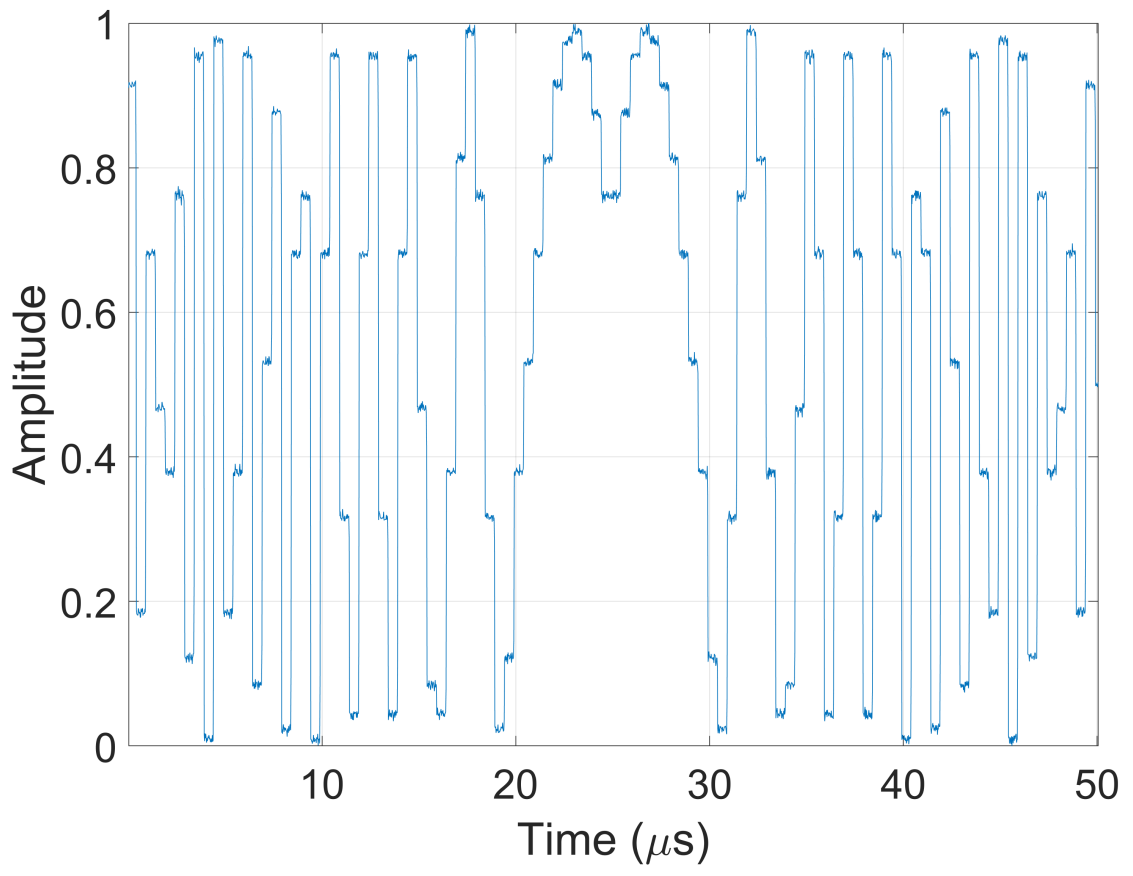

Figure 35: Px received pulse generated in MATLAB 


\section{A.2 Other Transmitted Modulations}

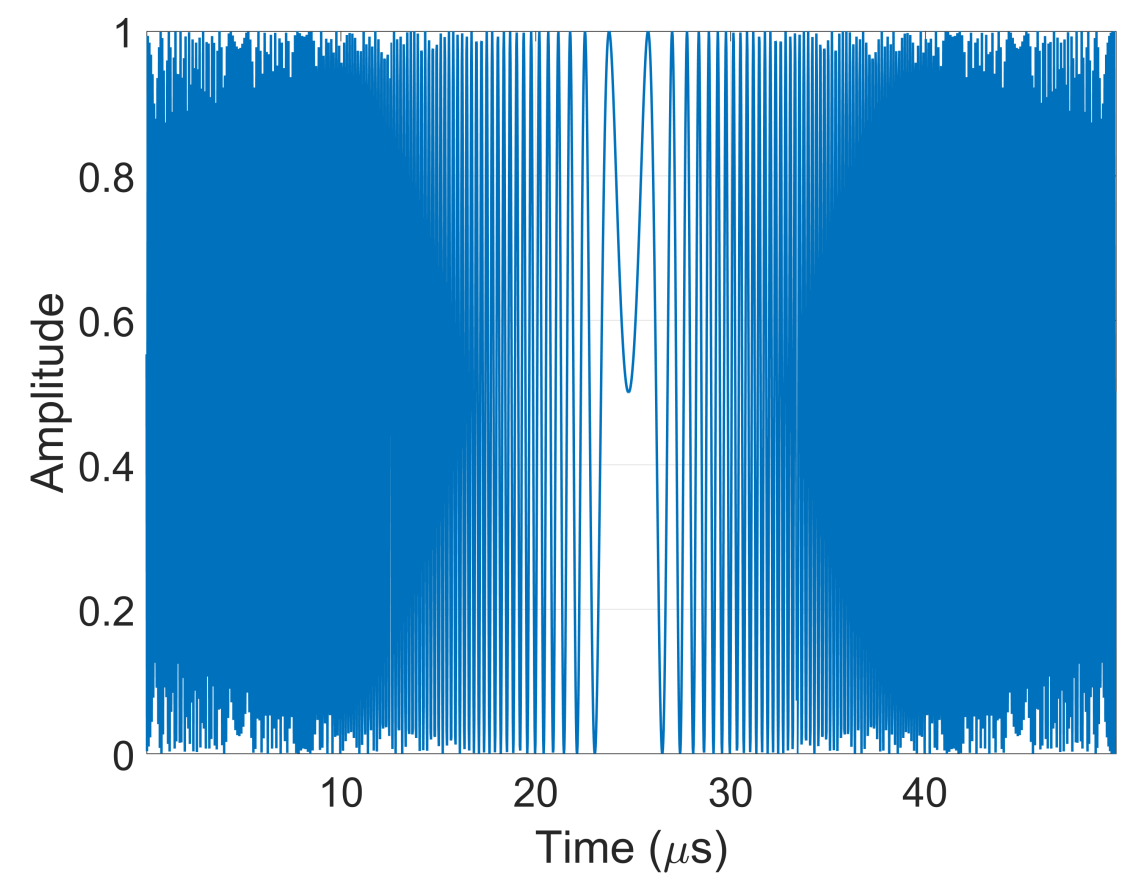

Figure 36: Convex NLFM transmitted pulse generated in MATLAB 


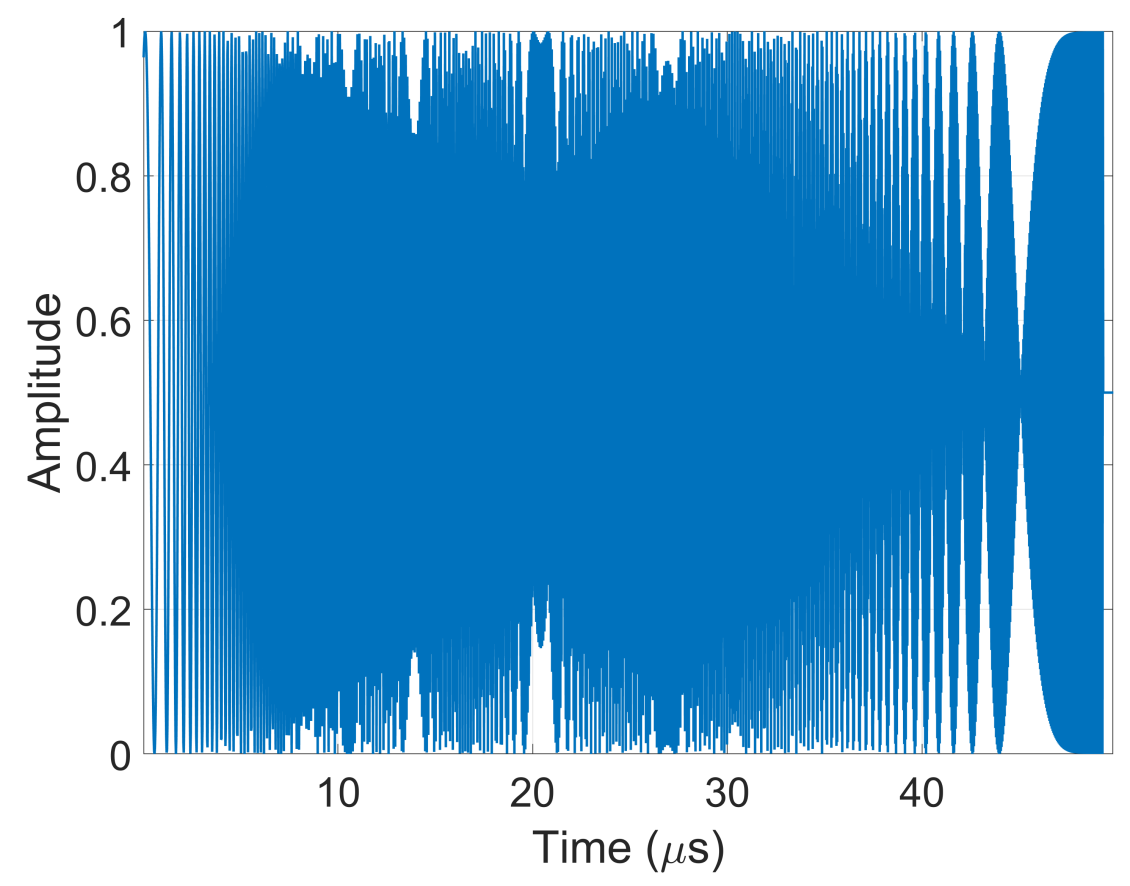

Figure 37: Concave NLFM transmitted pulse generated in MATLAB

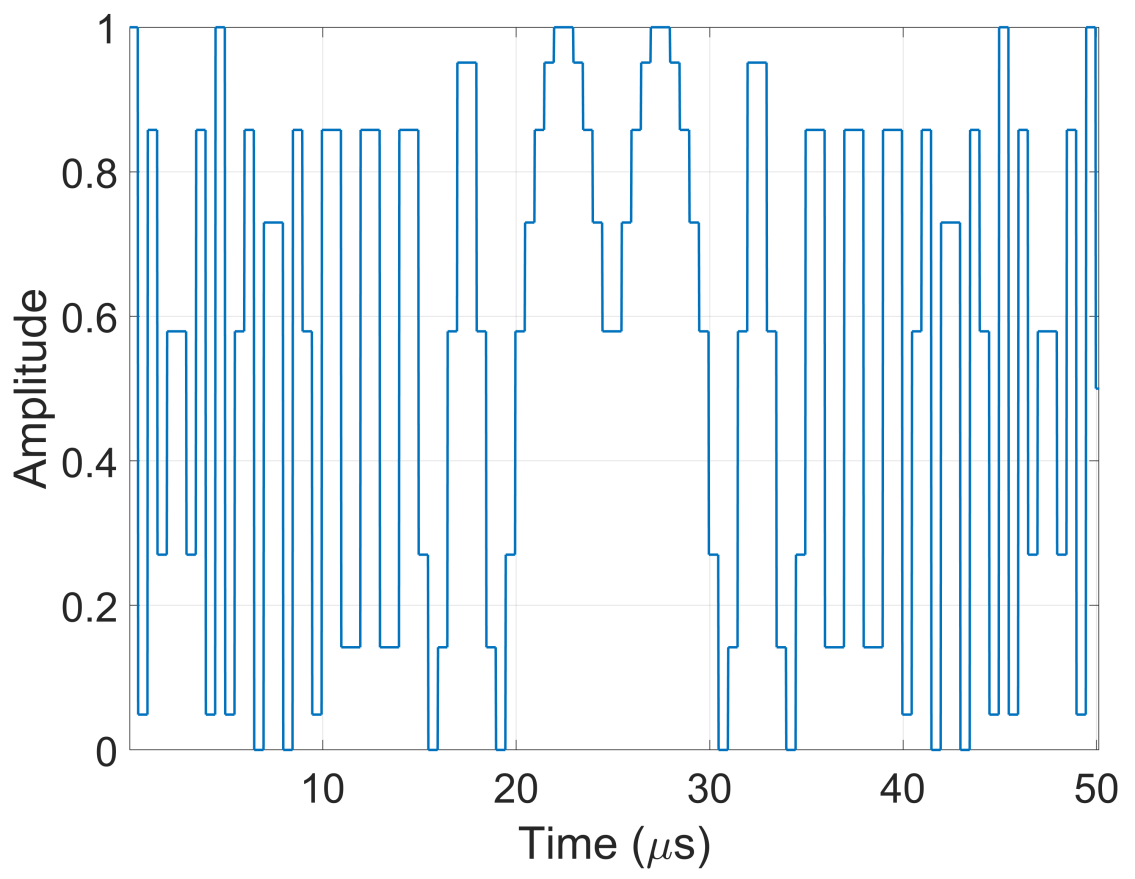

Figure 38: P2 transmitted pulse generated in MATLAB 


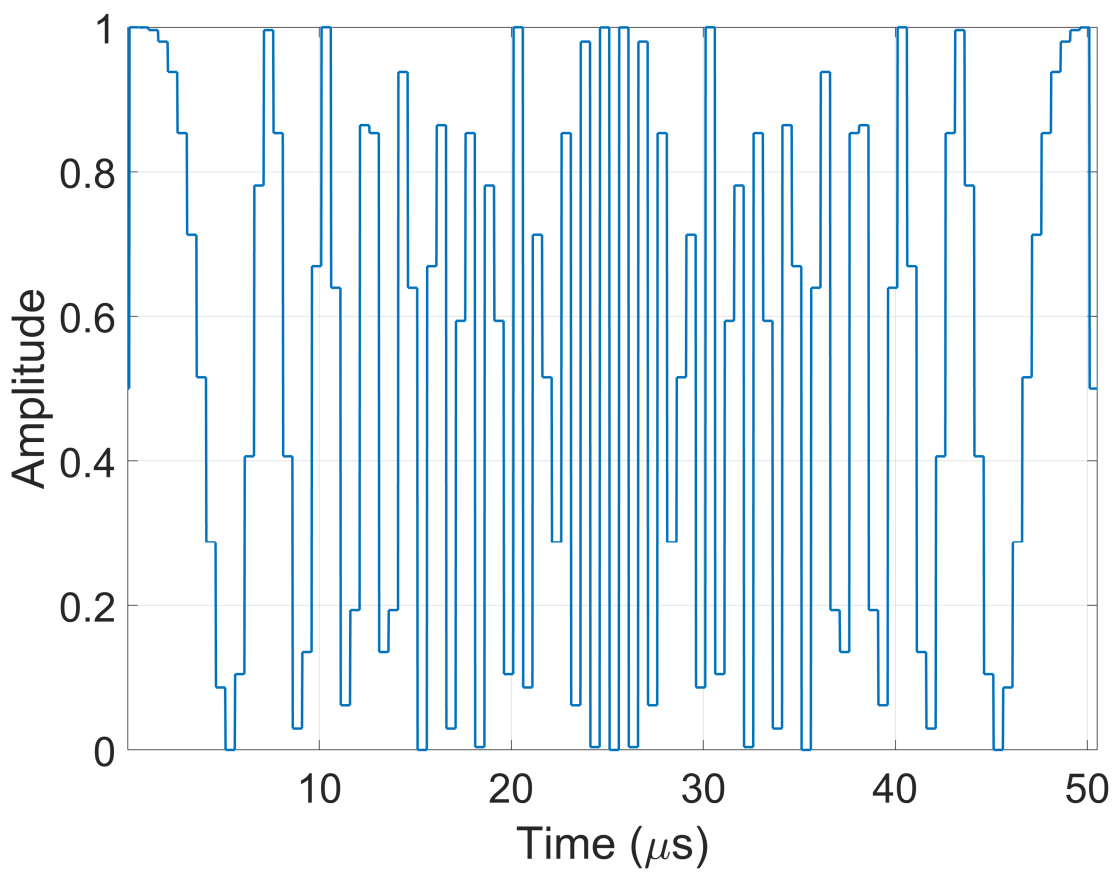

Figure 39: $\mathrm{P} 3$ transmitted pulse generated in MATLAB

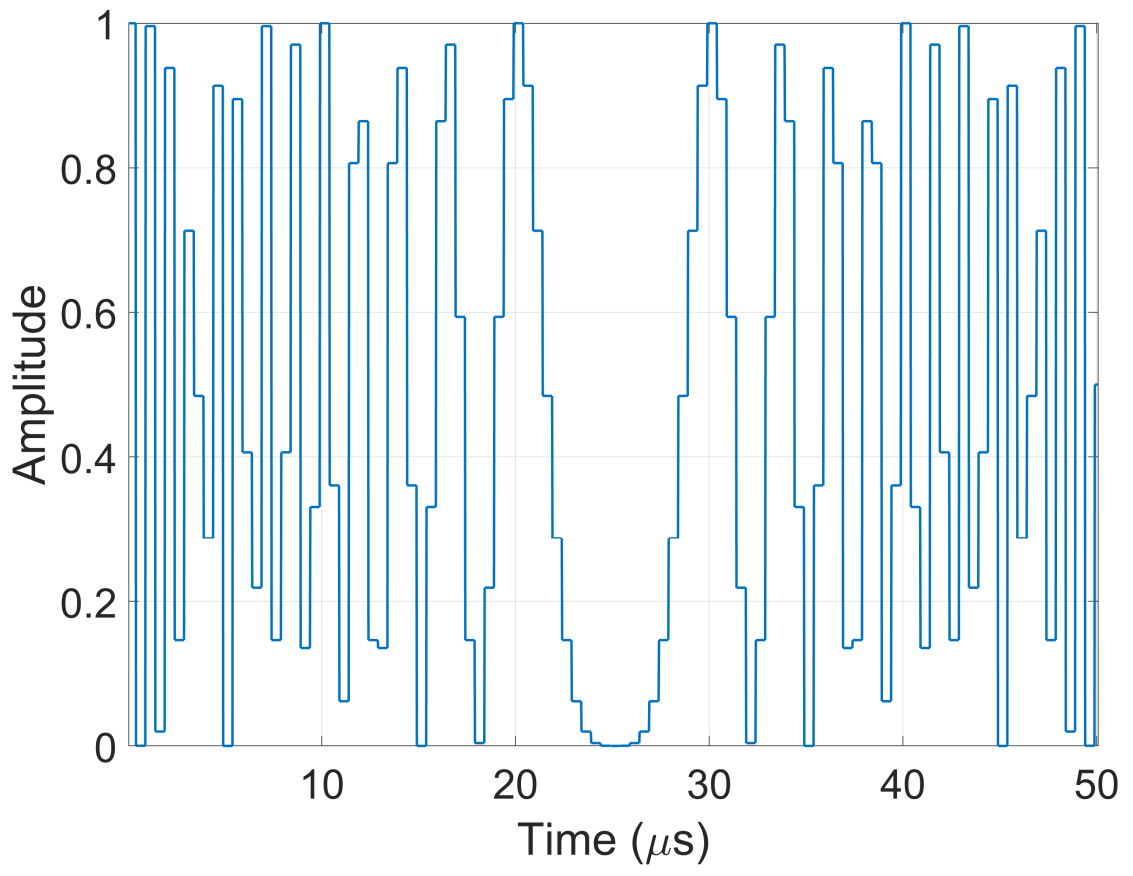

Figure 40: $\mathrm{P} 4$ transmitted pulse generated in MATLAB 


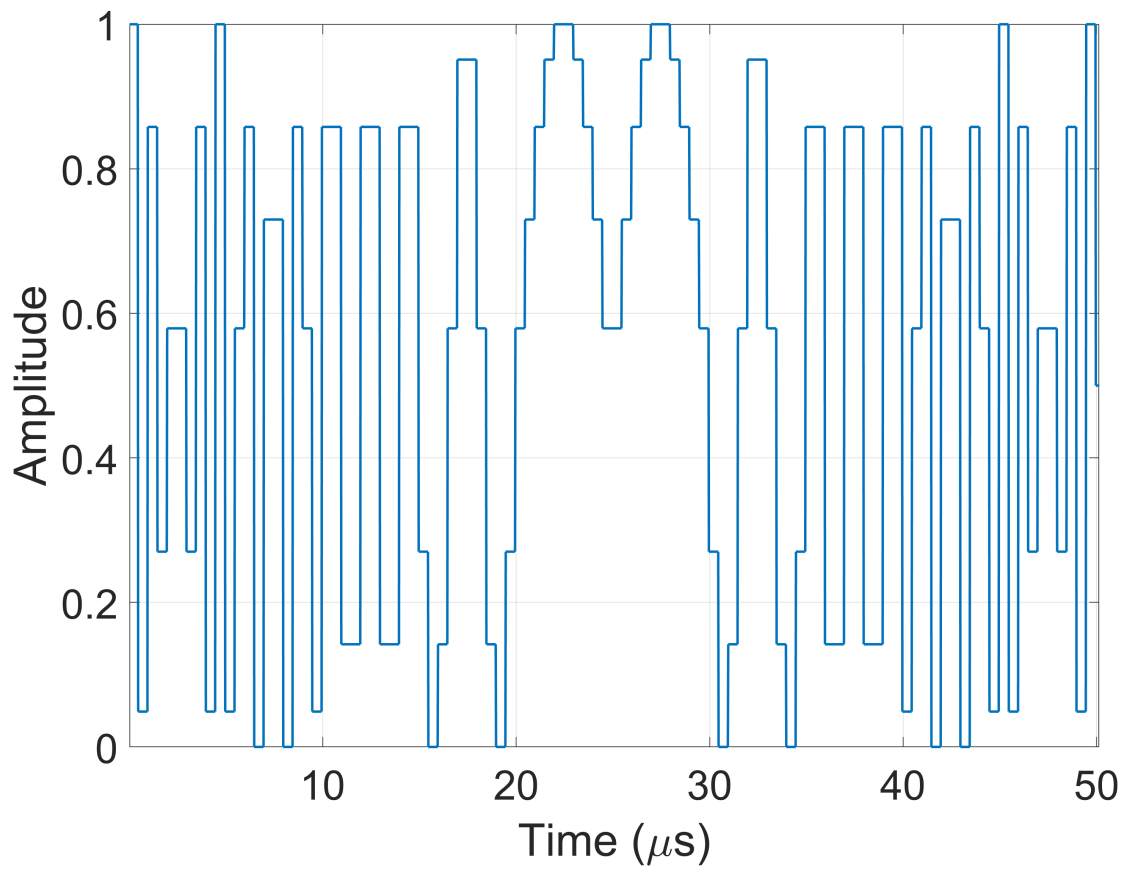

Figure 41: Px transmitted pulse generated in MATLAB 


\section{Appendix B}

\section{MATLAB Code}

Algorithm 3: signal class

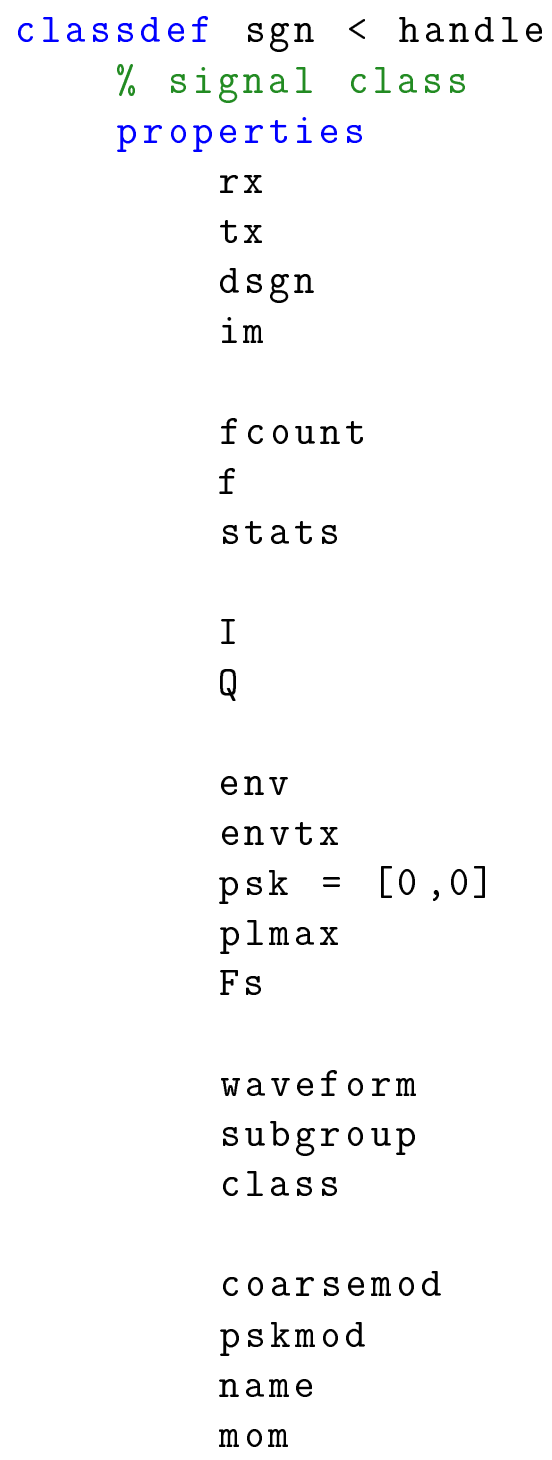




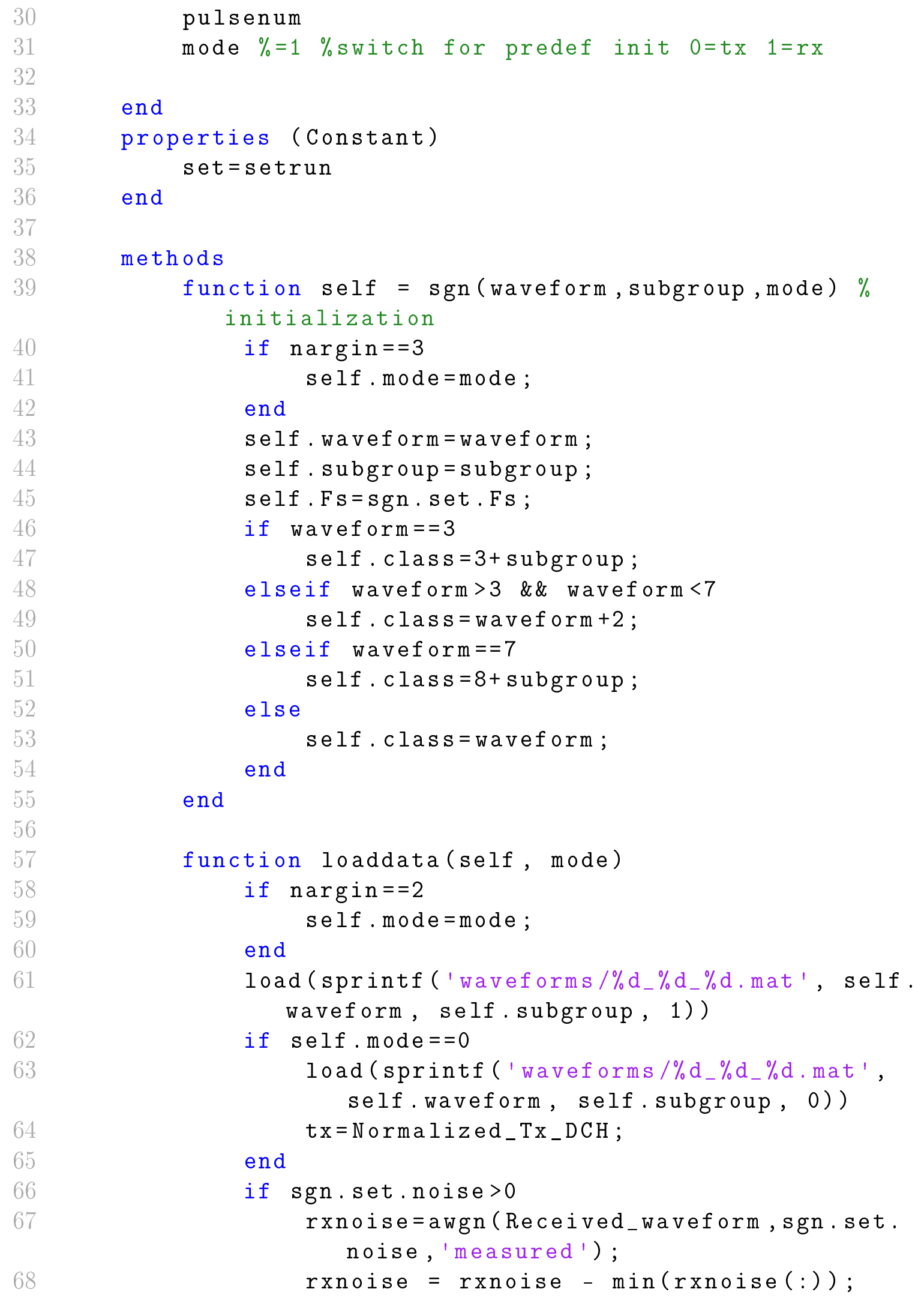




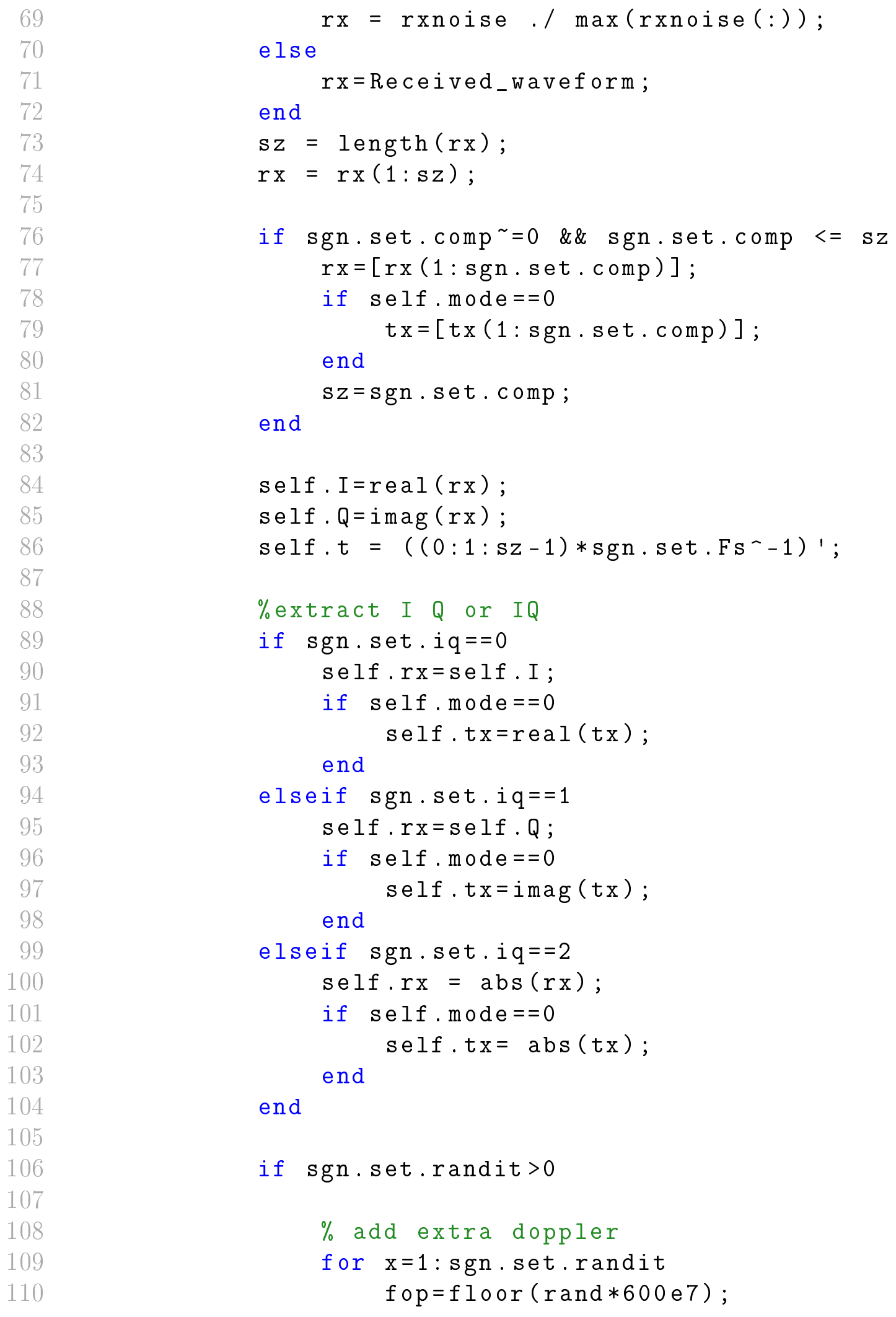




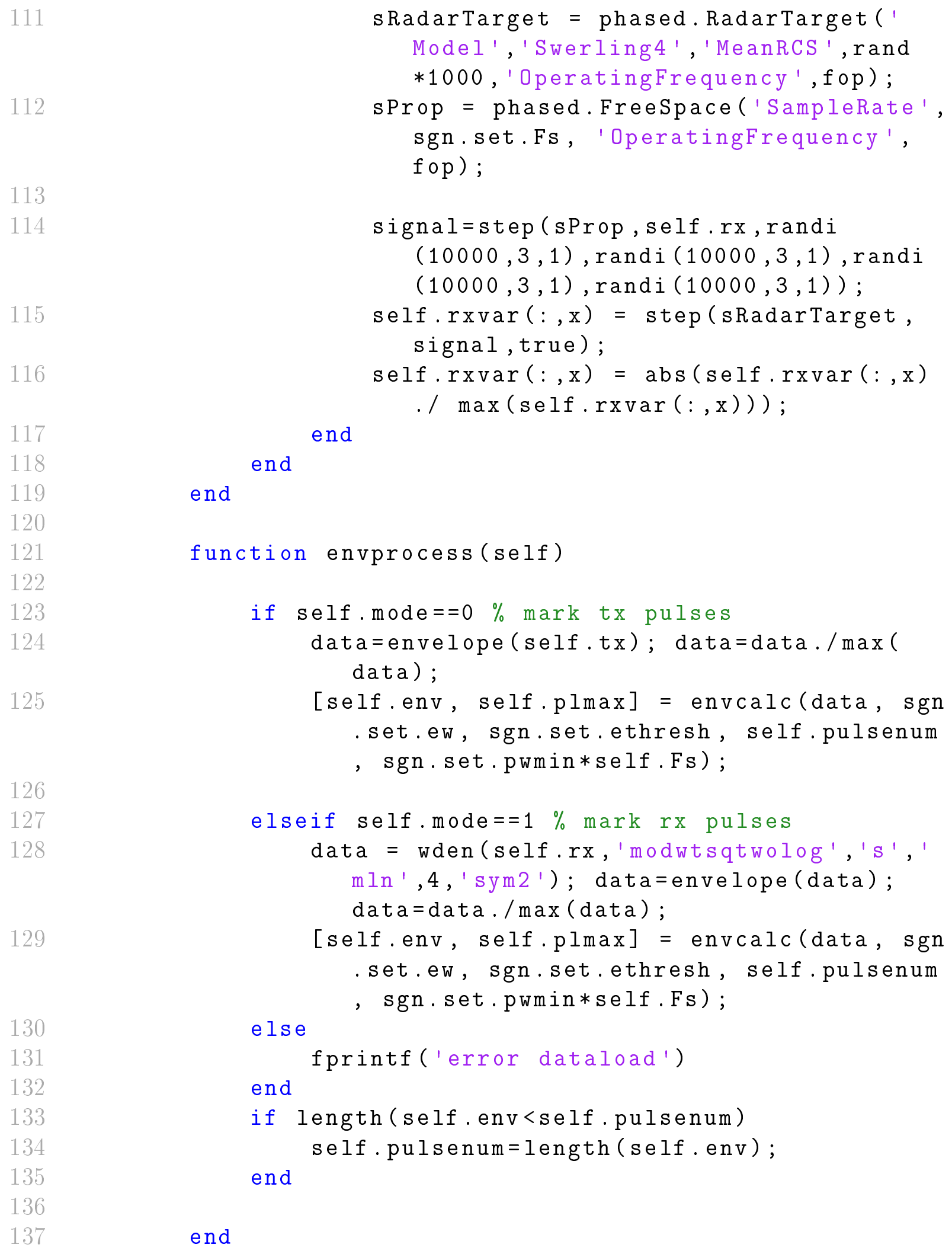


139

function HDTclassify(self)

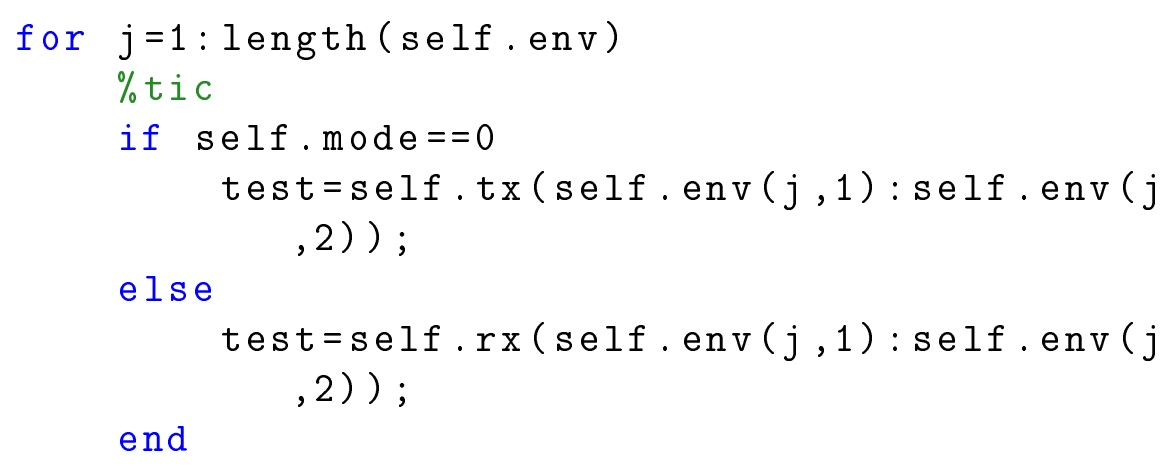

end

function ANNclassify(self, mom, par )

if self.psk $(1,2)==0$

self.psk=self.env;

disp('no PSK found')

end

self.env=self.psk;

tic

rclass $=$ self.class

$j j=1 ; r=1$;

if $\operatorname{par}==0$

for $j=1:$ length (self.env)

if self .mode $==0$

test=self.tx ( self.env $(j, 1)$ : self.env $(j$ ,2));

else

test $=\operatorname{self} \cdot r x(\operatorname{self} . \operatorname{env}(j, 1): \operatorname{self} \cdot \operatorname{env}(j$ ,2));

end

$[\operatorname{self} . \operatorname{dsgn}(j,:), \sim]=\operatorname{ANNfeatExt}$ (test, self. env, self.Fs, sgn.set.debugFM, mom, length (self.psk));

end

els e

if self. mode $==0$ test $=\operatorname{self} . t x$; 
175

176

177

178

179

180

181

182

183 end else test $=$ self.rx; end self.dsgn= ANNfeatExtPar(test, self.env, self.Fs, sgn.set.debugFM, mom, length( self.psk)) ;

end

fprintf ('extracted PSK in \%4.2f $\backslash n$ ', toc)

end end 
Algorithm 4: HDTfeatExt function

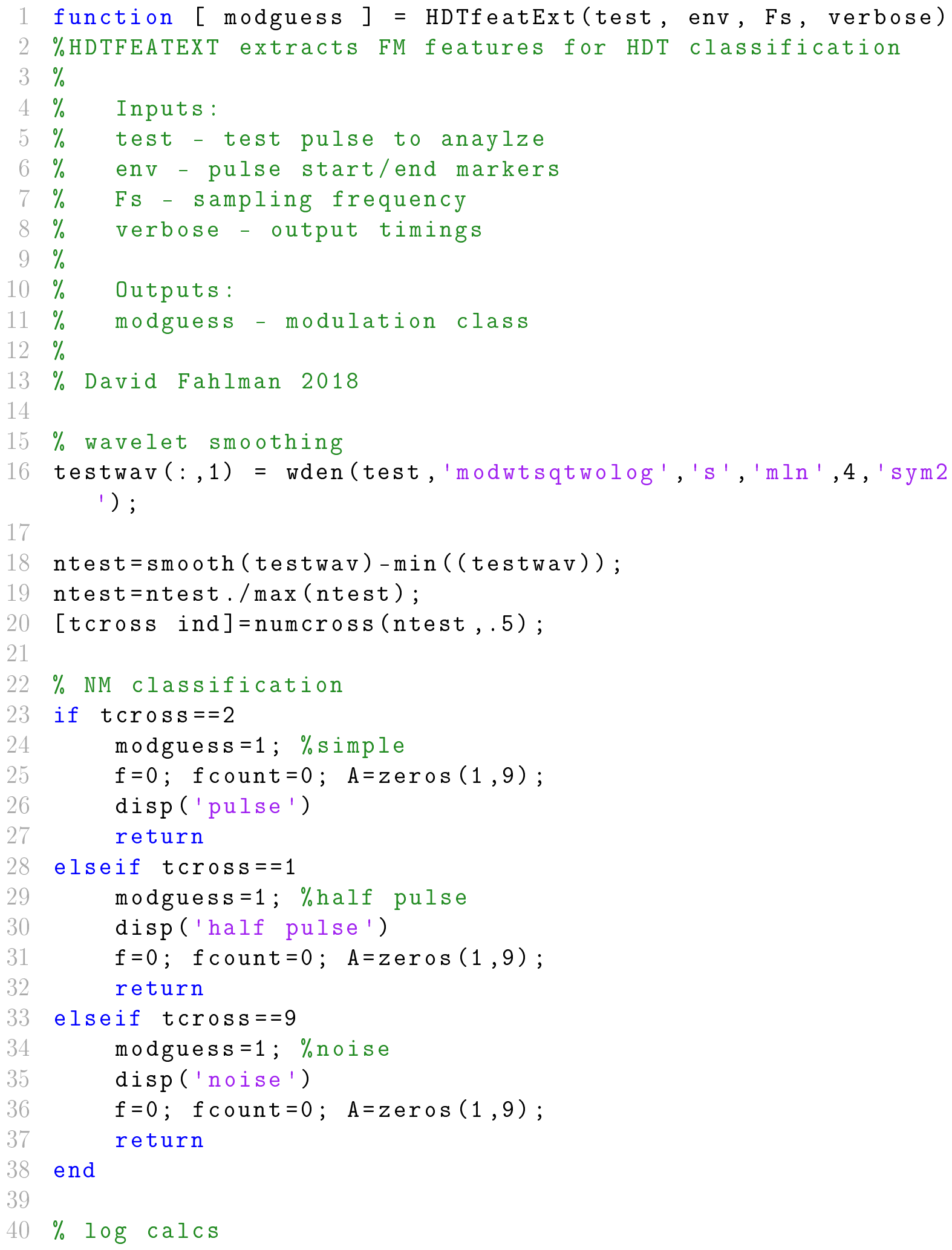




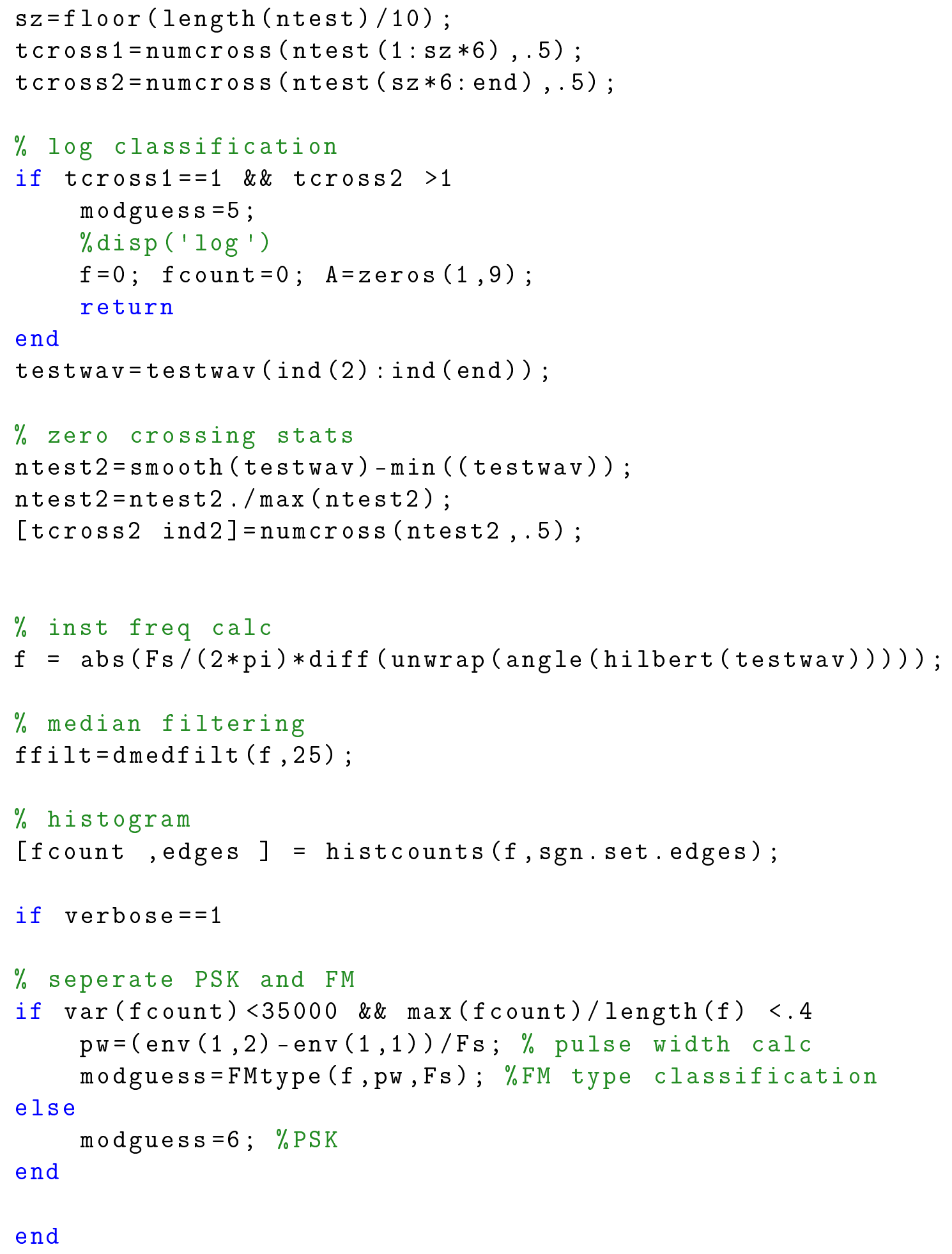


Algorithm 5: ANNfeatExt function

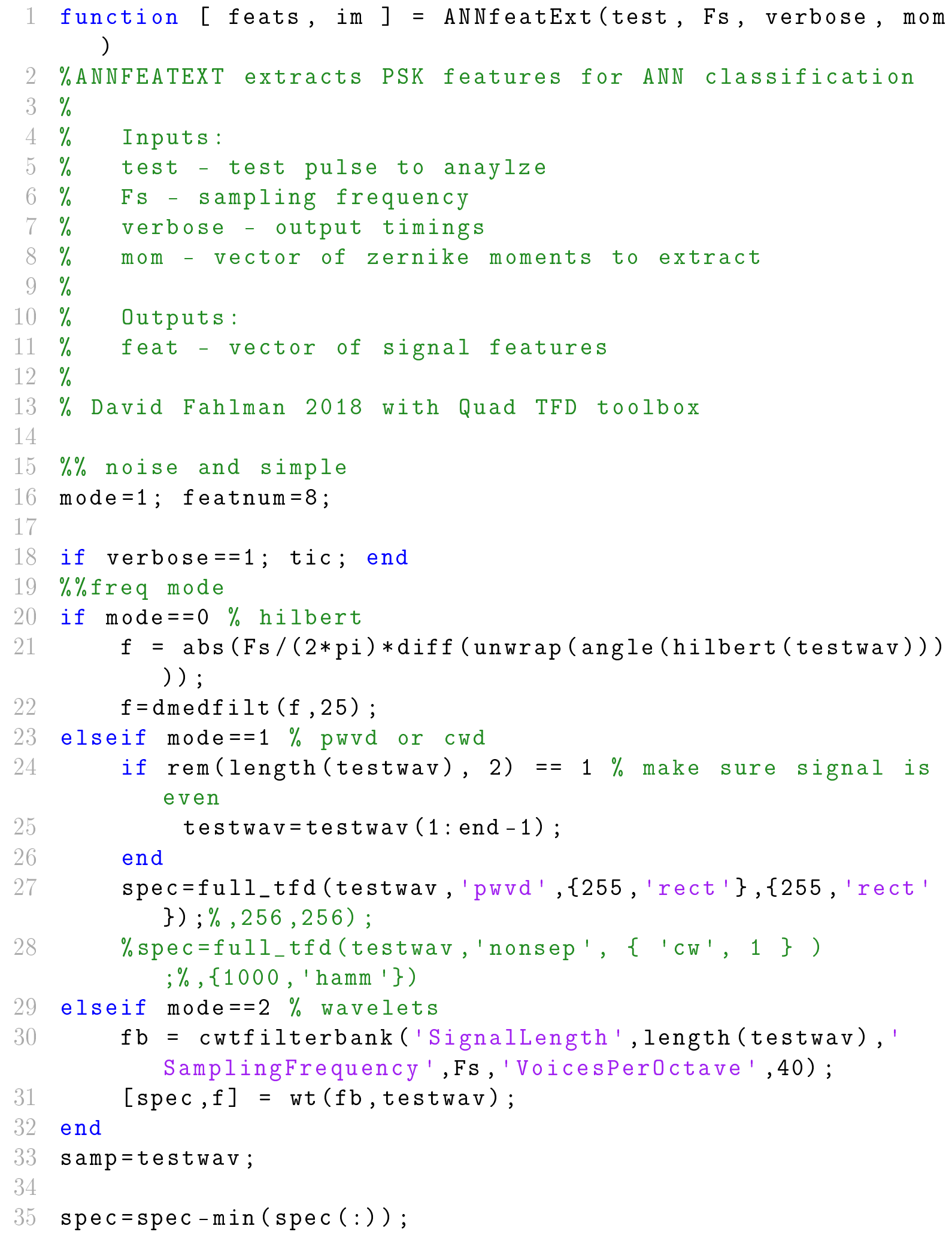


36 spec $=\operatorname{spec} . / \max (\operatorname{spec}(:)) ;$

$38 \mathrm{f}=\max (\operatorname{spec},[], 1) * \mathrm{Fs}$;

$39 f=\operatorname{dmedfilt}(\mathrm{f}, 12)$;

40

41 if verbose==1; fprintf ('Time to make spec $\% 4.2 f \backslash n^{\prime}$, toc); tic; end

42

43 im=normthresh (spec, samp); \%*100);

44

45 feats=featcalc (f, spec, samp);

47 if verbose==1; fprintf ('Time to make image \%4.2f \n',toc); tic; end

49 zern=calcZernike (im, mom); \%sz=length (zern) +5 ;

50 feats $=[$ feats zern];

51

52 if verbose==1; fprintf ('Time to make zernike \%4.2f \n', toc ); end

53

54 end 


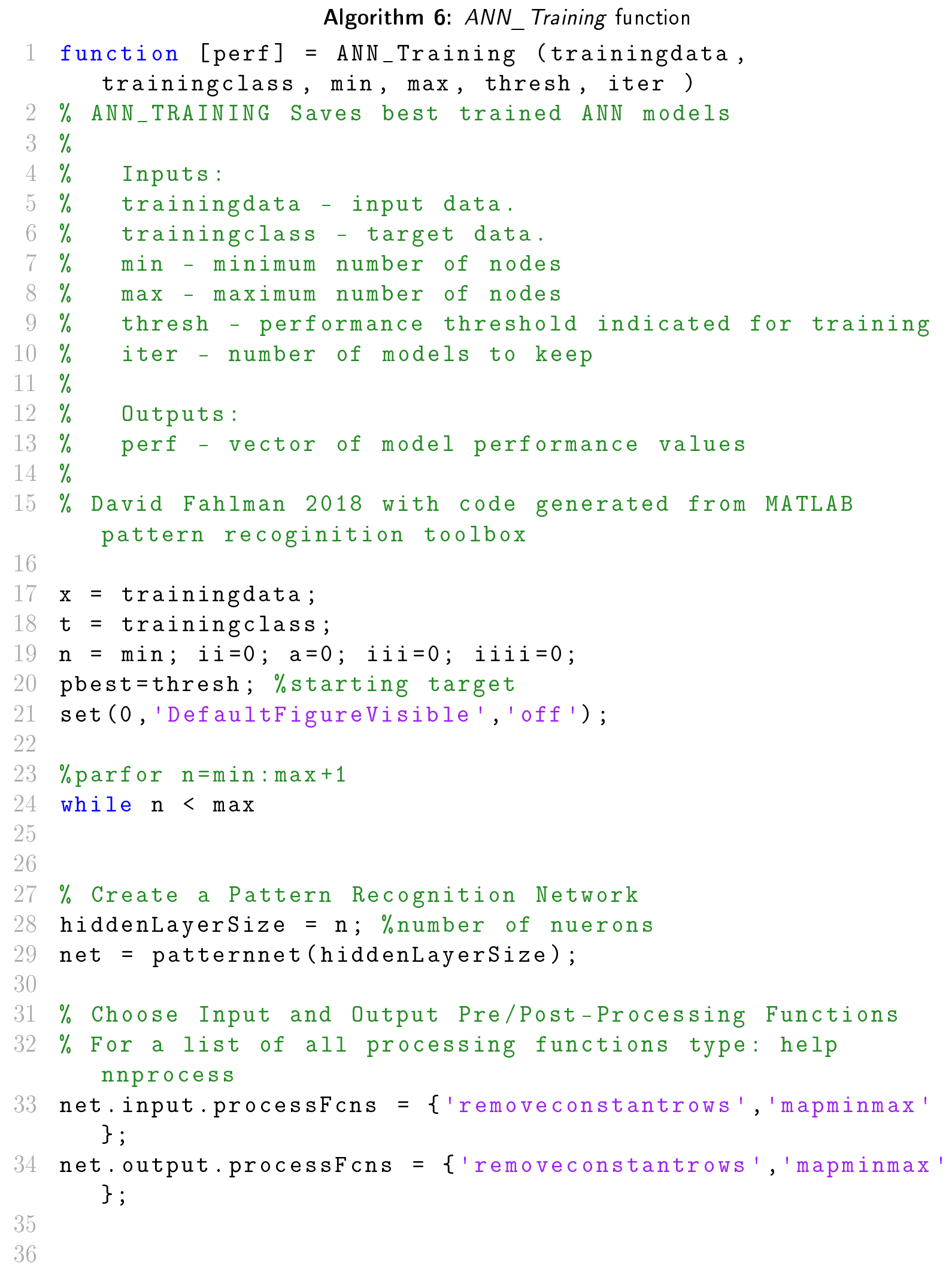

$27 \%$ Create a Pattern Recognition Network

28 hiddenLayerSize $=\mathrm{n} ; \%$ number of nuerons

29 net $=$ patternnet (hiddenLayerSize);

$31 \%$ Choose Input and Output Pre/Post-Processing Functions

$32 \%$ For a list of all processing functions type: help nnprocess

33 net.input.processfcns = \{'removeconstantrows', 'mapminmax' \}

34 net.output.processfcns $=$ \{'removeconstantrows ', 'mapminmax' \} 
$37 \%$ Setup Division of Data for Training, Validation, Testing

$38 \%$ For a list of all data division functions type: help nndivide

39 net.divideFcn = 'dividerand '; \% Divide data randomly

40 net.divideMode = 'sample'; \% Divide up every sample

41 net.divideParam.trainRatio $=70 / 100$;

42 net.divideParam.valRatio = $15 / 100$

43 net.divideParam.testRatio $=15 / 100$;

$45 \%$ For help on training function 'trainscg' type: help trainscg

$46 \%$ For a list of all training functions type: help nntrain

47 net.trainfcn = 'trainscg'; \% Scaled conjugate gradient

$49 \%$ Choose a Performance Function

$50 \%$ For a list of all performance functions type: help nnperformance

51 net.performFcn = 'crossentropy'; \% Cross-entropy

$53 \%$ Choose Plot Functions

$54 \%$ For a list of all plot functions type: help nnplot

55 net.plotFcns $=$ \{'plotperform', 'plottrainstate' ', ploterrhist', ...

56 'plotregression', 'plotfit'\};

58 net.trainParam. showwindow $=0$;

$59 \%$ Train the Network

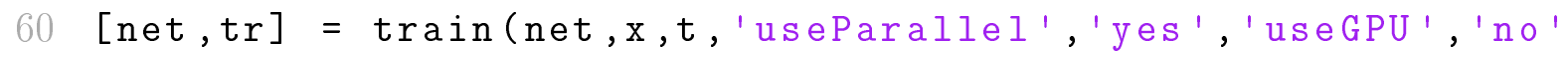
, 'showResources ', ' no' );

$62 \%$ Test the Network

$63 \mathrm{y}=\operatorname{net}(\mathrm{x}$, 'useParallel', 'yes ', 'useGPU ', 'no' ', 'showResources ', 'no');

64 e $=$ gsubtract $(t, y)$;

65 tind $=$ vec2ind $(t)$;

$66 \mathrm{yind}=\operatorname{vec} 2$ ind $(\mathrm{y})$;

67 percentErrors = sum(tind $\sim$ yind)/numel (tind);

68 performance $=$ perform $($ net, $t, y)$;

$70 \%$ Recalculate Training, Validation and Test Performance

71 trainTargets $=\mathrm{t} . *$ tr.trainMask $\{1\}$;

72 valTargets $=\mathrm{t} . *$ tr.valMask $\{1\} ;$

73 testTargets $=t . *$ tr.testMask $\{1\}$; 


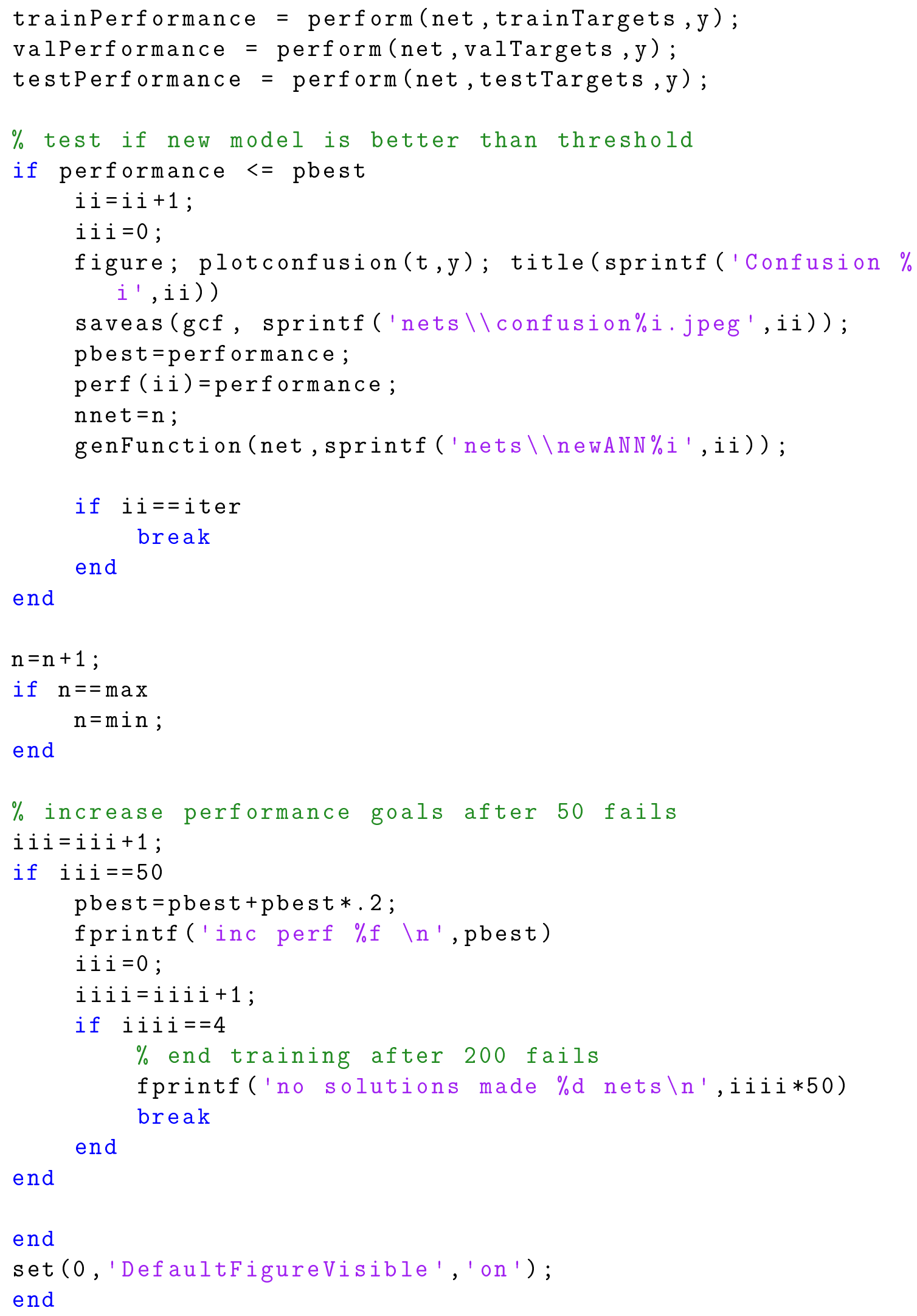


Appendix C

Additional Results

C.1 ANN Test 1 Confusion Matrices 


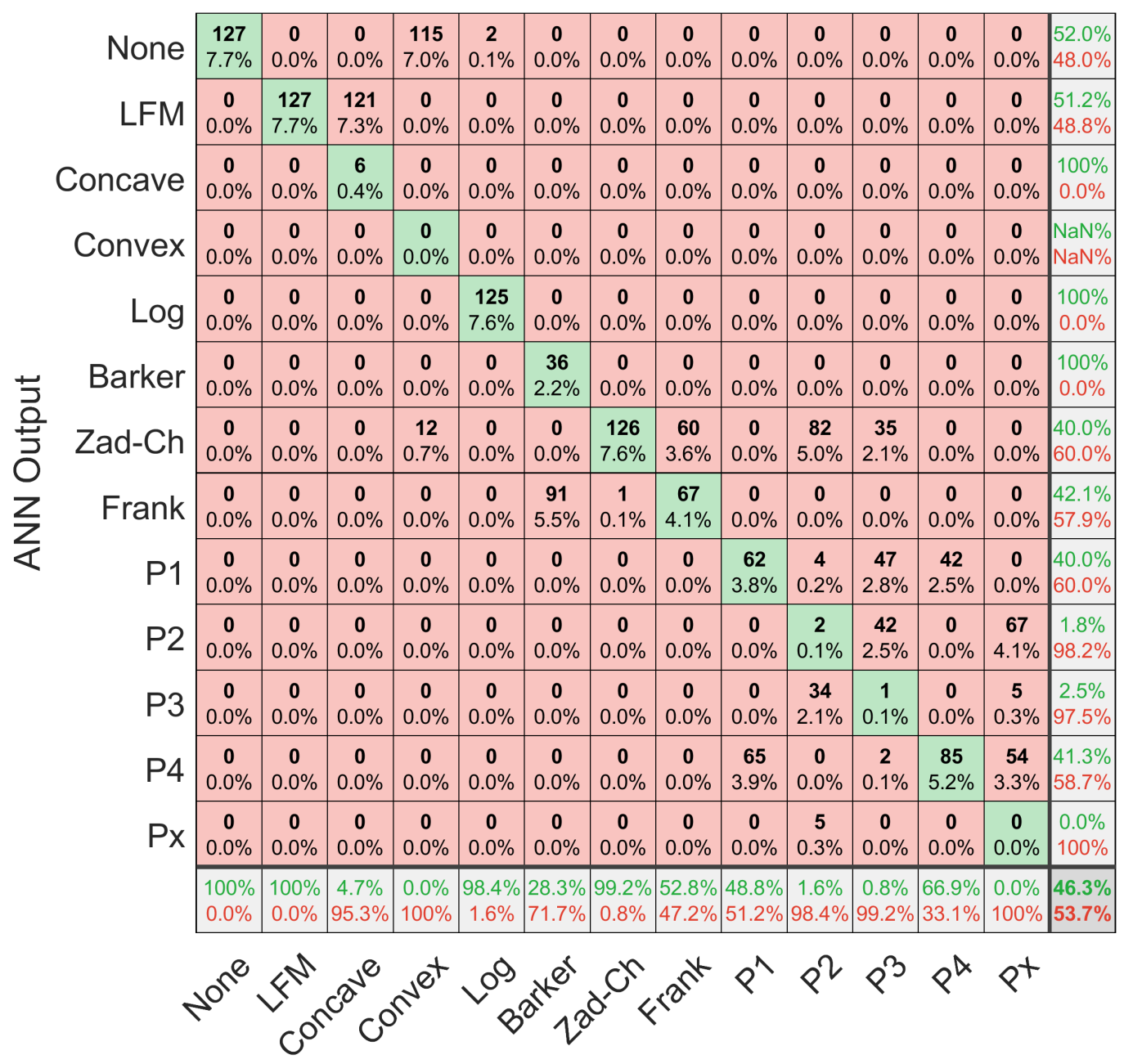

\section{True Class}

Figure 42: ANN Test 1 Confusion Matrix for $6 \mathrm{~dB}$ 


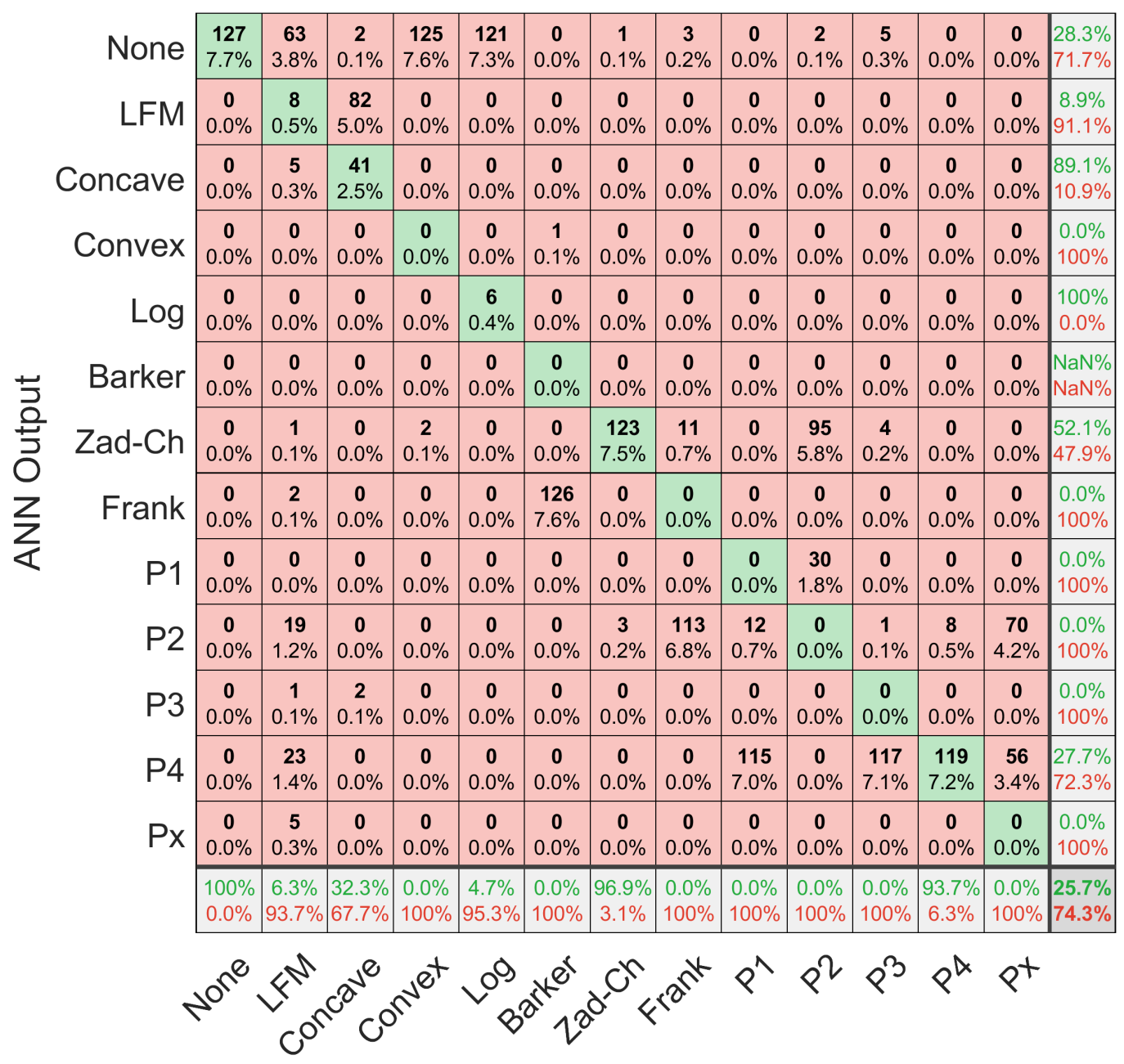

\section{True Class}

Figure 43: ANN Test 1 Confusion Matrix for $0 \mathrm{~dB}$ 


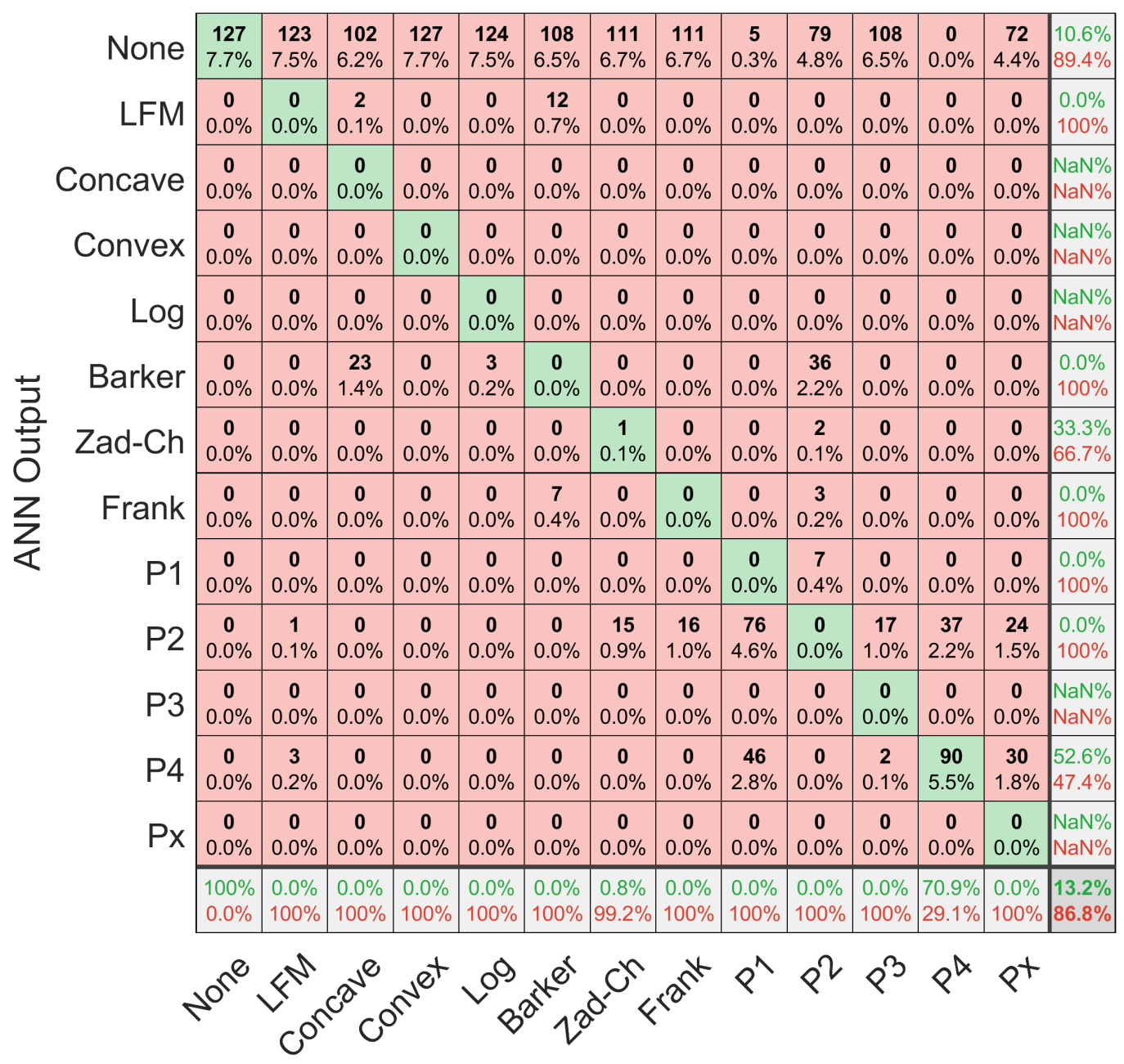

\section{True Class}

Figure 44: ANN Test 1 Confusion Matrix for $-6 \mathrm{~dB}$ 


\section{C.2 ANN Test 2 Confusion Matrices}

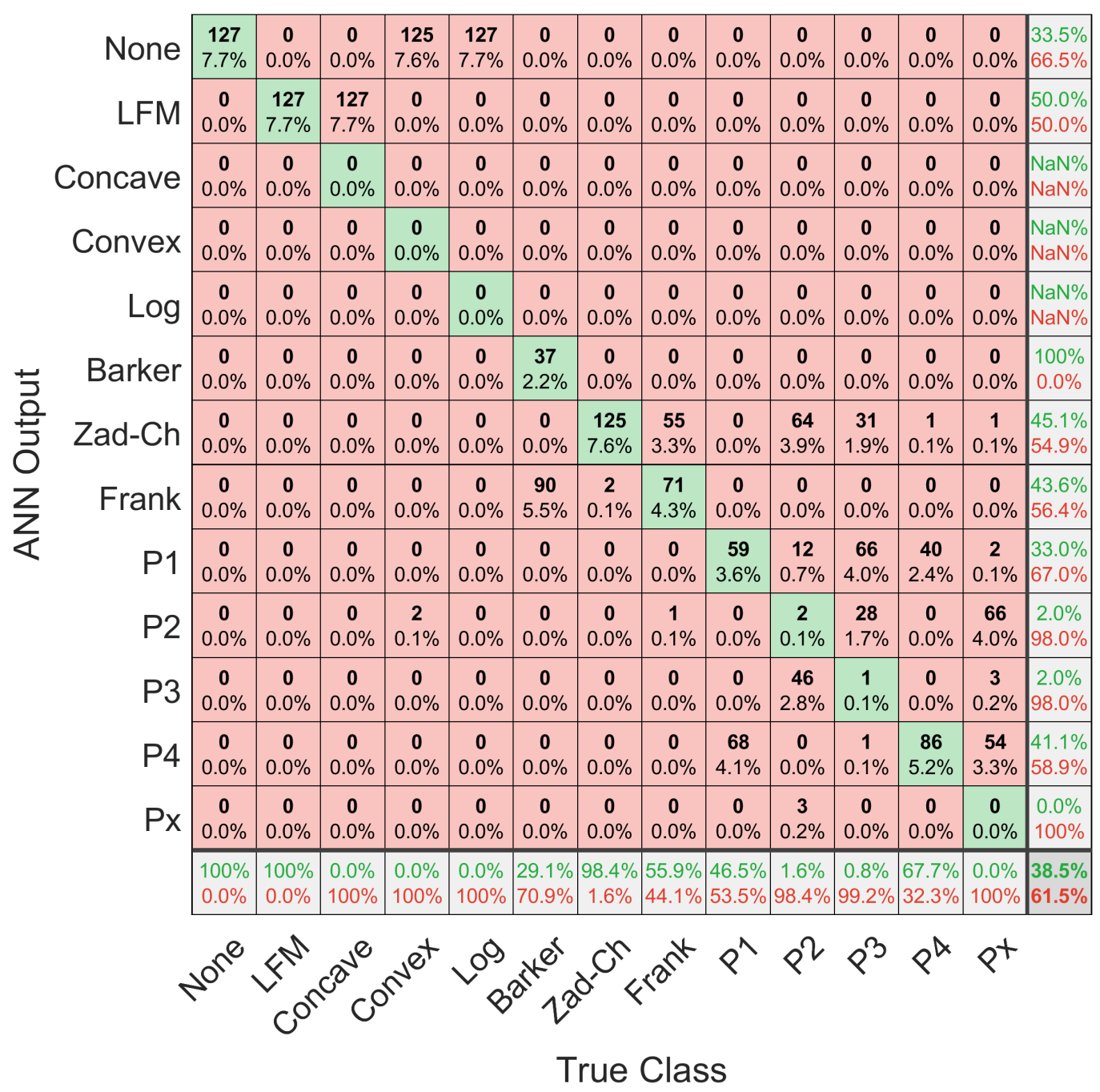

Figure 45: ANN Test 2 Confusion Matrix for $6 \mathrm{~dB}$ 


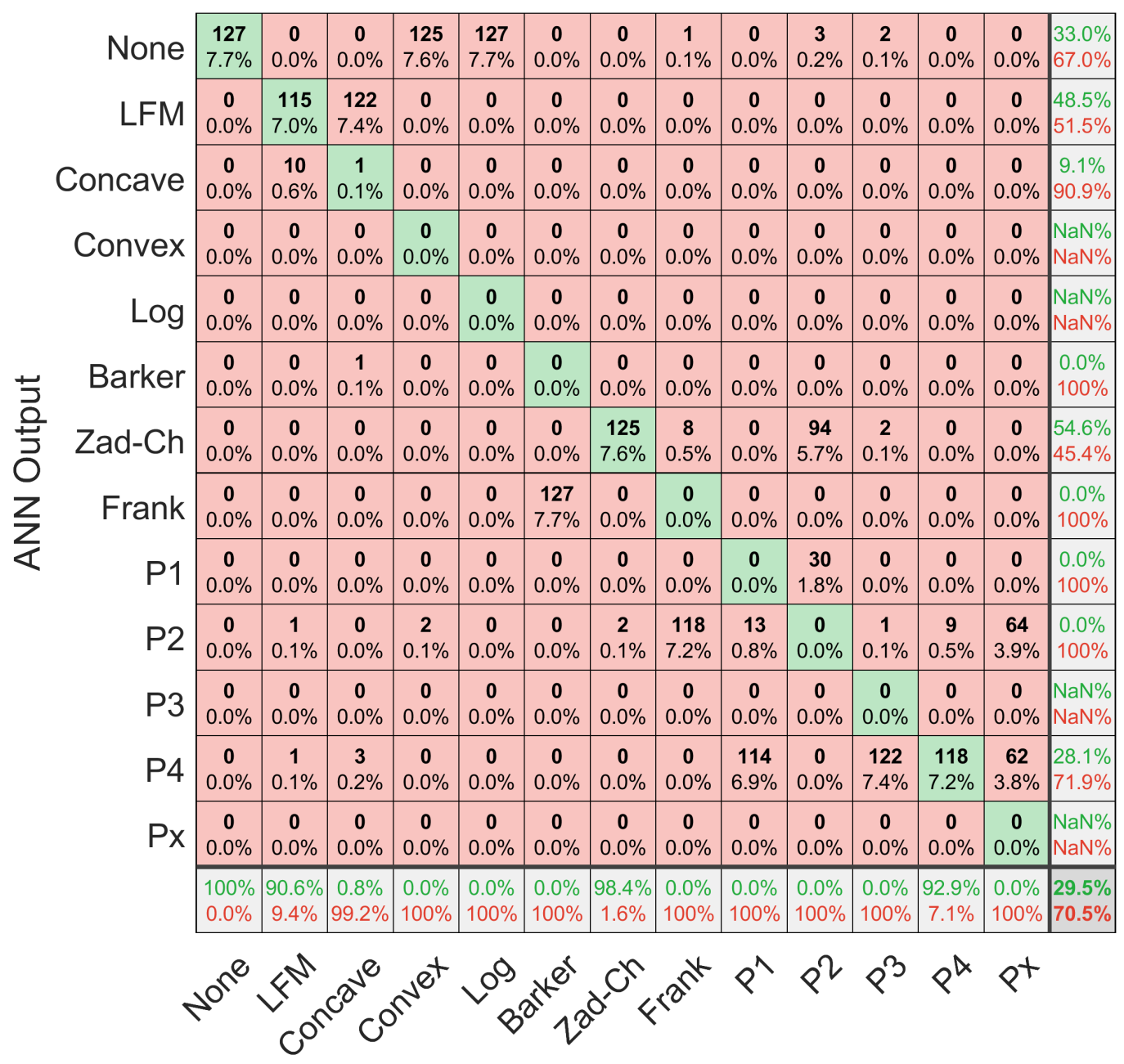

\section{True Class}

Figure 46: ANN Test 2 Confusion Matrix for $0 \mathrm{~dB}$ 


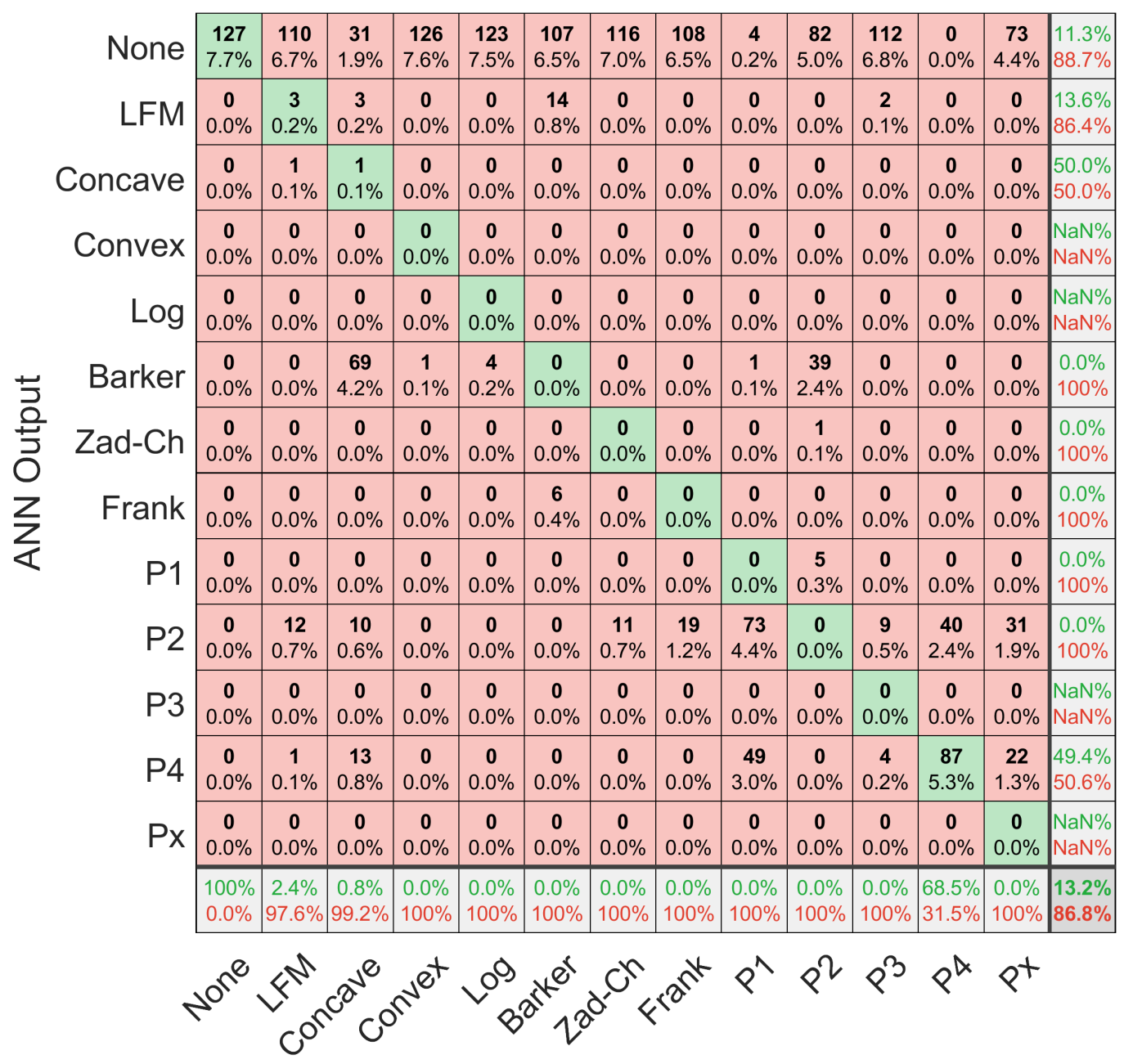

\section{True Class}

Figure 47: ANN Test 2 Confusion Matrix for $-6 \mathrm{~dB}$ 


\section{C.3 ANN Test 3 Confusion Matrices}

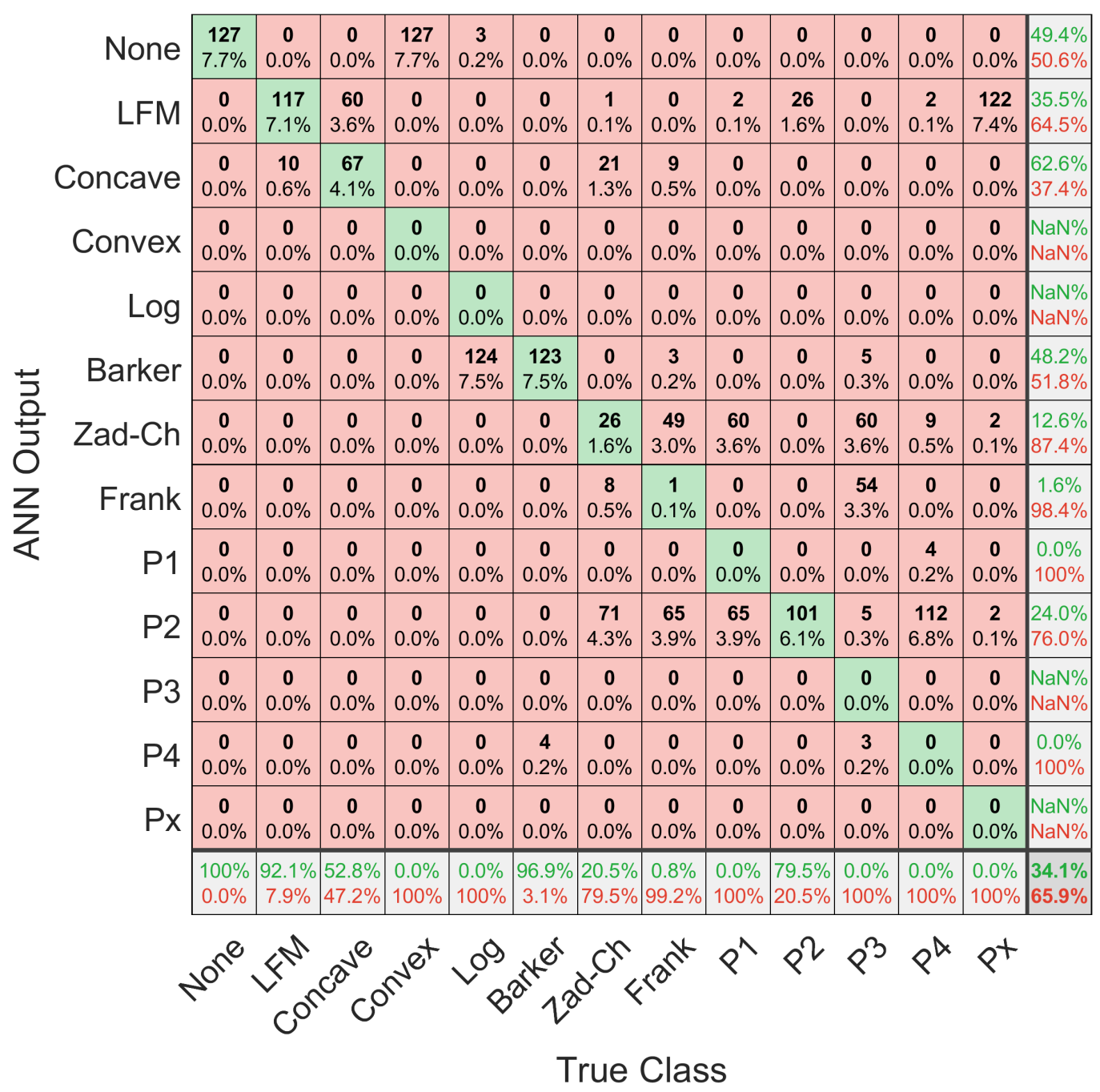

Figure 48: ANN Test 3 Confusion Matrix for $6 \mathrm{~dB}$ 


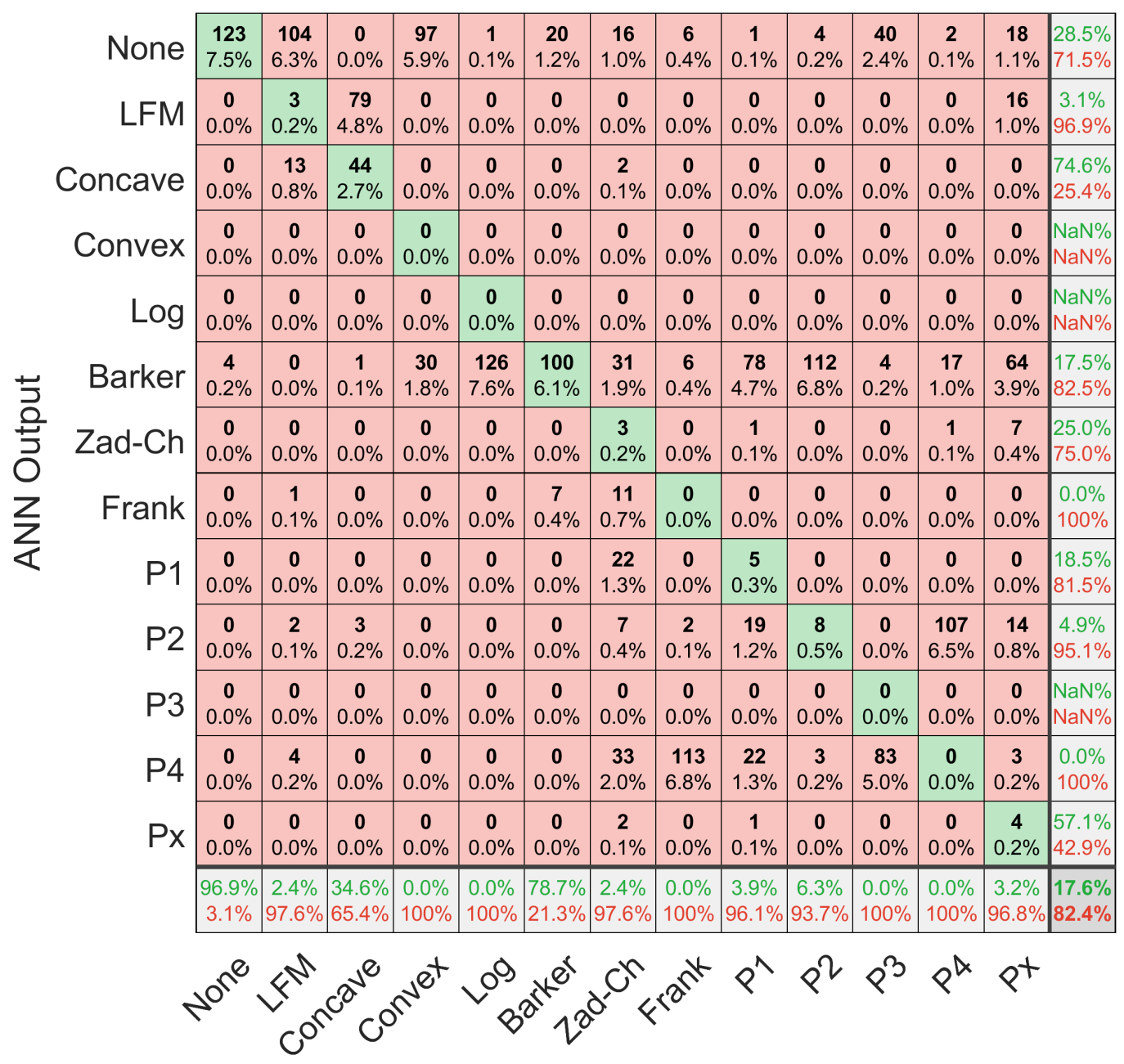

\section{True Class}

Figure 49: ANN Test 3 Confusion Matrix for $0 \mathrm{~dB}$ 


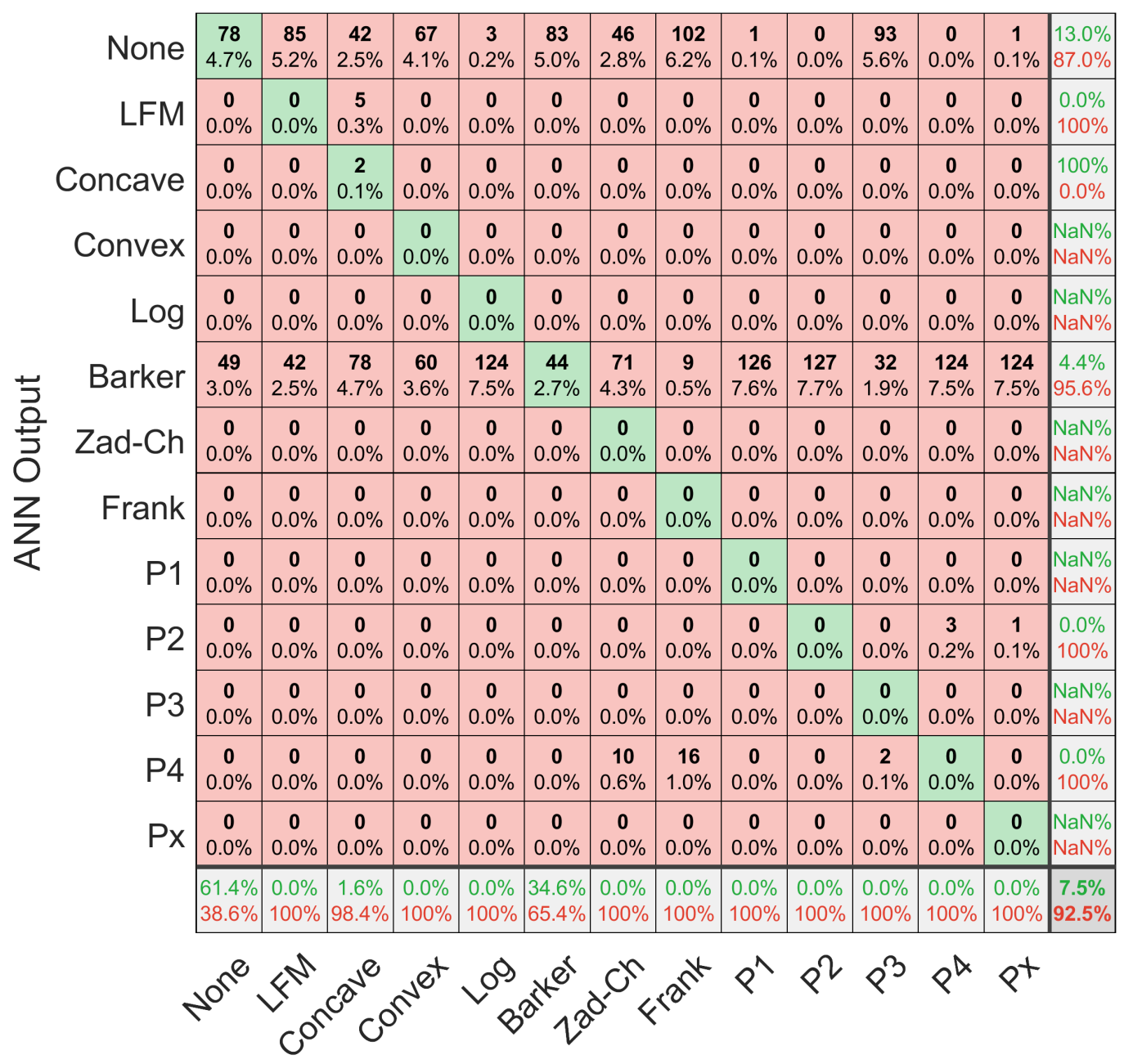

\section{True Class}

Figure 50: ANN Test 3 Confusion Matrix for $-6 \mathrm{~dB}$ 


\section{C.4 CNN Test 1 Confusion Matrices}

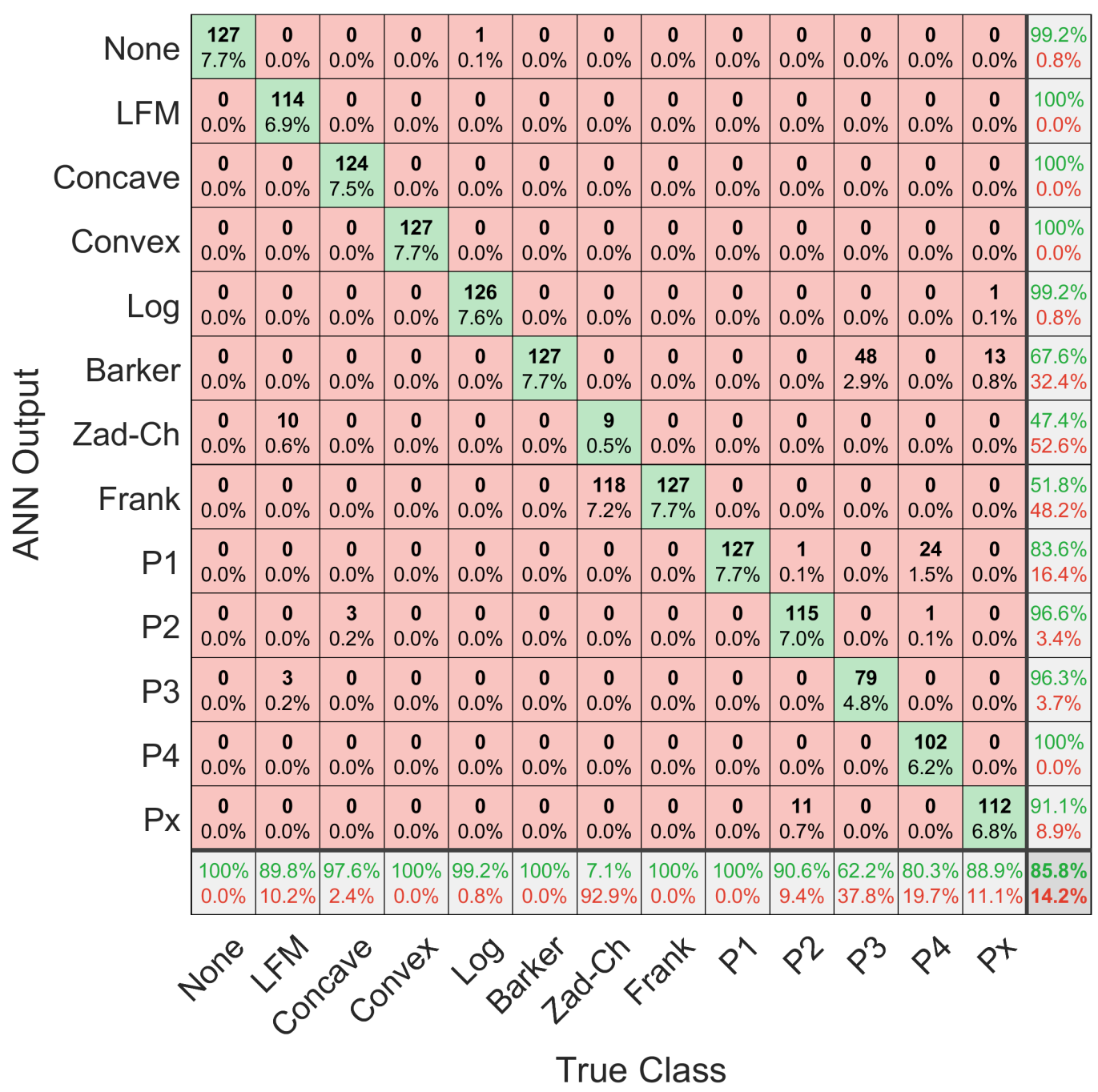

Figure 51: CNN Test 1 Confusion Matrix for $6 \mathrm{~dB}$ 


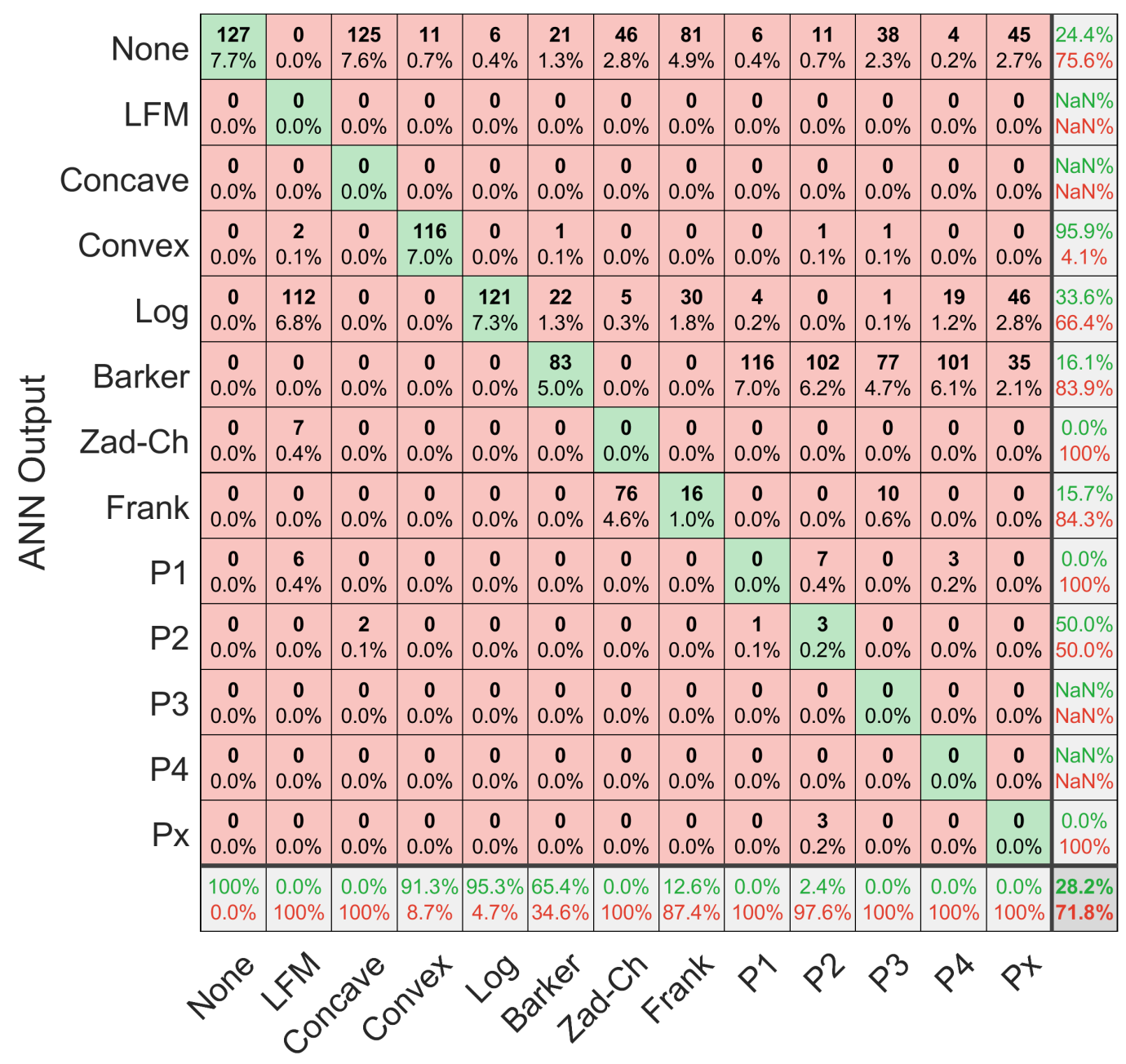

\section{True Class}

Figure 52: CNN Test 1 Confusion Matrix for $0 \mathrm{~dB}$ 


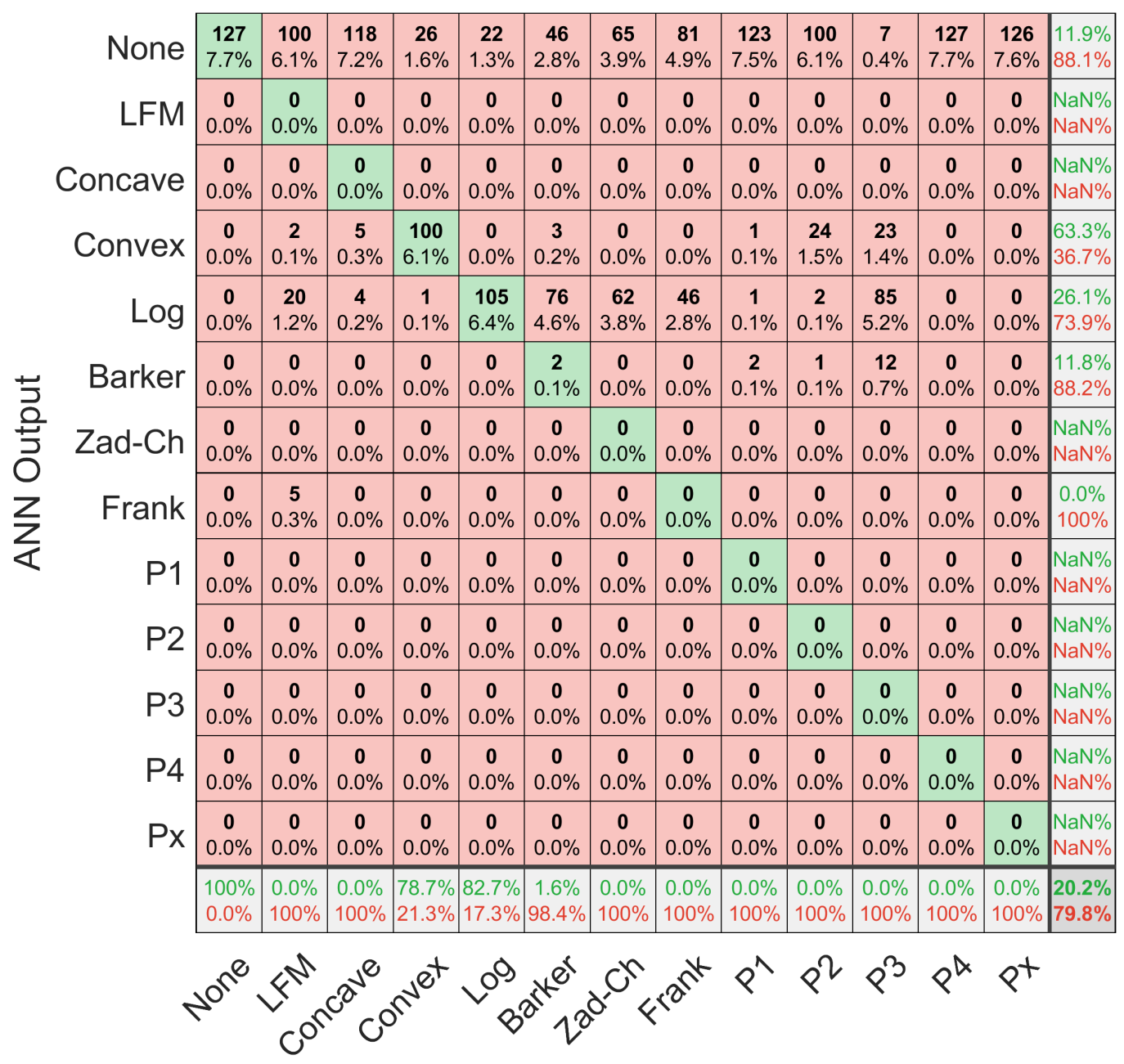

\section{True Class}

Figure 53: CNN Test 1 Confusion Matrix for $-6 \mathrm{~dB}$ 


\section{C.5 CNN Test 2 Confusion Matrices}

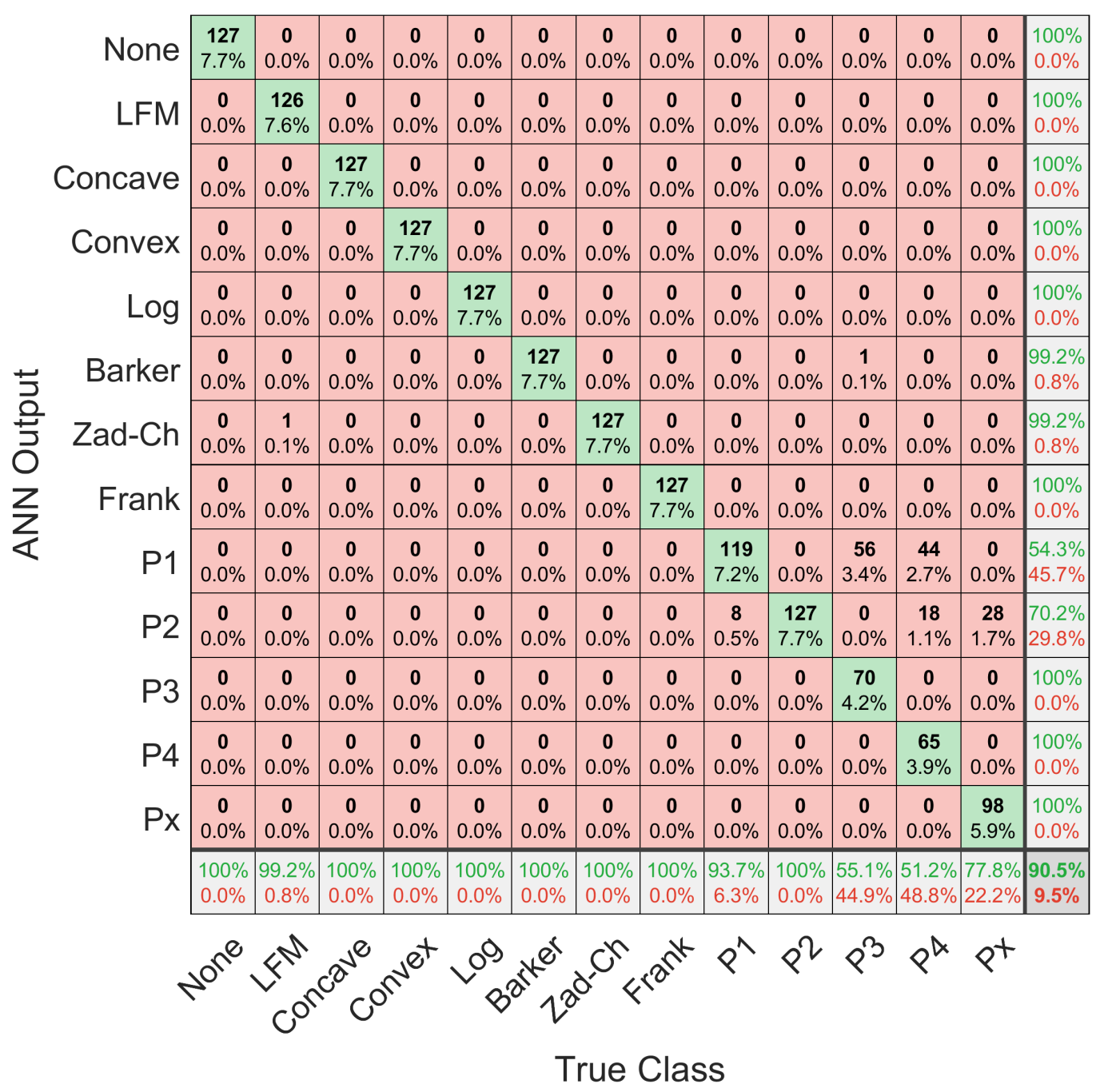

Figure 54: CNN Test 2 Confusion Matrix for $6 \mathrm{~dB}$ 


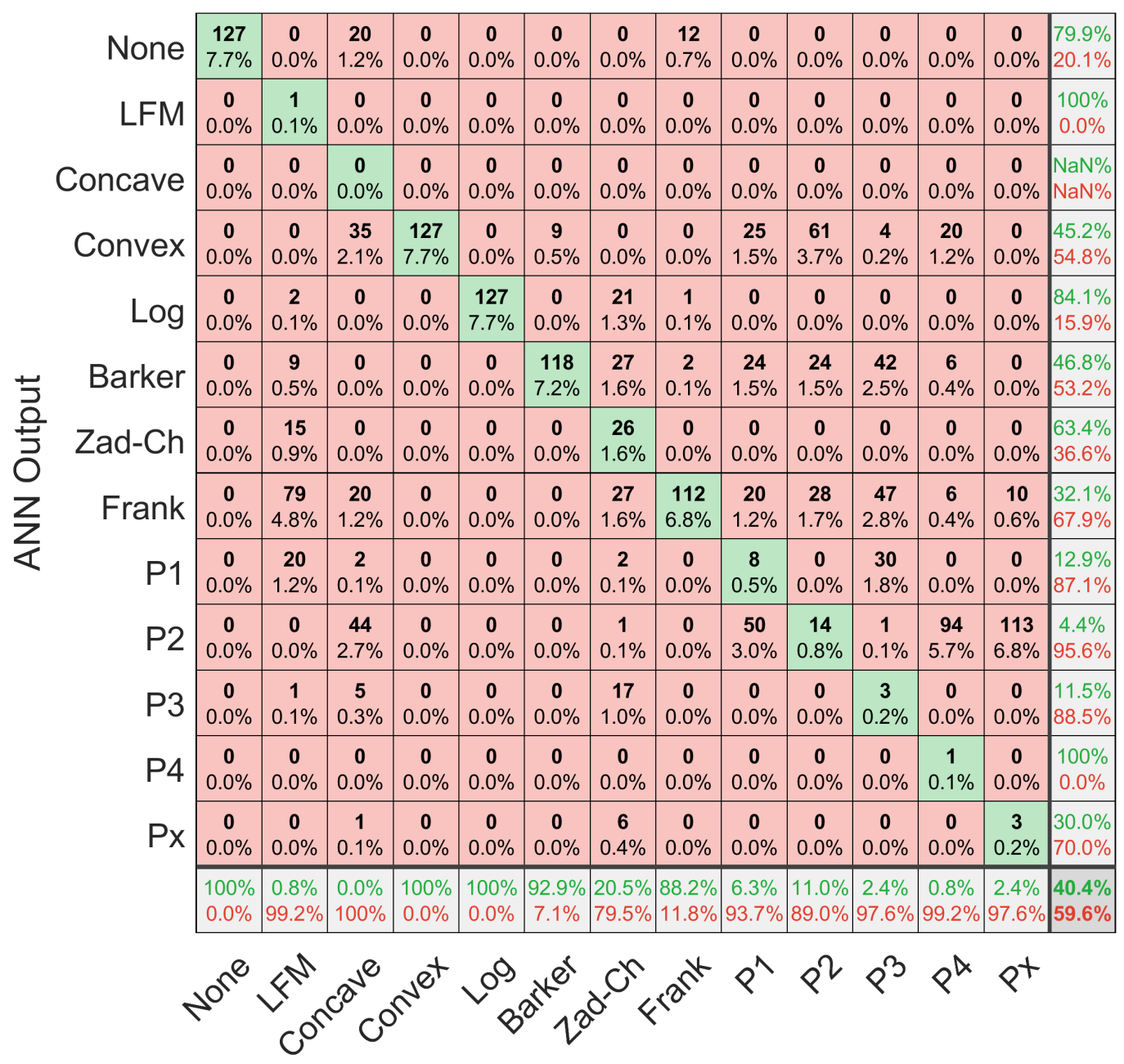

\section{True Class}

Figure 55: CNN Test 2 Confusion Matrix for $0 \mathrm{~dB}$ 


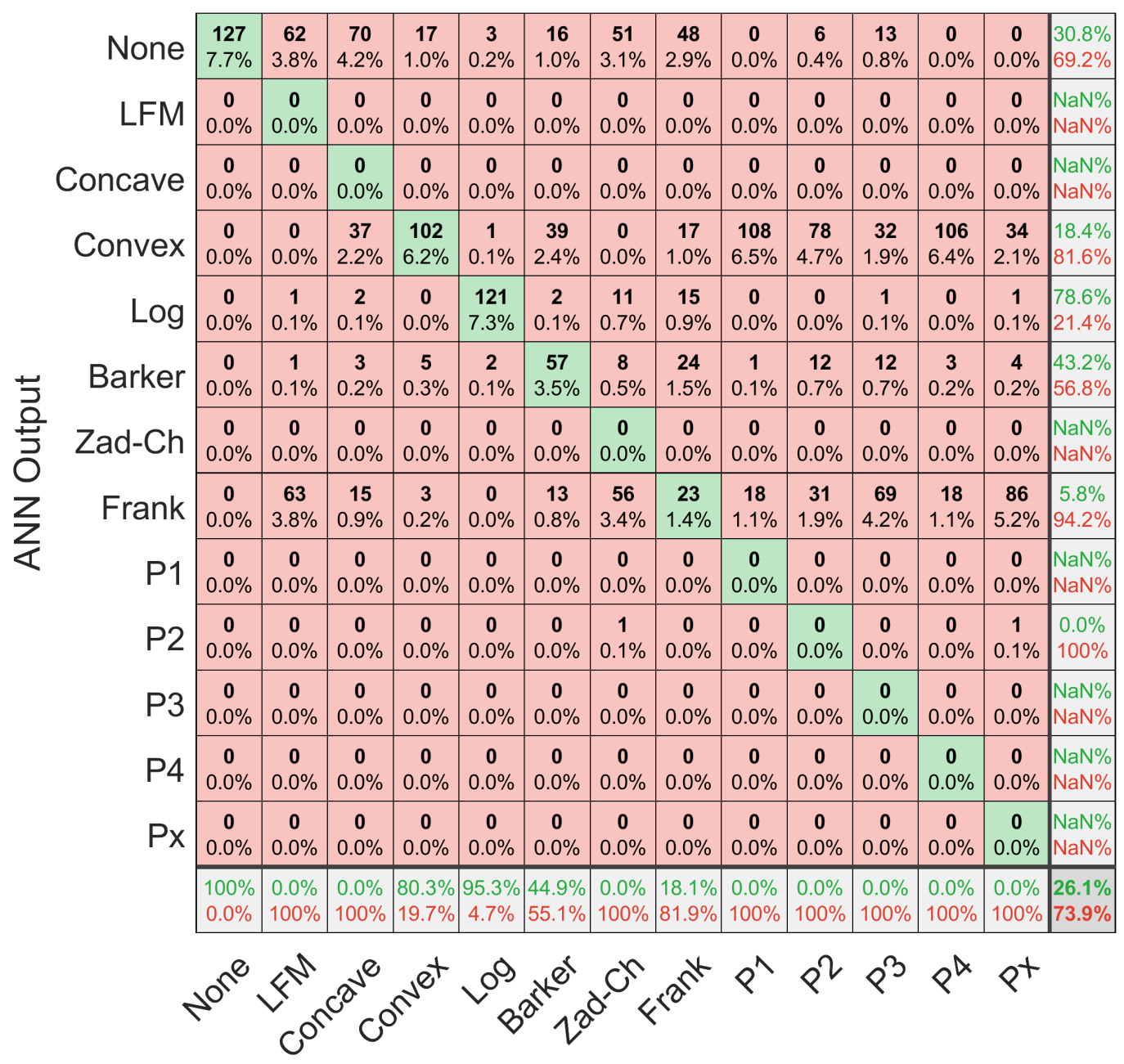

\section{True Class}

Figure 56: CNN Test 2 Confusion Matrix for $-6 \mathrm{~dB}$ 


\section{C.6 CNN Test 3 Confusion Matrices}

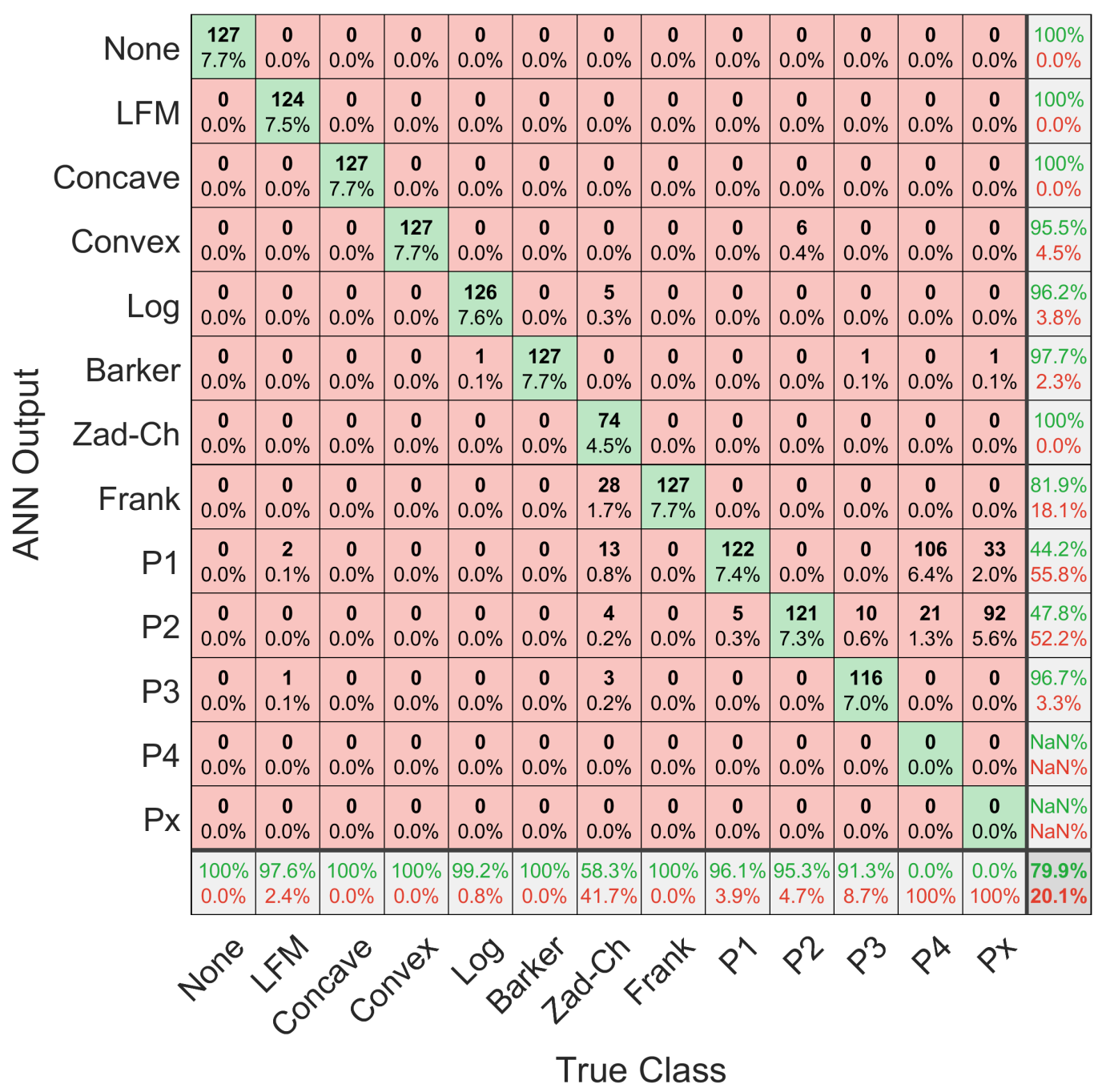

Figure 57: CNN Test 3 Confusion Matrix for $6 \mathrm{~dB}$ 


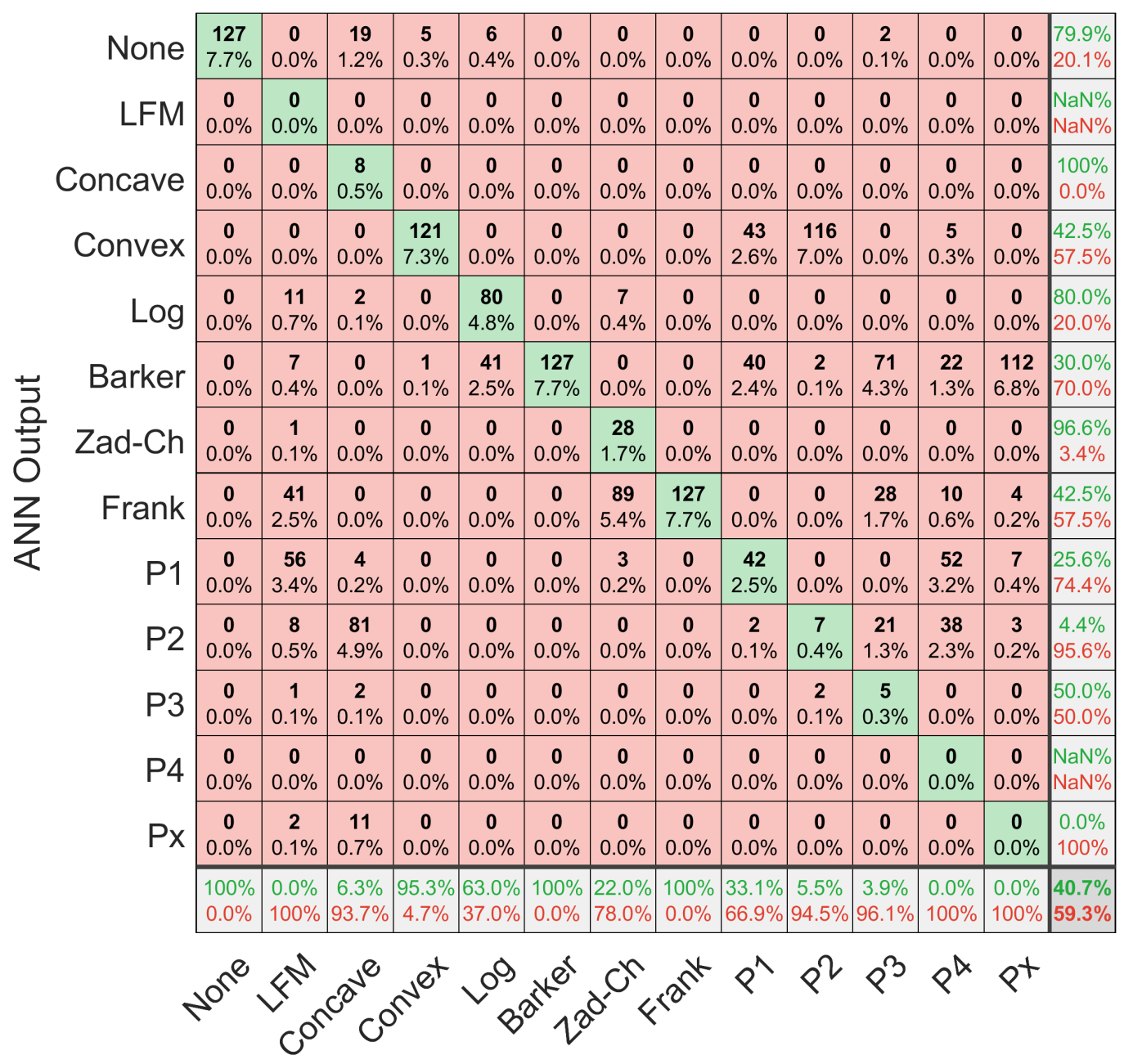

\section{True Class}

Figure 58: CNN Test 3 Confusion Matrix for $0 \mathrm{~dB}$ 


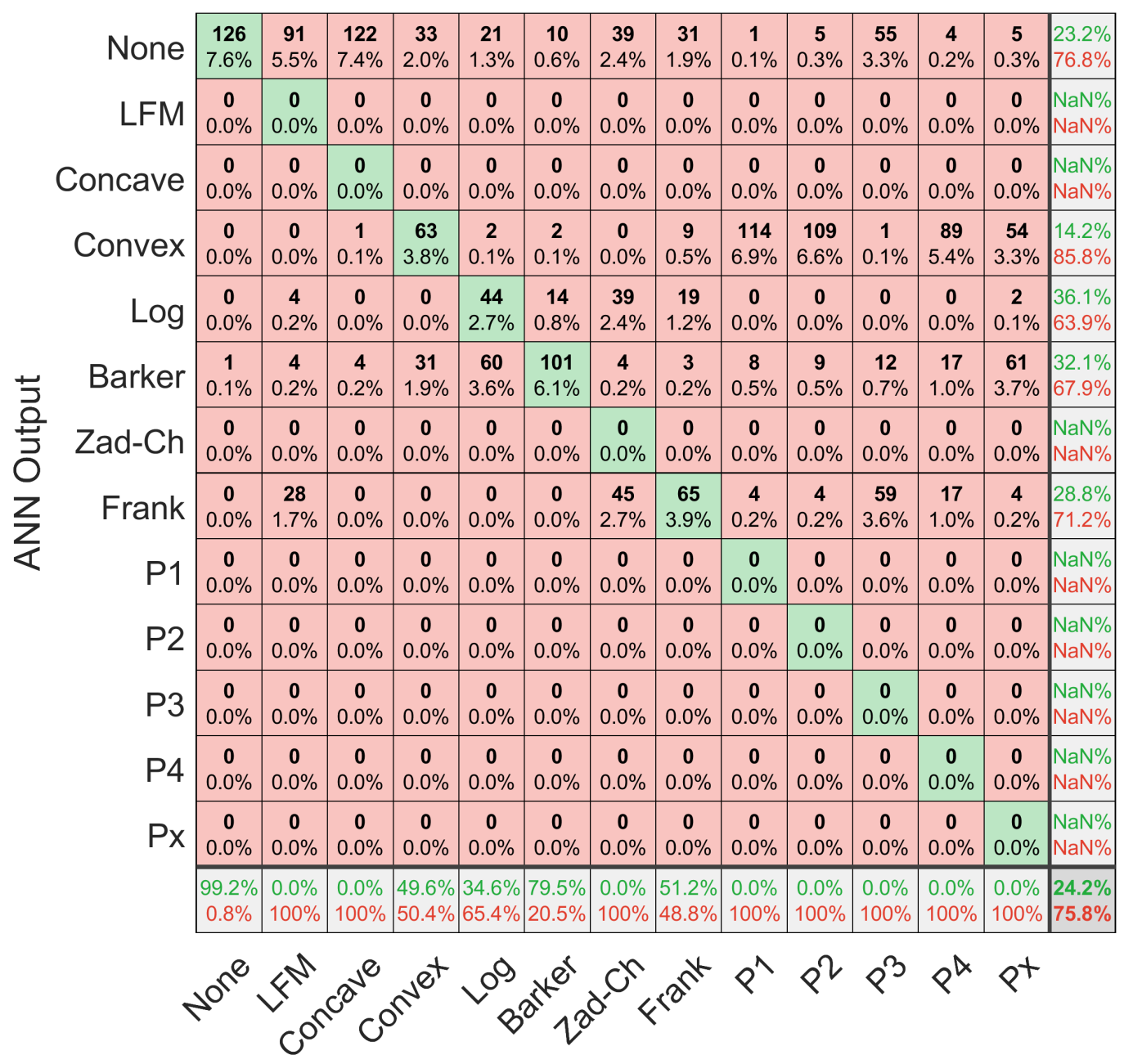

\section{True Class}

Figure 59: CNN Test 3 Confusion Matrix for $-6 \mathrm{~dB}$ 


\section{C.7 Carrier Frequency Confusion Matrices}

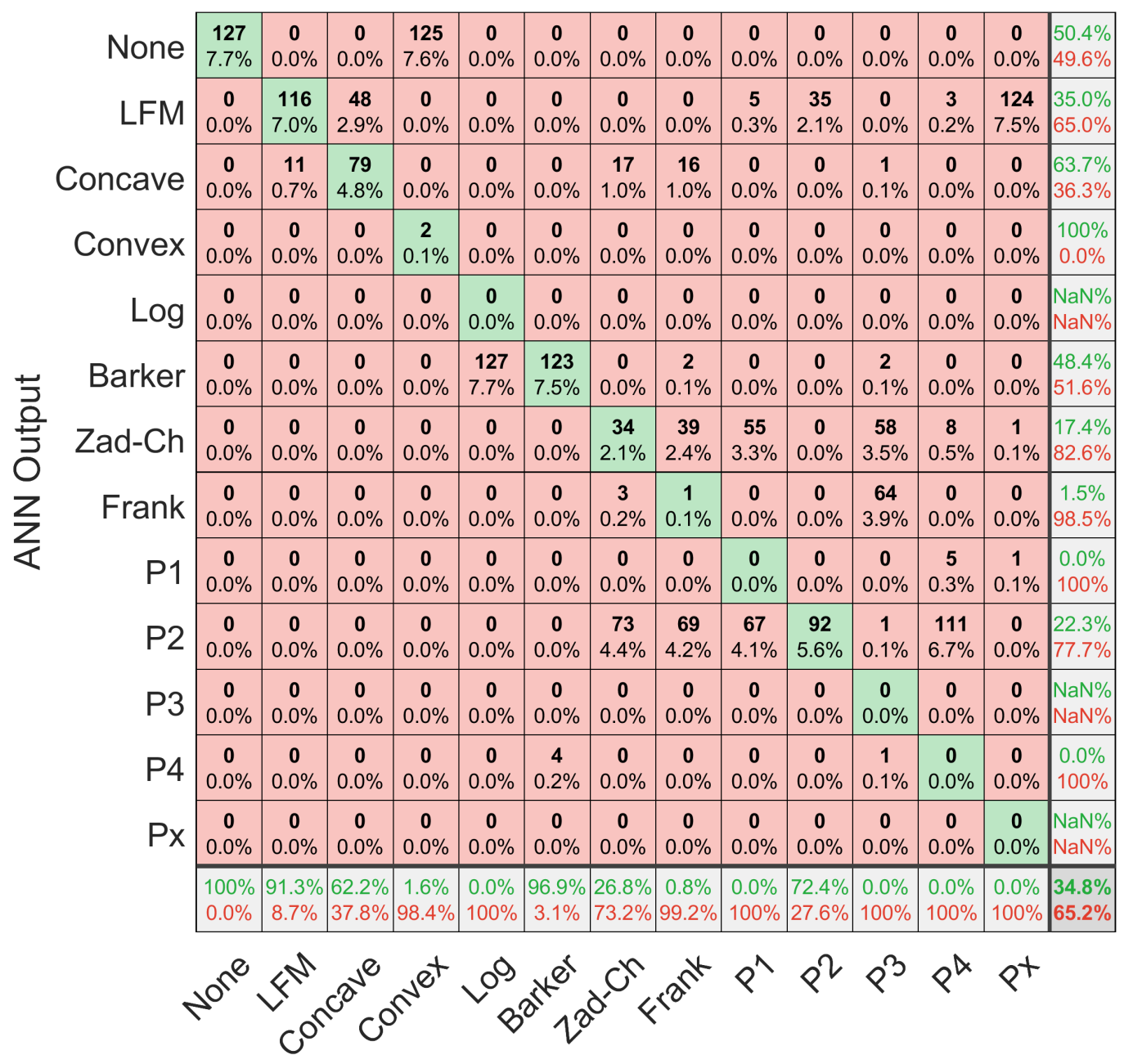

\section{True Class}

Figure 60: ANN Test 4 Confusion Matrix for $6 \mathrm{~dB}$ 




\section{True Class}

Figure 61: ANN Test 5 Confusion Matrix for $6 \mathrm{~dB}$ 


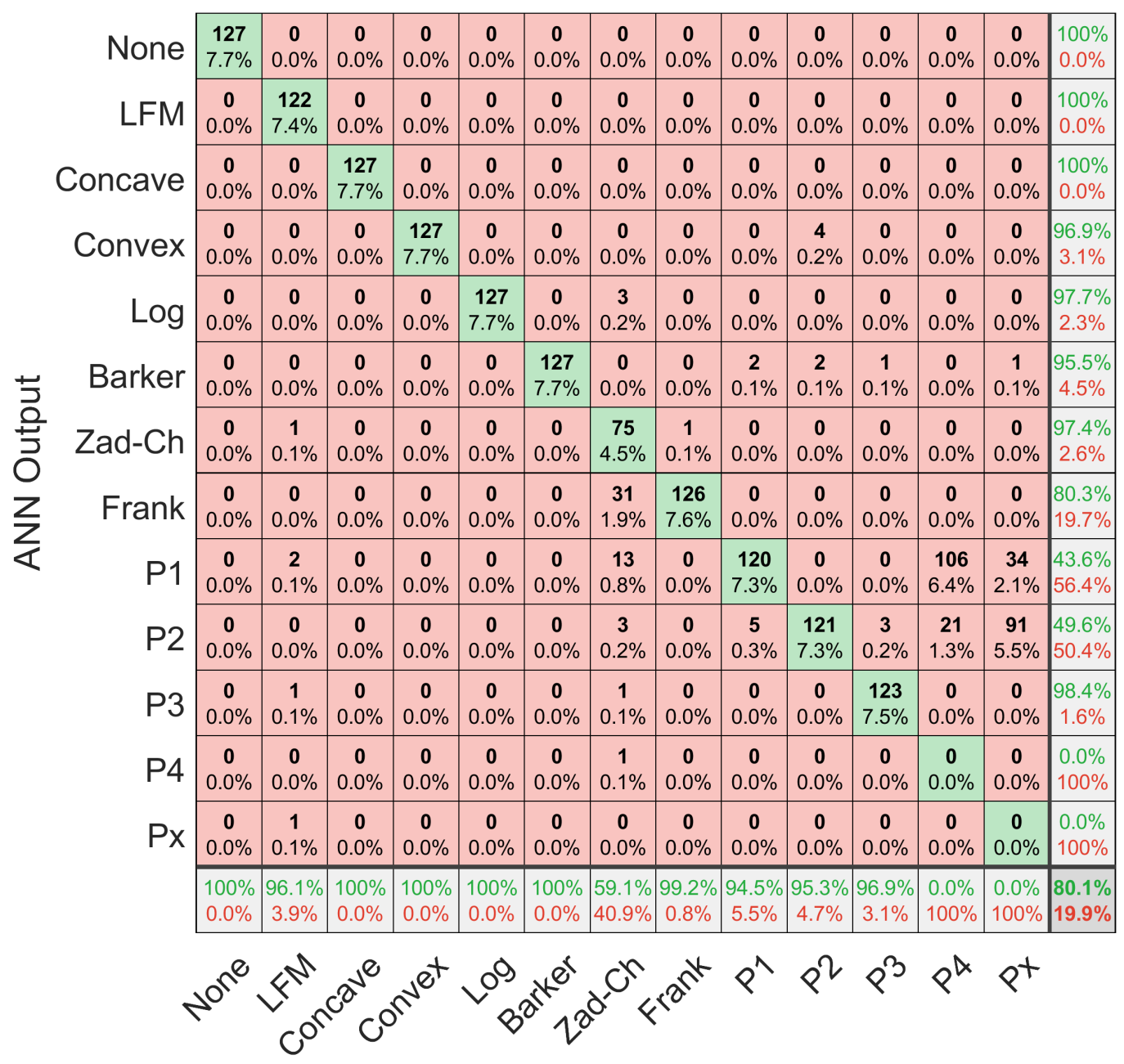

\section{True Class}

Figure 62: CNN Test 4 at Confusion Matrix for $6 \mathrm{~dB}$ 


\begin{tabular}{|c|c|c|c|c|c|c|c|c|c|c|c|c|c|c|}
\hline None & $\begin{array}{c}127 \\
7.7 \% \\
\end{array}$ & $\begin{array}{c}\mathbf{0} \\
0.0 \%\end{array}$ & $\begin{array}{c}\mathbf{0} \\
0.0 \%\end{array}$ & $\begin{array}{c}\mathbf{0} \\
0.0 \%\end{array}$ & $\begin{array}{c}\mathbf{0} \\
0.0 \%\end{array}$ & $\begin{array}{c}\mathbf{0} \\
0.0 \%\end{array}$ & $\begin{array}{c}\mathbf{0} \\
0.0 \%\end{array}$ & $\begin{array}{c}0 \\
0.0 \%\end{array}$ & $\begin{array}{c}\mathbf{0} \\
0.0 \%\end{array}$ & $\begin{array}{c}0 \\
0.0 \%\end{array}$ & $\begin{array}{c}\mathbf{0} \\
0.0 \%\end{array}$ & $\begin{array}{c}\mathbf{0} \\
0.0 \%\end{array}$ & $\begin{array}{c}\mathbf{0} \\
0.0 \%\end{array}$ & $\begin{array}{l}100 \% \\
0.0 \%\end{array}$ \\
\hline LFM & $\begin{array}{c}\mathbf{0} \\
0.0 \%\end{array}$ & $\begin{array}{c}125 \\
7.6 \%\end{array}$ & $\begin{array}{c}\mathbf{0} \\
0.0 \%\end{array}$ & $\begin{array}{c}\mathbf{0} \\
0.0 \%\end{array}$ & $\begin{array}{c}\mathbf{0} \\
0.0 \%\end{array}$ & $\begin{array}{c}\mathbf{0} \\
0.0 \%\end{array}$ & $\begin{array}{c}\mathbf{0} \\
0.0 \%\end{array}$ & $\begin{array}{c}0 \\
0.0 \%\end{array}$ & $\begin{array}{c}\mathbf{0} \\
0.0 \% \\
\end{array}$ & $\begin{array}{c}0 \\
0.0 \%\end{array}$ & $\begin{array}{c}\mathbf{0} \\
0.0 \%\end{array}$ & \begin{tabular}{c|}
$\mathbf{0}$ \\
$0.0 \%$ \\
\end{tabular} & $\begin{array}{c}0 \\
0.0 \%\end{array}$ & $\begin{array}{l}100 \% \\
0.0 \%\end{array}$ \\
\hline Cor & $\begin{array}{c}\mathbf{0} \\
0.0 \% \\
\end{array}$ & $\begin{array}{c}\mathbf{0} \\
0.0 \% \\
\end{array}$ & $\begin{array}{c}127 \\
7.7 \% \\
\end{array}$ & $\begin{array}{c}\mathbf{0} \\
0.0 \% \\
\end{array}$ & $\begin{array}{c}\mathbf{0} \\
0.0 \%\end{array}$ & $\begin{array}{c}\mathbf{0} \\
0.0 \%\end{array}$ & $\begin{array}{c}\mathbf{0} \\
0.0 \%\end{array}$ & $\begin{array}{c}0 \\
0.0 \%\end{array}$ & $\begin{array}{c}\mathbf{0} \\
0.0 \%\end{array}$ & $\begin{array}{c}0 \\
0.0 \% \\
\end{array}$ & $\begin{array}{c}\mathbf{0} \\
0.0 \%\end{array}$ & $\begin{array}{c}\mathbf{0} \\
0.0 \% \\
\end{array}$ & $\begin{array}{c}\mathbf{0} \\
0.0 \%\end{array}$ & $\begin{array}{l}100 \% \\
0.0 \% \\
\end{array}$ \\
\hline Co & $\begin{array}{c}\mathbf{0} \\
0.0 \%\end{array}$ & $\begin{array}{c}0 \\
0.0 \%\end{array}$ & $\begin{array}{c}\mathbf{0} \\
0.0 \%\end{array}$ & \begin{tabular}{c|}
$\mathbf{1 2 7}$ \\
$7.7 \%$ \\
\end{tabular} & $\begin{array}{c}\mathbf{0} \\
0.0 \%\end{array}$ & $\begin{array}{c}\mathbf{0} \\
0.0 \%\end{array}$ & $\begin{array}{c}\mathbf{0} \\
0.0 \%\end{array}$ & $\begin{array}{c}0 \\
0.0 \%\end{array}$ & $\begin{array}{c}\mathbf{0} \\
0.0 \%\end{array}$ & $\begin{array}{c}3 \\
0.2 \%\end{array}$ & $\begin{array}{c}\mathbf{0} \\
0.0 \%\end{array}$ & $\begin{array}{c}\mathbf{0} \\
0.0 \%\end{array}$ & $\begin{array}{c}0 \\
0.0 \%\end{array}$ & \begin{tabular}{|c|}
$97.7 \%$ \\
$2.3 \%$
\end{tabular} \\
\hline LUS & \begin{tabular}{|c|}
$\mathbf{0}$ \\
$0.0 \%$
\end{tabular} & $\begin{array}{c}\mathbf{0} \\
0.0 \%\end{array}$ & $\begin{array}{c}\mathbf{0} \\
0.0 \%\end{array}$ & $\begin{array}{c}\mathbf{0} \\
0.0 \%\end{array}$ & $\begin{array}{c}125 \\
7.6 \%\end{array}$ & $\begin{array}{c}\mathbf{0} \\
0.0 \%\end{array}$ & $\begin{array}{c}1 \\
0.1 \%\end{array}$ & $\begin{array}{c}\mathbf{0} \\
0.0 \%\end{array}$ & $\begin{array}{c}\mathbf{0} \\
0.0 \%\end{array}$ & $\begin{array}{c}0 \\
0.0 \%\end{array}$ & $\begin{array}{c}\mathbf{0} \\
0.0 \%\end{array}$ & \begin{tabular}{c|}
$\mathbf{0}$ \\
$0.0 \%$ \\
\end{tabular} & $\begin{array}{c}\mathbf{0} \\
0.0 \%\end{array}$ & $\begin{array}{c}99.2 \% \\
0.8 \%\end{array}$ \\
\hline Barl & $\begin{array}{c}\mathbf{0} \\
0.0 \%\end{array}$ & $\begin{array}{c}\mathbf{0} \\
0.0 \%\end{array}$ & $\begin{array}{c}\mathbf{0} \\
0.0 \%\end{array}$ & \begin{tabular}{|c|}
$\mathbf{0}$ \\
$0.0 \%$
\end{tabular} & $\begin{array}{c}\mathbf{2} \\
0.1 \%\end{array}$ & \begin{tabular}{|c|}
$\mathbf{1 2 7}$ \\
$7.7 \%$ \\
\end{tabular} & $\begin{array}{c}\mathbf{0} \\
0.0 \%\end{array}$ & $\begin{array}{c}\mathbf{0} \\
0.0 \%\end{array}$ & $\begin{array}{c}\mathbf{2} \\
0.1 \%\end{array}$ & $\begin{array}{c}\mathbf{0} \\
0.0 \%\end{array}$ & $\begin{array}{c}\mathbf{1} \\
0.1 \% \\
\end{array}$ & $\begin{array}{c}\mathbf{0} \\
0.0 \% \\
\end{array}$ & $\begin{array}{c}\mathbf{0} \\
0.0 \%\end{array}$ & \begin{tabular}{|c|}
$96.2 \%$ \\
$3.8 \%$ \\
\end{tabular} \\
\hline Zac & $\begin{array}{c}\mathbf{0} \\
0.0 \% \\
\end{array}$ & $\begin{array}{c}0 \\
0.0 \% \\
\end{array}$ & $\begin{array}{c}\mathbf{0} \\
0.0 \% \\
\end{array}$ & $\begin{array}{c}\mathbf{0} \\
0.0 \% \\
\end{array}$ & $\begin{array}{c}\mathbf{0} \\
0.0 \% \\
\end{array}$ & $\begin{array}{c}\mathbf{0} \\
0.0 \% \\
\end{array}$ & $\begin{array}{c}90 \\
5.5 \%\end{array}$ & $\begin{array}{c}0 \\
0.0 \% \\
\end{array}$ & $\begin{array}{c}0 \\
0.0 \% \\
\end{array}$ & $\begin{array}{c}0 \\
0.0 \% \\
\end{array}$ & $\begin{array}{c}\mathbf{0} \\
0.0 \% \\
\end{array}$ & \begin{tabular}{|c|}
$\mathbf{0}$ \\
$0.0 \%$ \\
\end{tabular} & $\begin{array}{c}\mathbf{0} \\
0.0 \% \\
\end{array}$ & \begin{tabular}{|l|}
$100 \%$ \\
$0.0 \%$ \\
\end{tabular} \\
\hline Fra & $\begin{array}{c}\mathbf{0} \\
0.0 \% \\
\end{array}$ & $\begin{array}{c}\mathbf{0} \\
0.0 \% \\
\end{array}$ & $\begin{array}{c}0 \\
0.0 \% \\
\end{array}$ & $\begin{array}{c}\mathbf{0} \\
0.0 \% \\
\end{array}$ & $\begin{array}{c}\mathbf{0} \\
0.0 \% \\
\end{array}$ & $\begin{array}{c}\mathbf{0} \\
0.0 \%\end{array}$ & $\begin{array}{c}\mathbf{2 1} \\
1.3 \% \\
\end{array}$ & $\begin{array}{c}127 \\
7.7 \% \\
\end{array}$ & $\begin{array}{c}\mathbf{0} \\
0.0 \% \\
\end{array}$ & $\begin{array}{c}0 \\
0.0 \% \\
\end{array}$ & $\begin{array}{c}0 \\
0.0 \%\end{array}$ & $\begin{array}{c}\mathbf{0} \\
0.0 \%\end{array}$ & $\begin{array}{c}\mathbf{0} \\
0.0 \%\end{array}$ & \begin{tabular}{|l|}
$85.8 \%$ \\
$14.2 \%$ \\
\end{tabular} \\
\hline & $\begin{array}{c}\mathbf{0} \\
0.0 \%\end{array}$ & $\begin{array}{c}1 \\
0.1 \% \\
\end{array}$ & $\begin{array}{c}0 \\
0.0 \% \\
\end{array}$ & $\begin{array}{c}\mathbf{0} \\
0.0 \%\end{array}$ & $\begin{array}{c}\mathbf{0} \\
0.0 \% \\
\end{array}$ & $\begin{array}{c}\mathbf{0} \\
0.0 \%\end{array}$ & $\begin{array}{c}8 \\
0.5 \% \\
\end{array}$ & $\begin{array}{c}0 \\
0.0 \%\end{array}$ & $\begin{array}{c}115 \\
7.0 \% \\
\end{array}$ & $\begin{array}{c}0 \\
0.0 \% \\
\end{array}$ & $\begin{array}{c}\mathbf{0} \\
0.0 \% \\
\end{array}$ & $\begin{array}{c}\mathbf{1 0 7} \\
6.5 \% \\
\end{array}$ & $\begin{array}{c}36 \\
2.2 \% \\
\end{array}$ & \begin{tabular}{|l|}
$43.1 \%$ \\
$56.9 \%$ \\
\end{tabular} \\
\hline $\mathrm{P} 2$ & $\begin{array}{c}\mathbf{0} \\
0.0 \%\end{array}$ & $\begin{array}{c}\mathbf{0} \\
0.0 \%\end{array}$ & $\begin{array}{c}\mathbf{0} \\
0.0 \% \\
\end{array}$ & $\begin{array}{c}\mathbf{0} \\
0.0 \%\end{array}$ & $\begin{array}{c}\mathbf{0} \\
0.0 \%\end{array}$ & $\begin{array}{c}\mathbf{0} \\
0.0 \%\end{array}$ & $\begin{array}{c}\mathbf{2} \\
0.1 \%\end{array}$ & $\begin{array}{c}0 \\
0.0 \%\end{array}$ & $\begin{array}{c}10 \\
0.6 \%\end{array}$ & $\begin{array}{c}123 \\
7.5 \% \\
\end{array}$ & $\begin{array}{c}10 \\
0.6 \%\end{array}$ & \begin{tabular}{|c|}
$\mathbf{2 0}$ \\
$1.2 \%$ \\
\end{tabular} & $\begin{array}{c}\mathbf{9 0} \\
5.5 \%\end{array}$ & \begin{tabular}{|l|}
$48.2 \%$ \\
$51.8 \%$ \\
\end{tabular} \\
\hline$P 3$ & $\begin{array}{c}\mathbf{0} \\
0.0 \%\end{array}$ & $\begin{array}{c}0 \\
0.0 \%\end{array}$ & $\begin{array}{c}\mathbf{0} \\
0.0 \%\end{array}$ & $\begin{array}{c}\mathbf{0} \\
0.0 \%\end{array}$ & $\begin{array}{c}\mathbf{0} \\
0.0 \%\end{array}$ & $\begin{array}{c}\mathbf{0} \\
0.0 \%\end{array}$ & $\begin{array}{c}\mathbf{5} \\
0.3 \%\end{array}$ & $\begin{array}{c}0 \\
0.0 \%\end{array}$ & $\begin{array}{c}0 \\
0.0 \%\end{array}$ & $\begin{array}{c}1 \\
0.1 \%\end{array}$ & $\begin{array}{c}116 \\
7.0 \%\end{array}$ & $\begin{array}{c}\mathbf{0} \\
0.0 \%\end{array}$ & $\begin{array}{c}0 \\
0.0 \%\end{array}$ & $\begin{array}{c}95.1 \% \\
4.9 \%\end{array}$ \\
\hline P4 & $\begin{array}{c}\mathbf{0} \\
0.0 \% \\
\end{array}$ & $\begin{array}{c}\mathbf{0} \\
0.0 \%\end{array}$ & $\begin{array}{c}\mathbf{0} \\
0.0 \% \\
\end{array}$ & $\begin{array}{c}\mathbf{0} \\
0.0 \% \\
\end{array}$ & $\begin{array}{c}\mathbf{0} \\
0.0 \%\end{array}$ & $\begin{array}{c}\mathbf{0} \\
0.0 \%\end{array}$ & $\begin{array}{c}\mathbf{0} \\
0.0 \%\end{array}$ & $\begin{array}{c}0 \\
0.0 \%\end{array}$ & $\begin{array}{c}\mathbf{0} \\
0.0 \%\end{array}$ & $\begin{array}{c}0 \\
0.0 \% \\
\end{array}$ & $\begin{array}{c}\mathbf{0} \\
0.0 \% \\
\end{array}$ & \begin{tabular}{|c|}
$\mathbf{0}$ \\
$0.0 \%$
\end{tabular} & $\begin{array}{c}\mathbf{0} \\
0.0 \% \\
\end{array}$ & $\begin{array}{l}\text { NaN\% } \\
\text { NaN\% } \\
\end{array}$ \\
\hline \multirow[t]{2}{*}{$P_{x}$} & $\begin{array}{c}\mathbf{0} \\
0.0 \%\end{array}$ & $\begin{array}{c}1 \\
0.1 \%\end{array}$ & $\begin{array}{c}\mathbf{0} \\
0.0 \%\end{array}$ & $\begin{array}{c}\mathbf{0} \\
0.0 \%\end{array}$ & $\begin{array}{c}\mathbf{0} \\
0.0 \%\end{array}$ & $\begin{array}{c}\mathbf{0} \\
0.0 \%\end{array}$ & $\begin{array}{c}\mathbf{0} \\
0.0 \%\end{array}$ & $\begin{array}{c}0 \\
0.0 \%\end{array}$ & $\begin{array}{c}\mathbf{0} \\
0.0 \%\end{array}$ & $\begin{array}{c}\mathbf{0} \\
0.0 \%\end{array}$ & $\begin{array}{c}\mathbf{0} \\
0.0 \%\end{array}$ & $\begin{array}{c}\mathbf{0} \\
0.0 \%\end{array}$ & $\begin{array}{c}0 \\
0.0 \%\end{array}$ & $\begin{array}{l}0.0 \% \\
100 \%\end{array}$ \\
\hline & $\begin{array}{l}100 \% \\
0.0 \%\end{array}$ & $\begin{array}{l}98.4 \% \\
1.6 \%\end{array}$ & $\begin{array}{l}100 \% \\
0.0 \% \\
\end{array}$ & $\begin{array}{l}100 \% \\
0.0 \% \\
\end{array}$ & $\begin{array}{c}98.4 \% \\
1.6 \% \\
\end{array}$ & $\begin{array}{l}100 \% \\
0.0 \% \\
\end{array}$ & \begin{tabular}{|l|}
$70.9 \%$ \\
$29.1 \%$
\end{tabular} & $\begin{array}{l}100 \% \\
0.0 \% \\
\end{array}$ & \begin{tabular}{|c|}
$90.6 \%$ \\
$9.4 \%$ \\
\end{tabular} & $\begin{array}{c}96.9 \% \\
3.1 \%\end{array}$ & $\begin{array}{c}91.3 \% \\
8.7 \%\end{array}$ & $\begin{array}{l}0.0 \% \\
100 \% \\
\end{array}$ & \begin{tabular}{|l|}
$0.0 \%$ \\
$100 \%$ \\
\end{tabular} & \begin{tabular}{|l|}
$80.5 \%$ \\
$19.5 \%$ \\
\end{tabular} \\
\hline
\end{tabular}

\section{True Class}

Figure 63: CNN Test 5 Confusion Matrix for $6 \mathrm{~dB}$ 